\title{
A Multi-Criteria Decision Framework for Unstructured Complex Problem: A Strategy for Biofuel Production
}

\author{
by \\ Mostafa F. Fawzy \\ A dissertation submitted to the graduate faculty \\ DOCTOR OF PHILOSOPHY \\ Major: Industrial Engineering \\ Program of Study Committee: \\ Paul J. Componation, Major Professor \\ Guiping Hu, Co-Major Professor \\ Caroline Krejci \\ Iris Rivero \\ Mark Mba Wright \\ Robert C. Brown \\ Iowa State University \\ Ames, Iowa \\ 2015
} in partial fulfillment of the requirements for the degree of

Copyright ( ) Mostafa F. Fawzy, 2015. All rights reserved. 


\section{DEDICATION}

This dissertation is dedicated to those who have been on my side and have given me support and encouragement: my parents, grandmother, wife, brother, sister, brother-in-law, nephews, father and mother-in-law, my wife's sisters and brother, and my friends.

I would like to mention that it has been a great sacrifice for me to work on a real life

problem, but all the suffering and pressure have been worth it because I have been trained not only in theoretical knowledge, which is a good base for the future, but also in facing difficulties, achieving self-confidence, and realizing the results of my efforts. 
TABLE OF CONTENTS

NOMENCLATURE

ACKNOWLEDGEMENTS _....................................................................... viii

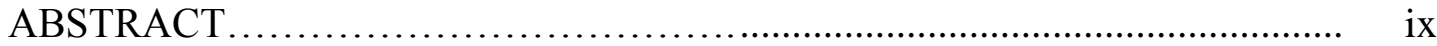

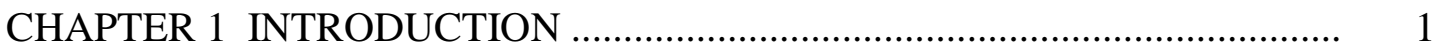

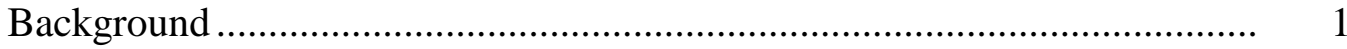

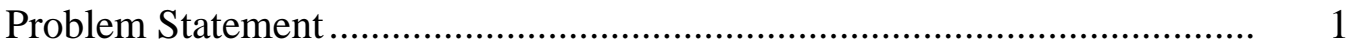

Dissertation Structure ...............................................................................

CHAPTER 2 BIOFUEL PRODUCTION: FAST PYROLYSIS UNITS'

MANUFACTURING INFRASTRUCTURE ....................................................... 6

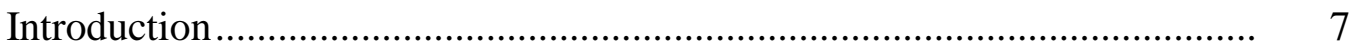

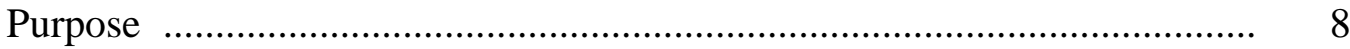

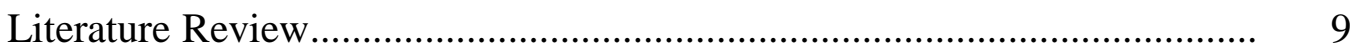

The Methodology and Proposed Approach ……………............................. 17

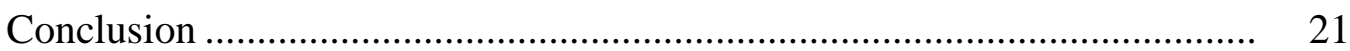

CHAPTER 3 BIOFUEL PRODUCTION: STAKEHOLDERS'

IDENTIFICATION .................................................................................. 23

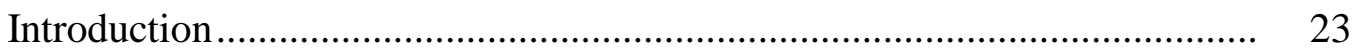

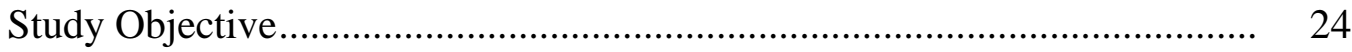

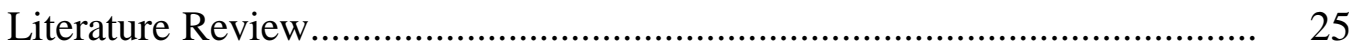

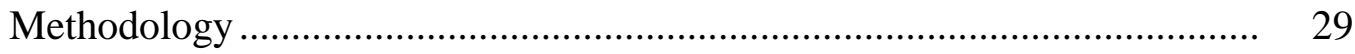

BPS Identification and Analysis ............................................................... 29 
Result and Discussion .......................................................................... 31

Assumptions and limitations................................................................. 34

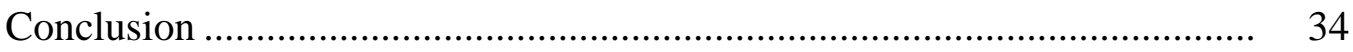

Recommendations and Future Work ………………................................. 35

CHAPTER 4 BIOFUEL PRODUCTION: UTILIZING STAKEHOLDERS'

PERSPECTIVES............................................................................... 36

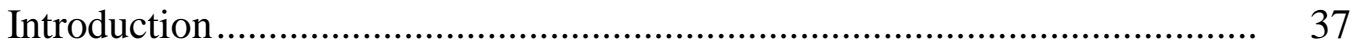

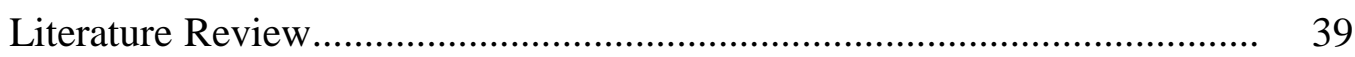

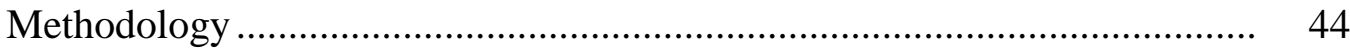

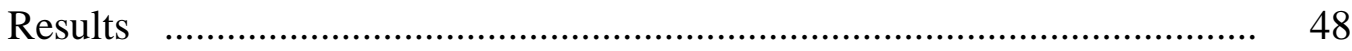

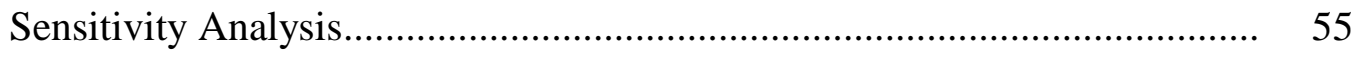

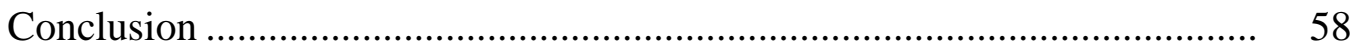

CHAPTER 5 STAKEHOLDERS' REQUIREMENTS ASSESSMENT FOR BIOFUEL PRODUCTION ..................................................................... 62

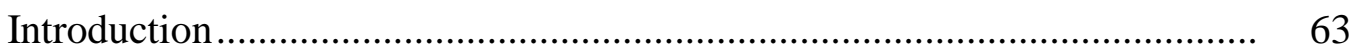

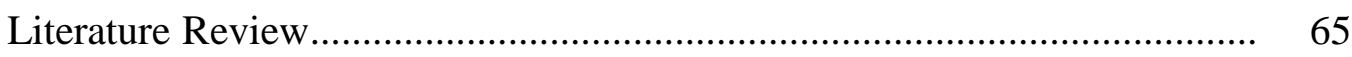

Methodology …….............................................................................

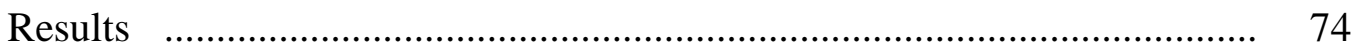

Sensitivity Analysis.............................................................................. 78

Conclusion and Recommendations ........................................................... 84

CHAPTER 6 A MULTI-CRITERIA DECISION FRAMEWORK FOR AN UNSTRUCTURED COMPLEX PROBLEM: BIOFUEL UNIT

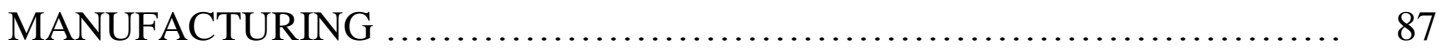

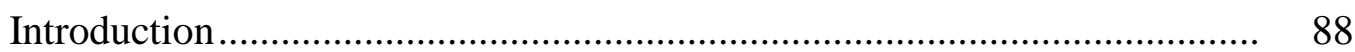

Literature Review............................................................................. 90 


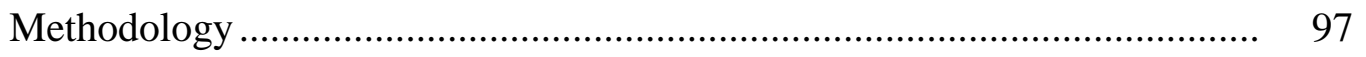

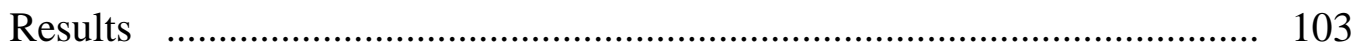

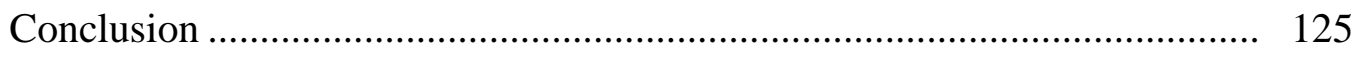

CHAPTER 7 GENERAL CONCLUSIONS ................................................... 129

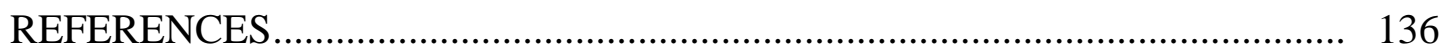




\section{NOMENCLATURE}

AHP

AMT

$\mathrm{BM}$

bpd

BPS

BPM

CBA

DEA

DM

EBTP

ELECTRE

GHG

FPU

MAUT

MCDA

MCDM

MODM

RFS

SMART

SME

SME
Analytic Hierarchy Process

Advance Manufacturing Technology

Biofuel Manufacturing

Barrels Per Day

Biofuel Production Stakeholders

Biomass Processing Modules

Cost Benefit Analysis

Data Envelope Analysis

Decision-Making

European Biofuels Technology Platform

ELimination and Choice Expressing REality

Greenhouse Gas

Fast Pyrolysis Unit

Multi-Attribute Utility Theory

Multi-Criteria Decision Analysis

Multi-Criteria Decision-Making

Multi-Objective Decision Making

Renewable Fuel Standard

Simple Multi-Attribute Rating Technique

Subject Matter Experts

Small to Medium sized Enterprises 
TOPSIS

tpd

TVP

WSM
Technique for Order of Preference by Similarity to Ideal Solution

Tons Per Day

Technology Value Pyramid

Weighting Sum Method 


\section{ACKNOWLEDGEMENTS}

First, I thank almighty Allah Who gave me the strength to finish this dissertation in an appropriate way and granted me His numerous blessings to achieve this project's objectives.

I then thank everyone who has supported me throughout my dissertation whether physically or emotionally. I especially thank my parents and grandmother who have continuously supported me emotionally throughout the years of my study, including the writing of this dissertation. Also, I love to thank my beloved wife who has supported me in all possible aspects in the writing of this dissertation and who passed hours of patience, respect and love.

I would like to thank my committee chair and advisor, Prof. Paul J. Componation, who taught me more than anybody else throughout the course of this research; a great teacher and mentor. I then thank my co-advisor, Dr. Guiping $\mathrm{Hu}$, who has supported and guided me throughout this study.

In addition, I would also like to thank my committee members, Prof. Robert Brown, Dr. Caroline Krejci, Dr. Mark Mba-Wright, and Dr. Iris Rivero for all their advices and help through my study. I then thank my research sponsors, friends, colleagues, the department faculty and staff for making my time at Iowa State University a wonderful experience. I want

to also offer my appreciation to those who were willing to participate in my interviews and data collection, without whom, this dissertation would not have been possible.

Finally, thanks to my sister, brother and all family members for their continuous support and encouragement. 


\begin{abstract}
A Multi-Criteria Decision Analysis (MCDA) approach was designed and used to evaluate different Fast Pyrolysis Unit (FPU) sizes. The MCDA approach is implemented via two models: Excel worksheet and automated model via Logical Decision ${ }^{\circledR}$ software. The proposed MCDA approach is an integration of the Pugh Concept Selection Matrix, Weighting Sum Method (WSM), and sensitivity analysis using Logical Decision® software. The data for the problem was collected from ten Subject Matter Experts (SMEs) using Pugh Matrix. In addition, two other integrated MCDA approaches were used to solve the same problem. The first approach integrated the Pugh Matrix and WSM. The second approach integrated the Pugh Matrix and the Technique for Order of Preference by Similarity to Ideal Solution (TOPSIS). The designed framework is presented to identify Biofuel Production Stakeholders (BPS), their perspectives, and their requirements. The small FPU was found to be the best alternative using the three approaches. Furthermore, all these approaches allowed ranking of different alternatives based on the five perspectives of manufacturing biofuel production units: economic, environmental, technical, legal, and social perspectives. These five perspectives rely on 18 requirements that were frequently mentioned in previous research. The use of each approach gave different insight about the problem which could help decision-makers to understand the problem better and discuss the alternatives in depth. Sensitivity analysis suggested that the medium FPU is the best alternative in specific conditions under the perspectives-level analysis. On the other hand, it was suggested that the large FPU is the best alternative under specific conditions at the requirements-level analysis. An interesting finding from this research is that from the environmental perspective the
\end{abstract}


medium FPU is recommended as the best alternative instead of the small FPU. In addition, the TOPSIS analysis provided the theoretical positive and negative ideal solutions to help the decision makers gain a better perception of the optimal design of FPUs. Moreover, WSM was found to be the simplest MCDA tool to use. In contrast, TOPSIS was found to be a more complicated tool yet similar to WSM both could not examine result robustness. The proposed approach provided the result robustness limitations. 


\section{CHAPTER 1. INTRODUCTION}

\section{Background}

The use of renewable energy has drawn the attention of many countries because it is viewed as a secure and sustainable replacement for traditional energy sources. Renewable energy is seen as potentially lowering costs, reducing pollution, and improving energy production sustainability. Recent technological advances have resulted in renewable energy being used in multiple applications such as transportation, residential, commerce, and electric power (EIA, 2014).

The United States has been increasing its focus on renewable energy since the energy crisis in the 1970s. Solar, wind, geothermal hydropower (hydroelectricity), hydrokinetic and biofuel energy have been explored. One of the challenges the United States, as well as other countries, face is the range of stakeholders that must be satisfied to make renewable energy viable. Stakeholders' interest in economic, environmental, technical, social, and legal aspects need to be considered when making decisions related to selection and development of these energy sources. The range of stakeholders, multiple and often conflicting requirements, limited data, and high levels of uncertainty, make selection of an appropriate renewable energy production strategy a complex problem.

\section{Problem Statement}

There are different decision-making approaches that can be used to address this problem. Unfortunately, decision makers are often skilled in a single approach and apply it for all 
problems they face (Componation, Dorneich, Hu, \& Nicholls, 2013). Each decision-making approach can give different results and not all approaches are appropriate for all problems (Guitouni \& Martel, 1998). Selecting a suitable approach is often a critical step in solving a complex decision making problem. The selected approach for a problem should have the following criteria: (a) "measures to deal with uncertainty," (b) "user friendliness and flexibility," (c) "transparency and communication," and (d) "multi-stakeholder inclusion" (Kurka, 2013).

Decision-making approaches can be a powerful tool for renewable energy development (Wang, Jing, Zhang, \& Zhao, 2009) and in particular for the biofuel production. Biofuel has shown itself to be a viable energy source and approximately half of the renewable energy production in the U.S. is from biomass (EIA, 2014).

Deciding on an appropriate biofuel production strategy meets the criteria for a complex problem. In particular developing an understanding of stakeholder requirement is a significant issue. Researchers have mentioned that the optimal size of a biofuel unit depends on many variables, some unique to this industry such as the issue of transportation costs (Wright \& Brown, 2007; Larasati, Liu, \& Epplin, 2012).

This research will take a systems view of biofuel production and look at the full range of stakeholders' requirements, including economic, environmental, technical, social, and legal perspectives. Specifically, this research will answer the following questions: (1) Which decisionmaking approaches are most commonly applied in this field? (2) Which evaluation criteria (perspectives \& requirements) are appropriate for this industry? (3) Can a better understanding of the solution space for the biofuel production problem be gained by using multiple decisionmaking approaches? (4) Given our current understanding of stakeholders' requirements, which biofuel production strategy is most appropriate? Several decision-making approaches are utilized 
in this effort, including Pugh Concept Selection Matrix, Weighting Sum Method (WSM), and Technique for Order of Preference by Similarity to Ideal Solution (TOPSIS).

\section{Dissertation Structure}

This dissertation is written using the journal paper format. It contains seven chapters. The first chapter (Chapter 1) provides an overview of the dissertation and its structure. Chapters 2 through 6 are publications that address the research questions. These five chapters include manuscripts published in, accepted by, or submitted to scholarly journals and proceedings. The publication status of each manuscript is indicated after each chapter title. The last chapter (Chapter 7) is a general conclusion that is summarizing the results and future work.

Chapter 1 is a general introduction of the dissertation. It introduces the problem, and provides context for the remaining chapters. In addition, this chapter illustrates the document structure with a brief description about each chapter in this dissertation.

Chapter 2 is a paper presented at Fifth International Conference on Industrial Engineering and Operations Management (IEOM 2015), Dubai, United Arab Emirates in March 3, 2015. This paper titled Biofuel production: Fast Pyrolysis Units' Manufacturing Infrastructure. This paper illustrates the general framework and the strategic design for the development of the systems-oriented approach to reduce capital costs, investment risk, and increase units' flexibility to be more responsive to changes in energy demands. This designed decision-making approach in this work could be applied to other complex systems under uncertainty conditions. This paper consider as the foundation of the research and it contributes in answering the fourth research question.

Chapter 3 is a paper published at Journal of Management and Engineering Integration, volume 7, issue 1. This paper titled Biofuel Production: Stakeholders' Identification. This paper 
focuses on identifying the Biofuel Production Stakeholders (BPS). This work is done through a management theory implementation on BPS to confirm all decision-makers' contribution in the process. By the application of modified theory of stakeholder identification and salience, stakeholders, in this research, are evaluated and classified into different groups according to their typologies. This paper contributes in answering the third and fourth research questions. In addition, it considers as the base of the next three papers.

Chapter 4 is a paper published at the Engineering Management Journal, volume 27, issue 2. This paper titled Biofuel Production: Utilizing Stakeholders' Perspectives. This paper focuses on identifying all BPS perspectives and requirements. It includes a comprehensive analysis of all stakeholders' perspectives. Individual subject matter experts reviewed and prioritized a set of requirements that reflected different stakeholders' perspectives, including economic, environmental, technical, social, and legal. The perspectives were then used to analyze multiple fast pyrolysis units to determine which size was the most effective in meeting the perspectives in total. This paper contributes in answering the second, third, and fourth research questions. Also, it paves the way for next chapter.

Chapter 5 is a paper under review for publication at Energy Journal. This paper titled Stakeholders' Requirements Assessment for Biofuel Production. This paper develops a comprehensive study of stakeholders' requirements. Then, identifying the optimal size for a Fast Pyrolysis Unit (FPU) based on individual subject matter experts. These subject matter experts reviewed, ranked, and evaluated the set of requirements for a unity of FPU sizes. This paper contributes in answering the second, third, and fourth research questions.

Chapter 6 is a paper submitted for publication at Applied Energy Journal. This paper titled A Multi-Criteria Decision Framework for an Unstructured Complex Problem: Biofuel Unit 
Manufacturing. This paper investigates the capability of existing decision-making tools to assess the FPU production problem. It also proposes a framework to assess and measure the three different sizes of FPUs for biofuel production, using Pugh Concept Selection matrix with the integration with other decision-making approaches. This research presents the integration between Pugh Concept Selection matrix, WSM, and additional sensitivity analysis as one integrated approach. Moreover, it presents the integration between Pugh Concept Selection matrix, TOPSIS and additional sensitivity analysis as another integrated approach for solving the unstructured complex problem. This paper answering the first research question and contributes in answering the fourth question.

Chapter 7 presents general conclusion summarizes the research results and relates the findings of the five papers. In addition, this chapter includes a general discussion of the result and the recommendations for future work on the research. 


\title{
CHAPTER 2. BIOFUEL PRODUCTION: FAST PYROLYSIS UUNITS' MANUFACTURING INFRASTRUCTURE
}

\author{
A paper published in 2015 International Conference on Industrial Engineering and \\ Operations Management
}

Mostafa F. Fawzy and Paul J. Componation

\begin{abstract}
Biofuels are a replacement for fossil fuels that helps meet national, energy, environmental and economic security requirements. One type of the biofuel production is fast pyrolysis. The goal of this work is to develop an understanding of how fast pyrolysis units can be correctly sized to meet these security types in addition to other stakeholder perspectives and requirements. There are a lot of work in biofuel production; however, most of them are focused on a subset of these perspectives and requirements. Likewise, manufacturing requirements were also not typically studied. There has been some work investigating different sizes of fast pyrolysis units. The manufacturing of fast pyrolysis units is considered as a complex problem due to the stakeholders' diversity as well as the nature of this problem. The diversity of biofuel production stakeholders created different perspectives, requirements, and decisions. Therefore, the need arises to develop a systems-oriented approach for the manufacturing, placement, and optimal sizing of a fast pyrolysis units' manufacturing infrastructure for biofuel energy production. This research aims to illustrate the general framework and the strategic design for the development of
\end{abstract}


the systems-oriented approach to reduce capital costs, investment risk, and increase units' flexibility to be more responsive to changes in energy demands. This designed decision-making approach in this work could be applied to other complex systems for decision-making under uncertainty.

\section{Introduction}

Since 1973, the U.S. realized the importance of using mass for energy production. Then, in 2013, President Obama's State of the Union address identified clean energy technologies as important pillar for future economic development in the U.S. this clean energy is needed to the use in transportation, industries, resident and commerce, and electric power consumptions (EIA, 2014). Recently, biomass is approximately half of the U.S renewable energy production (EIA, 2014).

Biofuel or bio-oil is the form of biomass that is used mainly for transportation (Bridgwater \& Peacocke, 2000), but it could be used for other purposes as well (EIA, 2014). Unlike other renewables, biomass is the only source of solid, liquid, and gaseous fuels (Bridgwater \& Peacocke, 2000). Figure 2.1 demonstrates a summary of thermochemical biomass processes and products as presented in Bridgwater and Peacocke's research (Bridgwater \& Peacocke, 2000).

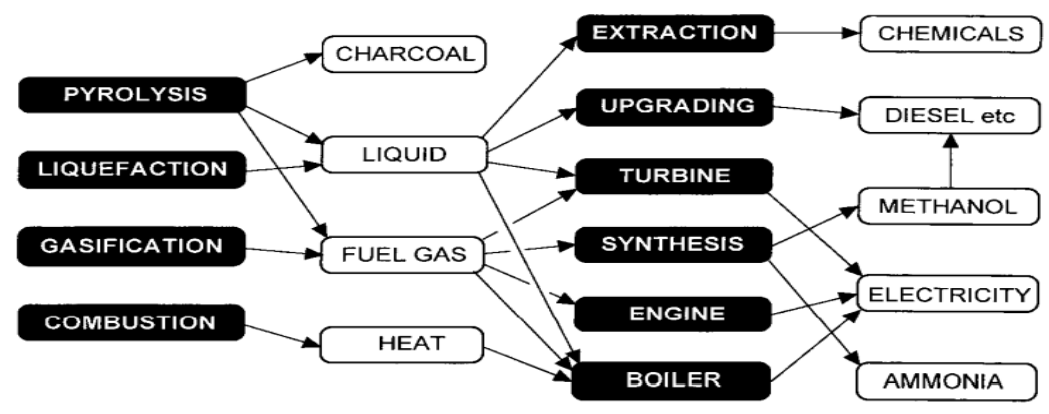

Figure 2.1. Thermochemical biomass processes and products (Bridgwater \& Peacocke, 2000) 
Fast pyrolysis is a thermochemical process in which biomass or any other carbonaceous material is converted into char, bio-oil and non-condensable gases. The manufacturing of the Fast Pyrolysis Unit (FPU) has been studied previously under limited scope (Larasati, Liu, \& Epplin, 2012). Three different FPUs' sizes were investigated. An FPU consumes 2,000 Tons Per Day (tpd) of biomass is studied as a large unit size (Wright, Brown, \& Boateng, 2008). Other FPU that consumes between 200 and 500 tpd is considered as second alternative (Wright, Brown, \& Boateng, 2008; Wright, Daugaard, Satria, \& Brown, 2010). The third proposed FPU is the one that consumes $50 \mathrm{tpd}$, which is considered as a mobile unit (LaClaire, Barrett, \& Hall, 2004).

Due to the different stakeholders' perspectives and requirements in addition to the four different security types, the optimal sizing of FPUs can be classified as a complex decision problem that should be solved using a Multi-Attribute Utility Theory (MAUT) approach (Min, 1994). The MAUT follows the same pattern as other decision-making approaches (Dyer, 2005). Although each decision-making approach uses different terminologies to name the process stages, the essence of these stages are the same (DCLG, 2009; Chelst, \& Canbolat, 2011). All decision-making approaches should have objective identification, two or more alternatives identification, modeling, analyzing, and sensitivity analysis.

\section{Purpose}

The goal of this research is to develop a framework for a systems-oriented approach to the manufacturing, placement, and optimal sizing of an FPUs manufacturing infrastructure that reduces capital costs, investment risk, and is more responsive to changes in energy demands. The proposed approach supports the development of a distributed bioenergy system. This work focuses on part of the manufacturing strategy assessment of biofuel production units (Task 2 and 
part of Task 3) as shown in Figure 2.2. The research output is incorporation into ongoing efforts to further development of bioenergy systems.

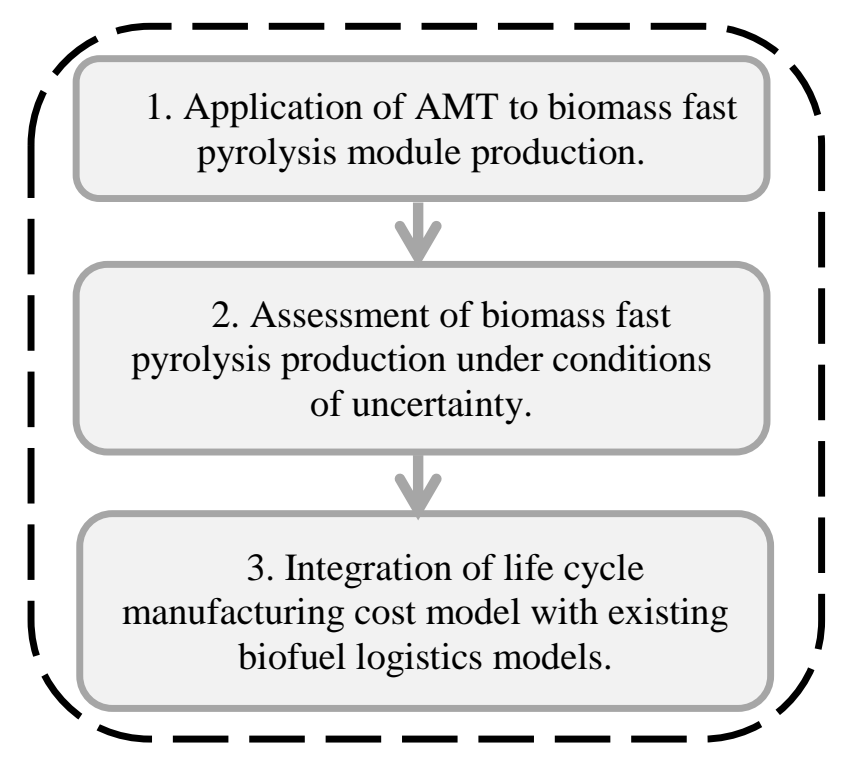

Figure 2.1. Manufacturing strategy assessment

Literature Review

In addition to the availability of biomass

in the U.S. comparing to the fossil fuel, the need for economic, environmental, energy, and national securities is mandatory for any development country (Figure 2.3).

Based on the U.S. and its military concern, different biorenewable pathways focus

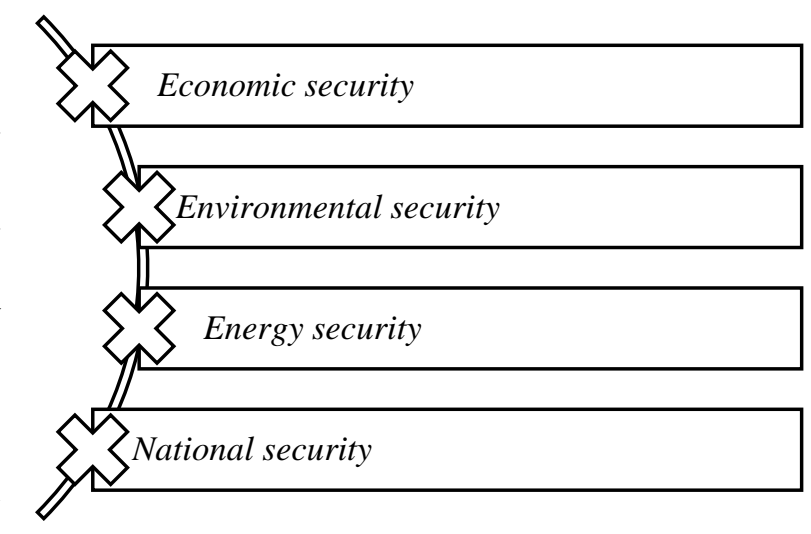

Figure 2.3. Four security types on local, diverse, and sustainable resources of feedstock for biorenewable energy production. In addition, many researchers studied the life cycle cost of different biorenewable pathways (Wright, Daugaard, Satria, \& Brown, 2010). Others looked into the possibility of manufacturing small size, including mobile units with lower costs for renewable energy production (LaClaire, 
Barrett, \& Hall, 2004) which partly serve different security types. All the four security types influence each other where all security types are integrated. All types of security serve the national interest by providing confidence about the energy needed for the life in the states once the nation is free of outside control from other countries.

Economic security focuses on protecting the U.S. jobs and health of the United States economy. This security type forced researchers to work on finding the optimal FPUs' size with the lower cost (Wright \& Brown, 2007; Wright, Daugaard, Satria, \& Brown, 2010).

Environmental security is one of the biggest issues nowadays in the U.S that is focusing on preventing Greenhouse Gas (GHG) emission from fossil fuel combustion. Given that, this emission generates three levels of pollutions, namely: localized non-atmospheric, localized atmospheric and global pollutions (Mateen, \& Brook, 2011). Using biorenewable resources enhance the environmental security due to the low GHG emissions compared to the emissions from producing, transporting, and combustion of fossil fuel (Balat, \& Balat, 2009). There are many arguments in support of biorenewable production. A main argument is that biorenewable is less harmful to the environment compared to fossil fuel (Balat, \& Balat, 2009). According to a study by the National Academy of Sciences in 2009, fossil fuel costs the United States about $\$ 120$ billion a year in health costs, mostly because of thousands of premature deaths from air pollution. This is in addition to what might happen because of the climate change and other natural disasters, where the fuel burning is a key factor in their occurrence. Other types of damages occur due to different types of pollutions. For example, fossil fuel localized nonatmospheric pollutions such as water pollution and deep-water horizon fires is a result of oil spills and leakages cause serious damages to the marine environment and might lead to the death of some marine organisms. Moreover, the polluted water affects humans as well especially if it is 
the drinking source for them. Other examples for localized atmospheric pollutions include the 1995 London Great Smog as well as current Los Angeles smoke problems that directly affect humans' health and cause respiratory diseases. An example of the global pollution is the climate changes and air pollutions course in a city and affecting neighbors' environment (Voegele, 2012).

Another argument in support of this direction states that using biomass to produce bioenergy would motivate farmers to plant more and thus increase their income. Demand for corn for example will mean more corn planting. Even with corn price increases the farmers' profits increases more and more. Thus, this group of people believe biorenewable production enhance environmental security. Democrats represented in Obama administration support this argument and trying to adopt the environmental policy and its application and force the Congress to this direction (Voegele, 2012).

Enhancing environmental security leads to healthy economy for United States. Having more localized fuel resources strengthen the country's economy. Moreover, the internal trade prevents currency form going out to buy the needed energy from other countries. In addition, using biorenewable energy increases the chances of the existence of new sources of livelihood for farmers. Besides, it creates more employment opportunities within the country. Also, environmental non-atmospheric pollutions such as water pollutions could destroy marine life and pollute nearby beaches. Thus, this obscures the tourists from those contaminated places. Consequently, this hurts the country's economic health due to the lack of tourists and low states' income from tourism. Environmental security prevents national harm from global climate change. Finally, by the secured environment, more sustainable and plentiful energy resources will be available from biomass without the risk of climate changes effects, this is known as energy security (Voegele, 2012). 
Energy security focuses on finding more sustainable and plentiful energy resources for the states. In addition, having permanent local sources of energy supports job creation, as well as improves country's economy.

Similar to the economic security and energy security, national security focuses on energy diversification from local sustainable resources, which supports providing job opportunities as well as accelerating the country economy. National security focuses on diversifying local sources of fuel. This perspective is the most important one for the military and thus for the whole state. Therefore, national security is considered as a necessity to maintain the country's survival even with the existence of all other kind of powers. After World War II, the United States recognized the importance of this security type. In the early 1970s, the government of Saudi Arabia stopped its petroleum export to the U.S. due to its support of Israel, which considered as the biggest enemy in the region to Saudi Arabia and other Middle Eastern countries (EIA, 2002). Moreover, another similar problem occurred in the late 1970s during the Iranian revolution. The United States faced this problem again when the Iranian government stopped exporting its petroleum to the United States due to its position opposing of the revolution (EIA, 2002). Parallel in the 1970s, the United States hits the highest petroleum production level and reached the peak oil (Bardi, 2009). After World War II, and later two successive shocks in addition to the substantiation of the peak oil concept, the U.S. government became aware of the urgent need and the danger of its full dependence on foreign oil even with the cheap price comparing to the local produced ones. In addition, it recognized the consequences potential risk to energy, economic, and environmental security of the country.

From the United States perspective, there are three different levels of national security. First, the small-scale that is for military units. Most of modern militaries are entirely mechanized 
and all troop transportation relies on fossil fuels. Moreover, modern militaries rely heavily on electricity. So, the fact that they highly depend on energy and external controls hereby cause grave dangers on army security in addition to the high cost of fuel, as happened during the war of America in Afghanistan where the fuel transporting cost was around $\$ 400$ per gallon. Additionally, one out of twenty-four soldiers was killed during fuel transportation in that war. Furthermore, the high operating cost of the military machinery and equipment affects the environment badly by GHG emissions. Thus, biofuel differs from fossil fuel in the lower cost as well as location diversity, which makes biorenewable energy a major tactical goal for U.S. military. This brings us to the second level of national security, which is the regular military operations that is also concerned with fuel cost, diversity, and sustainability. The third level is the highest one, which is strategic operation that has a global conflict, which in turn supports the previous reasons for renewable energy.

Pyrolysis is a thermo-chemical decomposition of organic material at elevated temperatures. Fast Pyrolysis is defined as a thermo-chemical conversion for producing biofuel from biomass (LaClaire, Barrett, \& Hall, 2004). It is a process for the production of renewable transportation fuels. This process involves the thermal treatment of biomass into liquid bio-oil that is subsequently hydro processed into gasoline and diesel.

The selection of a fast pyrolysis Biomass Processing Modules (BPM) is based on the natural resource base in Iowa, which has made it one of the world's leading producers of food and feed crops. Its resource base also has the potential to grow large quantities of biomass for the production of fuels, energy, and biobased products, as demonstrated by Iowa's leadership in U.S. production of grain-based ethanol. To fully develop this potential, they need to improve (1) utilization of cellulosic feedstock, including wood, corn stover, and switch-grass, and (2) 
distributed processing of these feedstock into biobased products, including drop-in biofuels, commodity chemicals, and bio-materials. Currently, the manufacture of these products depends heavily on crops also used in food production. Although the national resource base of cellulosic biomass is estimated to be in excess of one billion tons annually, it is bulky and highly distributed across the countryside, complicating its collection and delivery to processing facilities (Wright, Brown, \& Boateng, 2008).

The specific BPM, namely, FPU, was suggested to be used as the test-bed for this analysis (Wright, Daugaard, Satria, \& Brown, 2010) (Figure 2.4). Biomass fast pyrolysis is a thermochemical process for the production of renewable transportation fuels, as defined earlier.

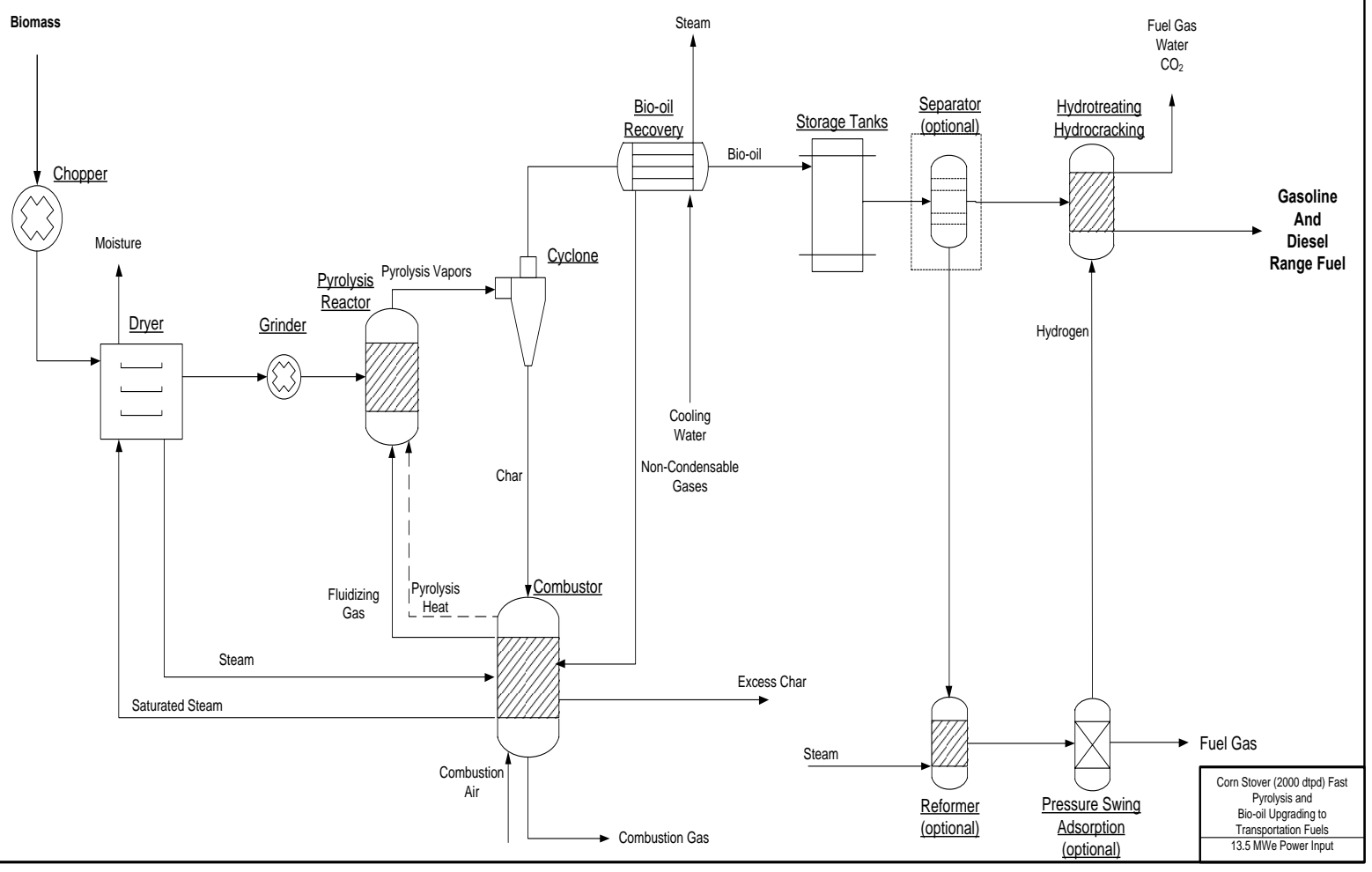

Figure 2.4. Schematic of the biomass fast pyrolysis unit (Wright, Daugaard, Satria, \& Brown, 2010)

It is important to make biofuels a feasible option for renewable energy to increase economic, environmental, energy, and national securities by improved sustainability, as well as 
deliver low cost competitive products to the end-user market (Awudu, \& Zhang, 2012), however, more work is needed to understand the optimal size of FPU (Larasati, Liu, \& Epplin, 2012,).

As described by Wright and Brown (2007), there is an optimal plant size for the lowest unit cost of biobased product. This optimal size is still so large that capital investment for advanced biorefineries are estimated to be as much as a billion dollars. Prior work has also been done to develop frameworks to assess the cost-effectiveness of alternative energy strategies (Qin et al., 2012). The challenge to distributed bioenergy systems is that the unit cost of a production, predicted by conventional cost models, will decrease as the facility size becomes larger (Arrow, 1962; McDonald \& Schrattenholzer, 2001; Tsuchiya \& Kobayashi, 2004). The concept of "economies of scale" states doubling the size of a facility doubles the output, but does not double the construction, operations or maintenance costs. The concept, however does not take into account recent developments in Advance Manufacturing Technology (AMT), such as improved control systems, automation, communication, and data management (Shipp et al., 2012). Some industries, such as aerospace, have begun to explore other design approaches to move away from the "bigger is better" approach (Componation \& Collopy, 2012). In addition, attention is now being paid to non-technical parameters that can drive costs in developing new systems (Hamaker \& Componation, 2010). In the case of processing biomass, the result is complicated because of the transportation costs of highly distributed and low-density materials.

Research has been done about the use of multi-criteria decision analysis approaches to justify FPU's size and AMT implementations. Previous studies in AMT justification approaches have included classic Cost Benefit Analysis (CBA) (Gagnon \& Haldar, 1997), and modified CBA approaches such as the Technology Value Pyramid (TVP) (Tipping \& Zeffren, 1995). Traditional Multi-Criteria Decision-Making (MCDM) (Karsak, \& Ahiska, 2005), Data Envelope 
Analysis (DEA) (Cook, Kress, \& Seiford, 1996), and MAUT (Prasad \& Somasekhara, 1990) approaches have also been explored. Part of this research effort will evaluate alternative justification methods since the selection of a specific decision making approach can influence the results of the analysis (Componation \& Nicholls, 2011). The application of AMT would benefit biofuel production by allowing deployment of relatively small-scale facilities, processing as little as 200 tpd (Wright, Brown, \& Boateng, 2008).

Understanding uncertainties in energy production is useful because it helps build more accurate models. Uncertainties can occur from the energy supply chain itself, but also arise from imperfect knowledge of the critical variables under study, inadequacies of the models from the inherent noise in the data used to populate the model (DOE, 2011). One of the challenges for biofuels is the high level of uncertainty (Table 2.1) in supply, demand, and market price (Kim \& Realff, 2011). Approaches to deal with this uncertainty have included integrating multiple supply chains (Rentizelas, Tolis, \& Tatsiopoulos, 2009), and combined production and logistics strategies (Dunnet, Adjiman, \& Shah, 2008). Mathematical programming models have also been utilized to optimize the biofuel supply chain (Eksioglu, Acharya, Leightley, \& Srora, 2009). Most of previous research of biofuel supply chain are focused on optimization in specific regions using a systems approach rather than looking at the performance of specific supply chain elements (Dal-Mas, Giarola, Zamboni, \& Bezzo, 2011). Prior research has also explored the cost sensitivity of biofuels produced through fast pyrolysis BPM (Wright, Daugaard, Satria, \& Brown, 2010). This research noted that capital costs are particularly important in estimating the cost-effectiveness of the technology. The scope of these research areas is on the biofuels supply chain. 
Table 2.1. Sample Uncertainties Influencing Production of BPM

\begin{tabular}{|c|c|c||}
\hline \hline Uncertainty & Description & Sample Research \\
\hline Demand for food & Uncertainties due to changes in demand for food supplies & (Meyer, 2007) \\
\hline Regulatory environment & Impact of changes in tax incentives on demand for biofuels & (Markanda, \& Pemberton, 2010) \\
\hline Biomass price & Changes in supply yield, location, and price & (Ravindranath et al., 2009) \\
\hline Investment cost & $\begin{array}{c}\text { Capitalization, operations and maintenance cost variability } \\
\text { due to market conditions }\end{array}$ & (Cadre \& Orset, 2010) \\
\hline
\end{tabular}

Each biofuel production stakeholder has different perspective and requirements. These different requirements can make the selection of a biofuel production strategy a challenge. As a result, this research objective, optimizing the production of an FPU, is classified as a complex decision problem that can be solved using an MAUT approach (Min, 1994). The MAUT application follows the same pattern of normal decision-making approaches (Dyer, 2005). Decision makers often have to make decisions with limited information, especially in new areas such as advanced biofuel production. In order to design valid decision-making support systems to assist decision makers in these situations, a detailed analysis on the available information is required. This should also include investigation for factors that influence the decision under different conditions and situations.

It is important to understand stakeholders' perspectives and requirements to make biofuels a feasible alternative for renewable energy; and to increase energy security, improved sustainability, as well as to deliver low cost competitive products to the end-user market (Awudu \& Zhang, 2012); however, little work has been done to optimize the size of biomass processing itself (Larasati, Liu, \& Epplin, 2012).

The Methodology and Proposed Approach

Unfortunately, the persistently high cost of capital and volatility of energy prices conspire to discourage investment in the construction of large-scale bioenergy systems; few investors can 
afford the risk associated with brief downward fluctuations in energy prices and the resulting prospect of being forced to close a billion dollar facility (Figure 2.5). Additional costs can be incurred by selecting an inappropriate supply chain strategy (Harris, Componation, \& Farrington, 2010) to supply raw materials and distribute energy resources. Other difficulties are caused by uncertainties in raw material price, supply, yields, and demand.

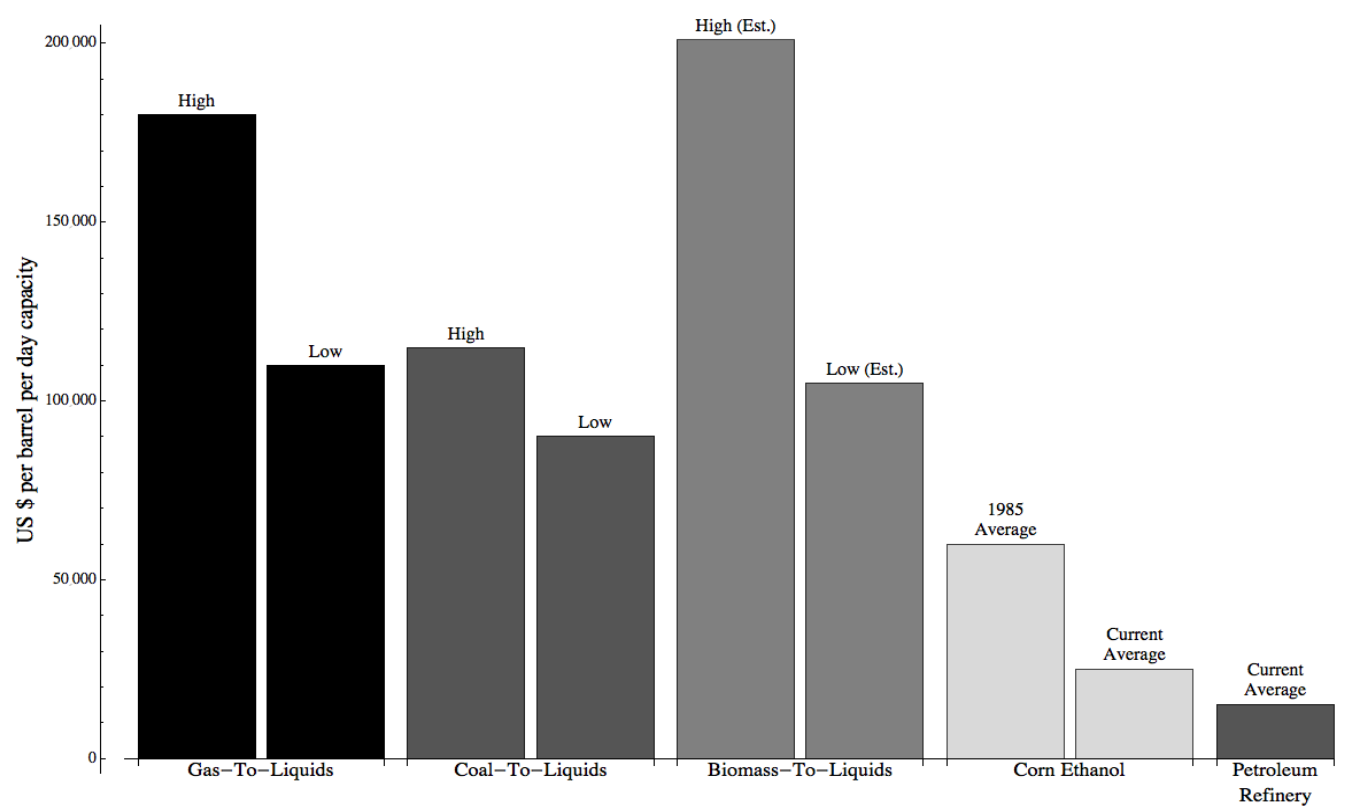

Figure 2.5. Comparison of biofuel and fossil fuel refinery costs on a per energy unit basis

Overcoming the limitations of economies of scale requires a new approach to the production of bioenergy systems. Traditional large production facilities are custom designed and field constructed over many months or even years. Once completed, the number of staff needed to operate them is strongly dependent upon facility size. There is a growing consensus that an alternative approach is needed where the "economy of scale" is replaced by "economy of numbers;" a focus on an energy production strategy that supports a module, distribute approach by using mass-produced, small-unit scale technology (Dahlgren, Lackner, Gocmen, \& van Ryzin, 2012). Replacing field construction with mass production of BPM in highly automated 
Small to Medium sized Enterprises (SME) would promote small-scale distributed biofuel production strategies by using standardized components to reduce cost and improve reliability. BPM would be shipped to a site and become operational in a matter of days or weeks. This would capture the advantages inherent in mass production of other consumer products. Smallscale energy production facilities, once field-assembled, could gain the same economies of scale in staffing as large plants through expanded use of AMT such as automated maintenance and control systems; the energy manufacturing production facility also benefits from AMT through potential reductions in unit production costs. This concept is receiving increasing attention from the biofuels community (Lane, 2012).

Multiple technology pathways can be used to produce biofuels using FPU. The goal of this research is to develop a systems-oriented approach that could be used in later work for the manufacturing, placement, and optimal sizing of an FPUs manufacturing infrastructure that reduces capital costs, investment risk, and is more responsive to changes in energy demands. The selection of specific FPU's size should be accomplished using both quantitative and qualitative factors as typically done in industry (Punniyamoorthy \& Ragavan, 2003) and this will be taken into account in the proposed approach. Quantitative factors revolve around different types of costs, while qualitative factors include flexibility, ease of use, and units' efficiency. Additional factors which be used to select FPU's size will be consistent with prior technology evaluation studies in biofuels, including (1) fast pyrolysis BPM production should match current agricultural output, and (2) fast pyrolysis BPM output should be compatible with present fuel needs (Swanson, Platon, Satrio, \& Brown, 2010). This research planned to be completed through the proposed approach (Figure 2.6): 
1: Identification of BP stakeholders.

2: Identification of BP stakeholders' perspectives \& requirements.

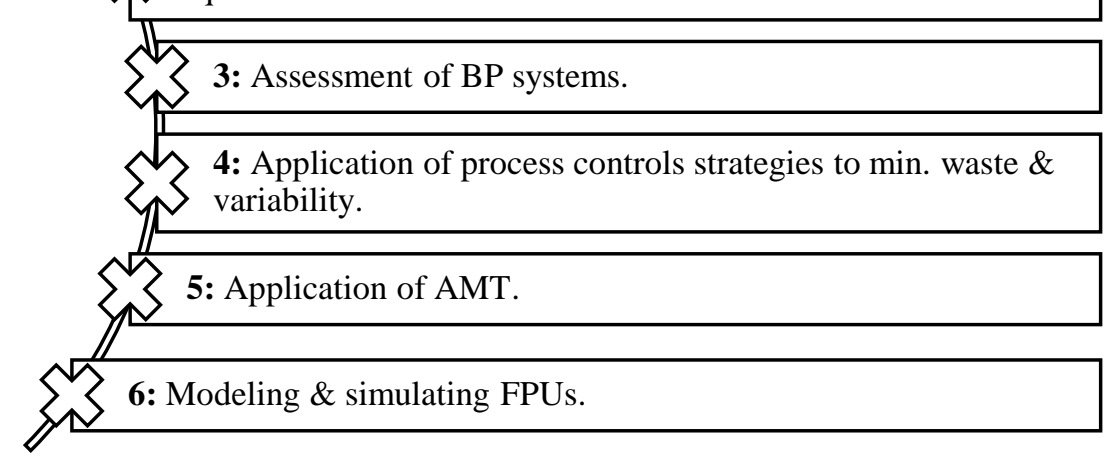

* BP: Biofuel Production

Figure 2.6. Research methodology - Proposed approach

Phase 1: Identification of biofuel production stakeholders: The decision makers identification is the primary step in any decision-making process and it is a critical phase (Bomb, McCormick, Deurwaarder, \& Kåberger, 2007; United States Department of Agriculture, 2012). Based on this stage the different perspectives and requirements for FPUs' size selection will be defined.

Phase 2: Identification of biofuel production stakeholders' perspectives and requirements: The development of a renewable energy system requires identifying and understanding all system stakeholders' perspectives and requirements. This includes energy producers, consumers, government, and society as a whole.

Phase 3: Assessment of biofuel production systems: Optimal sizing renewable biofuel production is needed to minimize capital requirements and reduce risks. This will support investment and development in the industry.

Phase 4: Application of process controls strategies to minimize waste and variability: Production and distribution of affordable energy production systems will require a distributed 
manufacturing system that is based on economics of production rather than economics of scale. Efficient and effective operations are important to reduce costs and improve profitability in the industry. Moreover, new technologies may help reduce the economics of scale so that smaller facilities do not cost more per unit of production than larger facilities (Jack, 2009).

Phase 5: Application of AMT: Reductions in manufacturing costs and improved reliability can be driven by the correct application of technologies to accelerative adoption of renewable biofuel production facilities. This will also reduce the challenges in confronting the economics of scale prevalent in the energy industry.

Phase 6: Modeling and simulation of the biofuel production facilities: Integration of logistics, production, and distribution models will support strategic planning and investment in the energy manufacturing industry.

In addition, market uncertainties should be explored to determine how robust the production standards are to changing market conditions. For phases (2) through (5) in the proposed approach, the sensitivity analysis will play as strong role to examine the decision robustness under different condition changes. For example, market uncertainties could be changes in capital costs, transportation costs, raw material availability and yields, and emergence of complementary and disruptive technologies.

\section{Conclusion}

The implementation of the proposed systems-oriented approach for the manufacturing, placement, and optimal sizing of an FPUs manufacturing infrastructure reduces capital costs, investment risk, and is more responsive to changes in energy demands. This work also assures the achievement of all economic, environmental, energy, and national securities. This system- 
oriented approach is planned to be accomplished through six consecutive phases. These phases are considered as the outcomes for the big-research, which are:

1. Identification of biofuel production's stakeholder.

2. Identification of their perspectives and requirements.

3. Assessment of biofuel production systems.

4. Application of process controls strategies.

5. Application of advanced manufacturing technologies.

6. Modeling and simulation of the biofuel production facilities.

This proposed approach helps to increase the acknowledgment and better understand about the manufacturing of biomass conversion. For engineering managers, this research provides a specific quantitative and qualitative approach or tool to follow as a decision-making process to assess different alternatives for a complex problem in their companies like FBUs' size selection. This approach could be used even with the lack of information needed for the decisionmaking or for novel topics. This approach could be generalized in a future work to be applicable for other complex problems such as other sources for renewable energy production. 


\title{
CHAPTER 3. BIOFUEL PRODUCTION: STAKEHOLDERS' IDENTIFICATION
}

A paper published in Journal of Management and Engineering Integration, Vol. 7, Issue. 1

\author{
Mostafa F. Fawzy and Paul J. Componation
}

\begin{abstract}
This article reports on the identification of Biofuel Production Stakeholders (BPS). Some researchers view BPS as a set of independent biofuel supply chains. Others focused on production without showing the BPS identification. Up to the researchers' knowledge, no research in the literature provide a detailed classification and selection of BPS. Thus, this paper uses a scientific and systematic method to determine the biofuel production stakeholders. Moreover, this methodology could be used as a guideline to build the scientific decision-making team for biofuel production. By application of the modified theory of stakeholder identification and salience, stakeholders are evaluated and classified into eight different groups according to their typologies. Identified stakeholders in this article are often the primary team of BPS, who should be included in any decision-making process related to the biofuel production process.

\section{Introduction}

Biofuel production as an energy resource has become a strategic issue for many counties. In $2011,8 \%$ of the U.S. energy was from renewable resources. The remaining $92 \%$ came from non-renewable resources, including oil, natural gas, nuclear, and coal. Of the $8 \%$ renewable, slightly over half, $51 \%$ was produced from biomass. Within approximately a century, scientists
\end{abstract}


predict that the world would run out of all the non-renewable resources of energy and renewable energy will be required to cover 100\% of the world's energy needs (Wang, 2013).

The U.S. government realized the importance of renewable energy production and has established policies production. In 2005, the government adopted a federal level regulation known as the Renewable Fuel Standard (RFS) calling for the produce of 7.5 billion gallons of renewable fuel by 2012. This policy was revised in 2007 (RFS2) and production of renewable fuel target was increased to 36 billion gallons by 2022 . Additionally, the U.S. government established tax credits for renewable fuel production to motivate investment in this industry (Wang, 2013). To promote further development, all stakeholder perspectives will need to be considered.

Biofuel production and its impact have been studied from many different perspectives. Most of the previous research focused on the economic or the environmental perspectives, or both (An, Wilhelm, \& Searcy 2011; Ayoub et al. 2007; Dwivedi \& Alyavalapati, 2009; EBTP Stakeholders, 2012; Larasati, Liu, \& Epplin, 2012). Other researchers have looked at the legal perspective (Talamini et al., 2012; Youngs 2012), as well as the technical perspective and social impacts of biofuel (Meiera \& Schrödera, 2013; Perimenis et al., 2011).

\section{Study Objective}

The objective of this study is to identify biofuel production stakeholders (BPS) perspectives to provide decision makers with a comprehensive view of all biofuel production requirements and explore how these different perspectives may influence production approaches. The work will also serve as a basis for further exploratory research into renewable energy production strategies. 
This is a methodology to identify, evaluate, and classify different BPS according to five different perspectives of biofuel production. This work is done through a management theory implementation on BPS, whereby different stakeholders' opinions have been explicitly taken into account for the decision-making process for biofuel production. This confirms all decisionmakers contribution in the decision-making, each with the right position.

\section{Literature Review}

Industry, government and researchers have not yet reached a consensus on how to balance the multiple and often conflicting perspectives that influence biofuel production. To move toward this consensus we will need a better understanding of stakeholder groups, what their individual requirements are, and find ways to combine these perspectives so that a alternative strategies for biofuel production can be explored.

Some work has been done to define and identify stakeholders. Turcksin and his colleagues (2010) defined stakeholders in general as "people who have an interest, financial or otherwise, in the consequences of any decision taken." Youngs (2012) pointed out that every person in the world, from their perspective, is considered as a biofuel stakeholder. Some have direct relation or impact on biofuel production and consumption were others have an indirect relation. The European Biofuels Technology Platform (EBTP) defined stakeholders (2012) as "any organization whose commercial or business activities affects, or can be affected directly by the actions taken by the actions or recommendations of the EBTP."

Stakeholders' identification is a very early and important step in any decision-making process (Bomb, McCormick, Deurwaarder, \& Kåberger, 2007; United States Department of Agriculture, 2012). Some studies in this area begin with a listing of stakeholders. Huertas and his colleagues (2010) began the study by mentioned BPS. Youngs (2012) also presented BPS 
and values related to biomass feedstock choices. Another example is the study done by Talamini's and his colleagues (2012) in which they stuied the structure and affect stakeholders' agendas on U.S. ethanol production. Each study focused on understanding BPS rather than how each BPS was identified. For example, Turcksin and his colleagues relied on the stakeholders' groups represented by Turcksin and Macharis stockholders workshop (Turcksin \& Macharis, 2009; Turcksin et al., 2010). This study identified the BPS as seven stakeholders groups for biofuel supply chain, which contained five people in their study.

A review of current and previously completed work shows multiple perspectives used, including financial, businesses (An, Wilhelm, \& Searcy 2011; Ayoub et al. 2007; Dwivedi \& Alyavalapati, 2009; EBTP Stakeholders, 2012; Larasati, Liu, \& Epplin, 2012), research (Perimenis et al., 2011), legal and decision-making, and public and society (Talamini et al., 2012; Mteiera \& Schrödera, 2013; Youngs 2012). The Roundtable on Sustainable Biofuels noted stakeholders include farmers, companies, non-governmental organizations, experts, governments (national \& local), and inter-governmental agencies (Fortin, 2011; Lee, Rist, Obidzinski, Ghazoul, \& Koh, 2011). Scientists, journalists, and policy-makers also are groups of stakeholders identified by Talamini and other (2012). One common theme found in the literature was to identify the BPS based on the needs of the specific study undertaken. This is a logical approach, however it does limit generalization of prior work to address a more system based assessment of biofuel production strategies.

As Dwivedi and Alavalapati stated (2009) in their study, the literature review shows that no study exists that quantifies stakeholders' perceptions regarding biomass-based bioenergy development. 
Theory of stakeholder identification was initiated be Freeman (1984) in this book "Strategic Management: A Stakeholder Approach". At that time, he presented the concept of stakeholders and their importance in the management area (Mitchell, Agle, \& Wood, 1997). Mitchell and his colleagues (1997) modified Freedman's original work by dividing stakeholders' attributes into legitimacy, power, and urgency. Stakeholders with legitimacy are those who influence value identification. This group of people or organizations set the requirements (customers' needs). Stakeholders with power are the group of people or organizations who have influence on value positions, which means the impact on requirements' ranking and priorities. The last attribute is urgency and this group influences value execution. This means this group affects how the solution is done to meet the set requirements. Figure 3.1 shows the three stakeholders' attributes with their overlapping (Mitchell, Agle, \& Wood, 1997).

Mitchell and his colleagues (1997) identified the three classes and overlapping areas. The derivative four classes generated from the intersection areas are also shown in Figure 3.1. Mitchell also identified an eighth class as those who do not have any power, legitimacy, or urgency; this is called Non-stakeholders class. From Youngs (2012) definition of biofuel stakeholders, it seems that this class of stakeholders in not applicable for biofuel stakeholders in general and therefore does part of BPS research. 


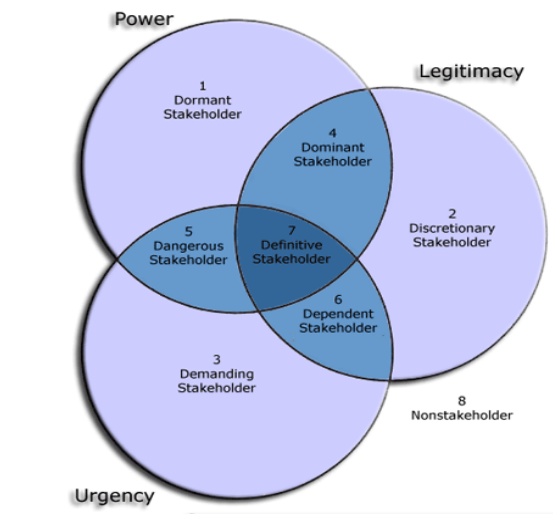

Source (Change Management Toolbook, 2013)

Figure 3.1. Stakeholders' typologies

After that, Mitchell and his colleagues worked on what they call it latent stakeholders. They discussed the eight stakeholders' classes and suggested the stakeholder typology for each class. These typologies are shown in Figure 3.1. Change Management Toolbook represents the eight stakeholders' typologies through the three attributes zones and their intersections as in Figure 3.1 (Change Management Toolbook, 2013). In this figure the eights stakeholders' typology "Non-stakeholder" appears outside all the three attributes.

According to Change Management Toolbook, stakeholders' groups are classified as shown in Table 3.1 (Change Management Toolbook, 2013). This table presents the eight groups and the related attribute(s) for each one of them. Consequently, any stakeholder could be sorted by one of these attributes or by any of the intersected areas between them.

Table 3.1. Stakeholders classes based on attributes

\begin{tabular}{|c|c|c|c|c|}
\hline$\#$ & Stakeholders group & Legitimacy & Power & Urgency \\
\hline 1 & Dormant stakeholders & X & $V$ & X \\
\hline 2 & Discretionary stakeholders & & & $x$ \\
\hline 3 & Demanding stakeholders & $x$ & X & \\
\hline 4 & Dominant stakeholders & $\checkmark$ & 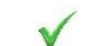 & $X$ \\
\hline 5 & Dangerous stakeholders & X & & $\sqrt{ }$ \\
\hline 6 & Dependent stakeholders & $\checkmark$ & X & $\checkmark$ \\
\hline 7 & Definite stakeholders & $\sqrt{ }$ & $\sqrt{ }$ & $\sqrt{ }$ \\
\hline 8 & Non-stakeholders & X & X & X \\
\hline
\end{tabular}




\section{Methodology}

To reach the research objective, six steps have been followed. First, the literature has been reviewed and the different perspectives for biofuel production have been identified. Then, different BPS teams were identified and linked to their related perspectives. After that, the researchers added some stakeholders' teams with explanations of their importance in such decision-making process of biofuel production that has not been mentioned in the literature. Next, the mind map diagram of BPS with different biofuel production perspectives has been created. Finally, BPS teams have been classified into different classes according to their typologies, which identified by the theory of stakeholder identification.

\section{BPS Identification and Analysis}

In addition to BPS teams identified from the literature, the researchers believe in that contractors, facilities' designer(s), and labors/workers should also be considered as groups of the BPS. Although, Youngs (2012) limited contractors into those who "owns the commodity and pays the farm operator to raise it" while EBTP (2012) limited them into the contracted engineering companies, the researchers suggest that contractors' group should be expanded to include any contractor that involved in the biofuel production process. This definition exceeds Youngs and the EBTP definitions and it is the used one in this article.

In addition, the researchers believe in that facilities' designer(s) should be also a team of the BPS especially at decisions that related to design, layout, and building phase of biofuel production facilities. The facilities' designer(s) participation(s) in decision-making during this phase will be derived from their core work.

Front line employees in biofuel production should contribute in decisions related to their work because they provide important details and ideas related to their daily work that helps the 
decision-making process. Furthermore, first line employees will be affected by these decisions. Their contributions could enhance decisions related to biofuel production and reduce their resistance to implementing the decisions (Al-Amre \& Al-Fowzan, 1998; Jordanian government, Jordanian e-government program, 2007).

The researchers believe that each team has to be classified separately to get its value and attention in the decision-making process for biofuel production. With the researchers' proposed stakeholders' teams plus what have been mentioned previously in the literature as BPS, Figure 3.2 represents the complete BPS diagram. This diagram shows the five perspectives of biofuel production with the related teams of each perspective. As a total, thirty-six BPS teams in this figure are distributed on five perspectives for biofuel production.

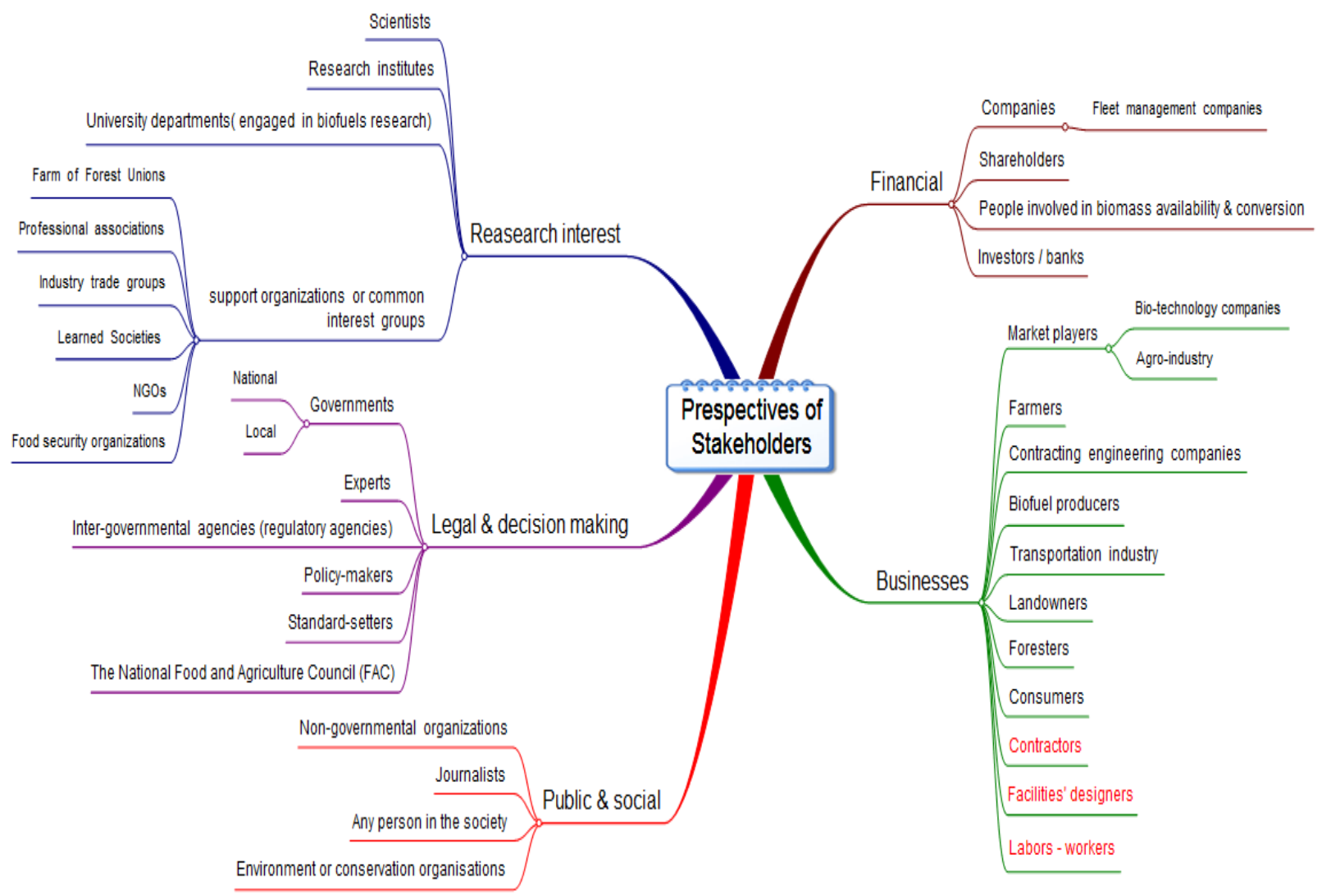

Figure 3.2. Mind map diagram for BPS perspectives and teams 


\section{Result and Discussion}

To recognize BPS teams' priorities, the theory of stakeholder identification has been used with its conducted modification, which is done by Mitchell and his colleagues (1997). To identify BPS teams according to typologies, stakeholders are listed and identified in a matrix as shown in Table 3.2. In this matrix, BPS teams are sorted based on their related perspectives in rows. Then, typologies are listed in the top row. After that, the researchers filled the matrix based on the attributes definitions and their knowledge about each team nature of the work in biofuel production.

In this matrix, thirty-six BPS teams are classified into seven different typologies and from five different perspectives for biofuel production process.

Table 3.2. Stakeholders Typologies' Matrix

\begin{tabular}{|c|c|c|c|c|c|c|c|c|c|}
\hline \multirow[b]{2}{*}{ 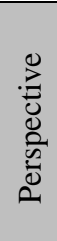 } & \multirow[b]{2}{*}{ BPS Teams } & \multicolumn{8}{|c|}{ Stakeholders' Typologies } \\
\hline & & 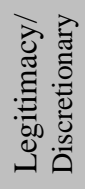 & 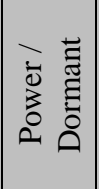 & 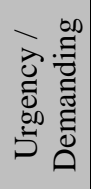 & 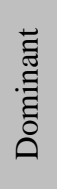 & 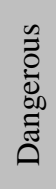 & 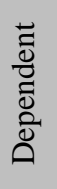 & $\begin{array}{l}\stackrel{\mathscr{\Xi}}{\Xi} \\
\text { صू }\end{array}$ & 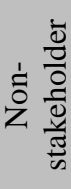 \\
\hline \multirow{4}{*}{ 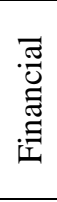 } & Companies (Fleet management companies) & & & & & & & $\sqrt{ }$ & \\
\hline & Shareholders & & & & & & & $\sqrt{ }$ & \\
\hline & People involved in biomass availability $\&$ conversion & & & & $\sqrt{ }$ & & & & \\
\hline & Investors / banks & & $\sqrt{ }$ & & & & & & \\
\hline \multirow{12}{*}{ 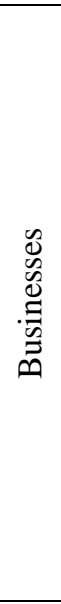 } & Bio-technology companies & & & $\sqrt{ }$ & & & & & \\
\hline & Agro-industry & & & $\sqrt{ }$ & & & & & \\
\hline & Farmers & & & $\sqrt{ }$ & & & & & \\
\hline & Contracting engineering companies & & & $\sqrt{ }$ & & & & & \\
\hline & Biofuel producers & & & $\checkmark$ & & & & & \\
\hline & Transportation industry & & & $\checkmark$ & & & & & \\
\hline & Landowners & $\checkmark$ & & & & & & & \\
\hline & Foresters & $\sqrt{ }$ & & & & & & & \\
\hline & Consumers & $\sqrt{ }$ & & & & & & & \\
\hline & Contractors & & & & $\checkmark$ & & & & \\
\hline & Facilities' designers & & & $\checkmark$ & & & & & \\
\hline & Labors - workers & & & $\sqrt{ }$ & & & & & \\
\hline \multirow{2}{*}{$=$} & Scientists & & & $\sqrt{ }$ & & & & & \\
\hline & Research institutes & & & $\sqrt{ }$ & & & & & \\
\hline
\end{tabular}




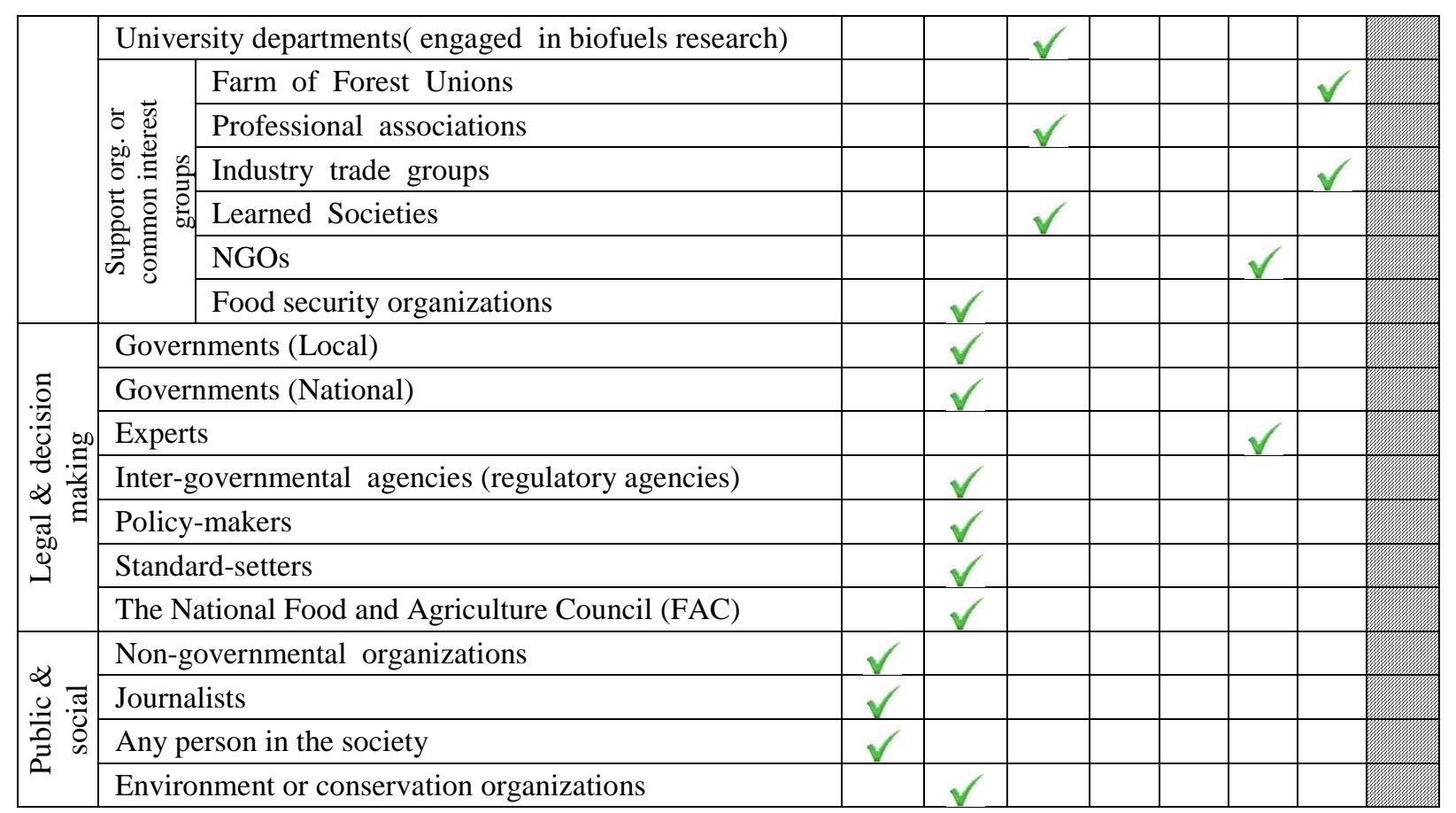

As a result, out of these thirty-six teams, six are classified as the ones who have the legitimacy, nine as having the power, and thirteen as having the urgency. Moreover, by looking to the teams who combine two attributes together it was found that two groups are classified as having both legitimacy and power. These groups called Dominant groups. Likewise, two groups are classified as having both legitimacy and urgency, which are called Dependent groups. On the other hand, none of the groups is classified as having both power and urgency, which is called Dangerous group. Similarly, none of the groups is classified as a non-stakeholder group. For this group, the result is expected due to the Youngs (2012) standpoint when he indicated that every person in the world is considered as a biofuel stakeholder. As he mentioned, some of them has a direct relation or impact on biofuel production and consumption were others have an indirect relationship. Thus, the researchers blocked out this column of stakeholders' typology in the analysis matrix. The result of BPS teams' distribution among the eight typologies is summarized in Table 3.3. 
Table 3.3. Summarized BPS teams' typologies

\begin{tabular}{|c|c|c|c|c|c|c|c|}
\hline $\begin{array}{c}\text { Legitimacy/ } \\
\text { Discretionary }\end{array}$ & $\begin{array}{c}\text { Power / } \\
\text { Dormant }\end{array}$ & $\begin{array}{c}\text { Urgency / } \\
\text { Demanding }\end{array}$ & Dominant & Dangerous & Dependent & Definite & $\begin{array}{c}\text { Non- } \\
\text { stakeholder }\end{array}$ \\
\hline 6 & 9 & 13 & 2 & 0 & 2 & 4 & 0 \\
\hline
\end{tabular}

The pie chart in Figure 3.3 represents the percentage of BPS distribution among the eight typologies. From this pie chart as well as from the previous results table, it is noticed that the biggest group that will be affected by biofuel production is those teams who have the urgency (36.11\%). After that, comes the group of people and / or organizations who have the power. This class represents exactly $25 \%$ of all BPS. The percentages of each stakeholders' typology is illustrated in Figure 3.3.

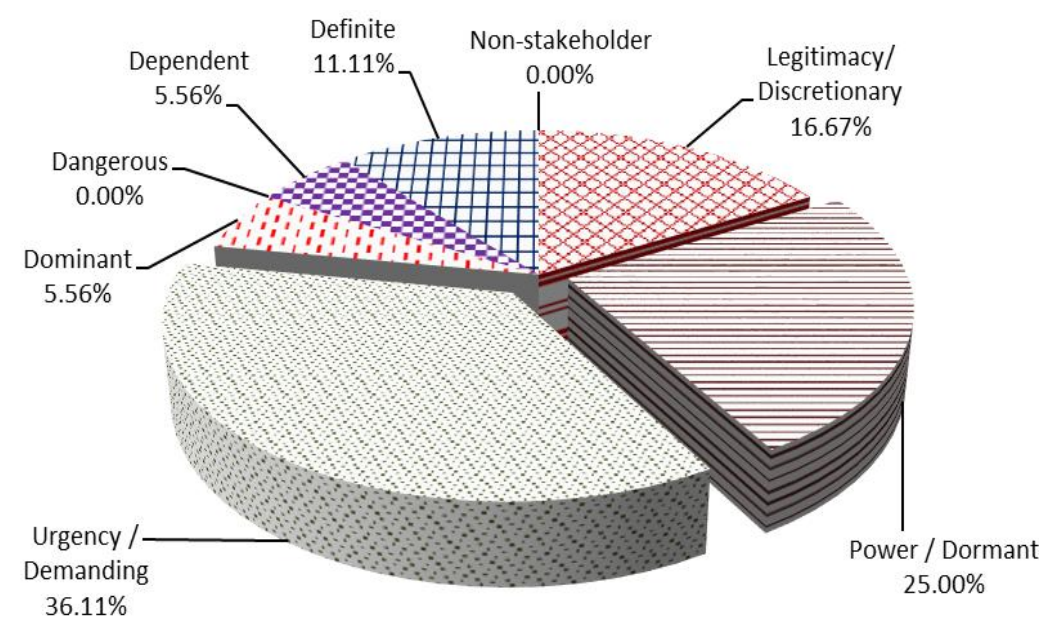

Figure 3.3. BPS distribution among typologies

Although, BPS identification is just an early step in the decision-making, however, upon the serious consideration of this step affects the validity of the decision taken. The amount of work in this step should rely on the size and importance of the decision. BPS could assess the decision according to their typologies. Their participation could be done through a questionnaire, workshop, meeting, or any other method, each according to the role and effectiveness in decision-making. 


\section{Assumptions and Limitations}

This study was conducted under three assumptions. First, the five perspectives educed from the literature are the only ones for the biofuel production. Second, the thirty-three BPS teams from the literature plus the three suggested teams by the researchers are considered the BPS teams. Last, the assessment and classification of BPS teams was done based on the researchers' understanding and belief of the nature and the role of BPS in biofuel production process. Thus, this is the appropriate distribution for them in to the typologies' matrix.

\section{Conclusion}

In conclusion, this work proposed a supposed BPS teams' evaluation and classification in the eight typologies teams. In addition, these teams have been classified according to their perspectives of biofuel production. This was done through the application of theory of stakeholder identification and its modification that done by Mitchell and his colleagues (1997). This work highlights the importance of stakeholders' identification, classifications, and the impact of this on the decision-making in general and especially for biofuel production process. Instead of relying on any researchers' point of view or making workshops for a group of the BPS teams as what has been previously done, this paper used a scientific theory in management science as a method to present and classify BPS. This article provides a scientific method to assess BPS and classify them into several categories according to the extent of their influence and their perspectives about the subject. The results of this study could be used to get biofuel production decisions. Finally, this study might help to identify those who are responsible for decision-making and their influence into the decision. 


\section{Recommendations and Future Work}

For future work, this analysis could be re-conducted with a group of Subject Matter Experts to study the result's robustness or to have more validated analysis for the BPS classifications and typologies. Moreover, some other BPS teams if found could be added to the mind-map chart as well as to the analysis matrix. The additional teams could be evaluated and analyzed by using the same methodology applied in this study. Future work should be conducted to classify efficient methods of all BPS(s) involvement in decision-making process, where different tools and techniques could be used to get each BPS voice. Finally, the same study could be applied to identify other biomass products stakeholders. 


\title{
CHAPTER 4. BIOFUEL PRODUCTION: UTILIZING STAKEHOLDERS' PERSPECTIVES
}

\author{
A paper published in Engineering Management Journal, Vol. 27, Issue. 2
}

\author{
Mostafa F. Fawzy and Paul J. Componation
}

\begin{abstract}
The use of biofuels as a replacement for fossil fuels is growing in the United States and other countries in part because of economic and environmental concerns. One of the technologies for biofuels production is fast pyrolysis; however, to increase manufacturing of fast pyrolysis units, a better understanding of stakeholders' requirements and perspectives is needed. This is a complex decision problem. Due to the diversity of perspectives, each group of stakeholders has their own unique requirements, which in total will determine the right manufacturing approach. Previous studies either investigated optimal sizing from a single viewpoint or have combined a subset of perspectives. This study applies multiple tools to develop a more comprehensive view of stakeholders' perspectives. Individual subject matter experts were asked to review and prioritize a set of requirements that reflected different stakeholders' perspectives, including economic, environmental, technical, social, and legal. The perspectives were then used to analyze multiple fast pyrolysis units to determine which size was the most effective in meeting the perspectives in total. The analysis indicated that the smallest unit, able to process an average of 50 tons per day, is the best alternative when viewed from the economic, technical, social, and
\end{abstract}


legal perspectives. However, when viewed from the environmental perspective, a medium-sized unit, able to process in the range of 200-500 tons per day, is the best alternative. This work provides the basis for further discussions about the individual perspectives, including the economic and environmental perspectives of biofuel production. Potential avenues for further work in assessment of stakeholders' requirements are also noted.

Keywords: Decision-making, biofuel, pyrolysis, stakeholder requirements

EMJ Focus Areas: Program \& Project Management, Quantitative Methods \& Models, Strategic Management

\section{Introduction}

In 2012, approximately $91 \%$ of U.S. energy production came from non-renewable sources, the majority of which was fossil fuels including oil, natural gas, and coal. The remaining $9 \%$ came from renewable sources, $49 \%$ of which was from biomass. A large portion of this biomass is converted to biofuels (EIA, 2013). Increasing the use of biofuels is important because scientists estimate that the world will run out of non-renewable fossil fuels within the next century (Shafiee \& Topal, 2009).

President Obama's 2013 State of the Union address identified clean energy technologies as an important pillar for future economic development in the U.S. A new field of research is the execution of this national priority - the manufacture of devices that produce clean energy. The Department of Energy refers to this as "energy manufacturing" (U.S. Department of Energy, Office of Energy Efficiency \& Renewable Energy, 2014), and evidence of the growth of this area can be seen by the increased number of NSF research initiatives and workshops supporting this field (Georgia Tech Manufacturing Institution, 2009; Georgia Tech Manufacturing Institute, 
2011). Identifying and understanding different stakeholders' perspectives is an important first step to develop a sustainable research agenda in energy manufacturing.

The wide range of stakeholders in the public and private sectors has a significant effect on which renewable energy sources are developed, where they will be developed, and the design of the energy production system to fit energy demands. These questions are of particular significance in biofuel production. Conventional cost models predict that the unit cost of energy production will decrease as facility size becomes larger (Arrow, 1962; McDonald \& Schrattenholzer, 2001; Tsuchiya \& Kobayashi, 2004). In the case of biofuel production, facility sizing becomes more complicated because of the transportation costs of highly distributed and low energy density materials. Crude oil refineries in the U.S. average over 126,000 Barrels Per Day (bpd), and the largest U.S. refinery can process over 560,000 bpd (3\% of the total U.S. refining capacity). By comparison, the capacity of bio-refineries is approximately 10,000 bpd. This large difference is due in part to significant logistical challenges faced by biomass supply chains that negate capital savings from economies of scale (Richard, 2010). There is an optimal plant size for the lowest unit cost of biofuel production (Wright \& Brown, 2007). However, this optimal size is still so large that capital investments for advanced bio-refineries are estimated to be as much as a billion dollars, increasing the difficulty in raising capital and increasing investment risk.

There have been advances that may help address the challenge of optimal sizing of biofuel production. New approaches may help reduce the economies of scale so that smaller facilities do not cost more per unit of energy produced than larger facilities do. Some industries, such as aerospace, have begun to explore other design approaches to move away from the "bigger is better" approach (Componation \& Collopy, 2012). 
Advanced energy manufacturing technologies can be used to improve the biofuel industry, specifically, production of an optimal-sized Fast Pyrolysis Unit (FPU). This article reports on progress in the initial phases of a larger research effort on how engineering managers facing this class of problem can use decision-making approaches to deal with multiple, oftenconflicting, stakeholder requirements. In this work, stakeholders' requirements for the biofuel production industry were first identified through a current review of research publications. Second, requirements were clustered to define stakeholders' perspectives and then verified by Subject Matter Experts (SME). Third, the SMEs prioritized the requirements and used the prioritized list to assess three different FPUs to determine how different sizes of FPUs could meet these perspectives. A sensitivity analysis was then conducted to determine how robust the final recommendation is to changes in stakeholders' priorities. This process, applied to biofuel production, is also applicable in a range of similar open-ended problems that are common in engineering design. Problems characterized by limited information, multiple stakeholders, and conflicting requirements are often solved by decision-making processes such as Multi-Attribute Utility Theory (MAUT).

\section{Literature Review}

There are multiple stakeholders in renewable energy. Many advocate increasing support for renewable energy to improve energy independence and minimize the impact of energy production on the environment. For example, the U.S. government recognized the importance of renewable energy and adopted policies to increase its production. In 2005, the Renewable Fuel Standard (RFS) was passed to set a goal of 7.5 billion gallons of renewable fuel by 2012. In 2007 the policy was revised (RFS2), and the U.S. government increased the goal to 36 billion gallons 
by 2022. Additional tax credits have also been allowed for renewable energy to motivate investors (Schnepf \& Yacobucci, 2010).

In general, stakeholders looking at renewable energy make their evaluations based on efficiency, availability, costs, emissions, and other performance requirements (Karvetski, Lambert, \& Linkov, 2010). Researchers in biofuels also note requirements from contracts and regulations, raw material sources, as well as social, cultural, and political sources (Youngs, 2012). Stakeholders' priorities vary but it may be possible to model their requirements to develop a renewable energy manufacturing strategy. Youngs (2012) suggested a map for stakeholders' perspectives and their influence on biomass source selection, which may be used as an example of this modeling approach (Figure 4.1).

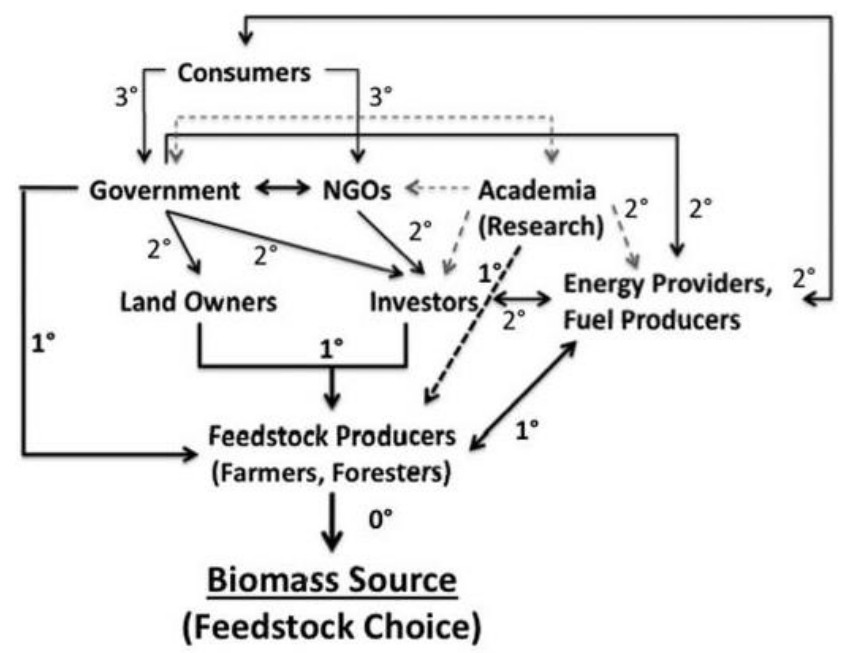

* The Arrows indicate Direction of Stakeholder Influence and the Number on Each Link Indicates the Degrees of Separation between the Stakeholder and the Feedstock Source. Dashed Lines note the Role of Academia in Biofuel Research. Source: (Youngs, 2012)

Figure 4.1. Map of stakeholders' interactions that influence biomass feedstock choices

There have been numerous publications in the past two decades that have identified a range of stakeholders' requirements for the production and use of biofuels. Economic 
requirements are commonly mentioned. These include costs related to production and operations, materials, transportation, and capital costs. Stakeholders' requirements include notes on the capital risks of investing in biofuels (Wright, Brown, \& Boateng, 2008) and facility size requirements (Jack, 2009). Stakeholders generally support biofuel development when there is a clear opportunity for profit (Michalopoulos, Landeweerd, Werf-Kulichova, Puylaert, \& Osseweijer, 2011). The work that addresses environmental requirements focuses primarily on $\mathrm{CO}_{2}$ and GHG emissions. The opinions on biofuel, both positive and negative, are influenced by differences in stakeholders' perspectives (Michalopoulos, Landeweerd, Werf-Kulichova, Puylaert, \& Osseweijer, 2011). Understanding these requirements is also important to make biofuels a feasible alternative to increase energy, national, and environmental security, as well as to deliver low-cost competitive products to market (Awudu \& Zhang, 2012).

There are multiple technologies that can be used for the production of biofuels: One is pyrolysis, which refers to the thermochemical decomposition of organic material at elevated temperatures. Ancient Egyptians used pyrolysis to produce tar for caulking boats. More recent uses of this technology include charcoal and coke production. In the 1980s, researchers discovered a method to improve the yield of the process by indirectly heating and rapidly condensing the biomass (LaClaire, Barrett, \& Hall, 2004). An FPU is used for the thermochemical conversion of biomass to biofuel.

An FPU was selected as a research artifact in this study to encourage development of the natural resource base in Iowa (see Figure 4.2) (Wright, Daugaard, Satria, \& Brown, 2010), which has made it one of the world's leading producers of food and feed crops. The state produces large quantities of cellulosic biomass for the production of fuels and bio-based products, as demonstrated by Iowa's leadership in U.S. production of grain-based ethanol. Although Iowa can 
support significant production of biomass, the material is bulky and highly distributed across the countryside, complicating its collection and delivery to processing facilities (Wright, Brown, \& Boateng, 2008). The production and use of FPUs should match current agricultural output and present fuel needs (Swanson, Platon, Satrio, \& Brown, 2010).

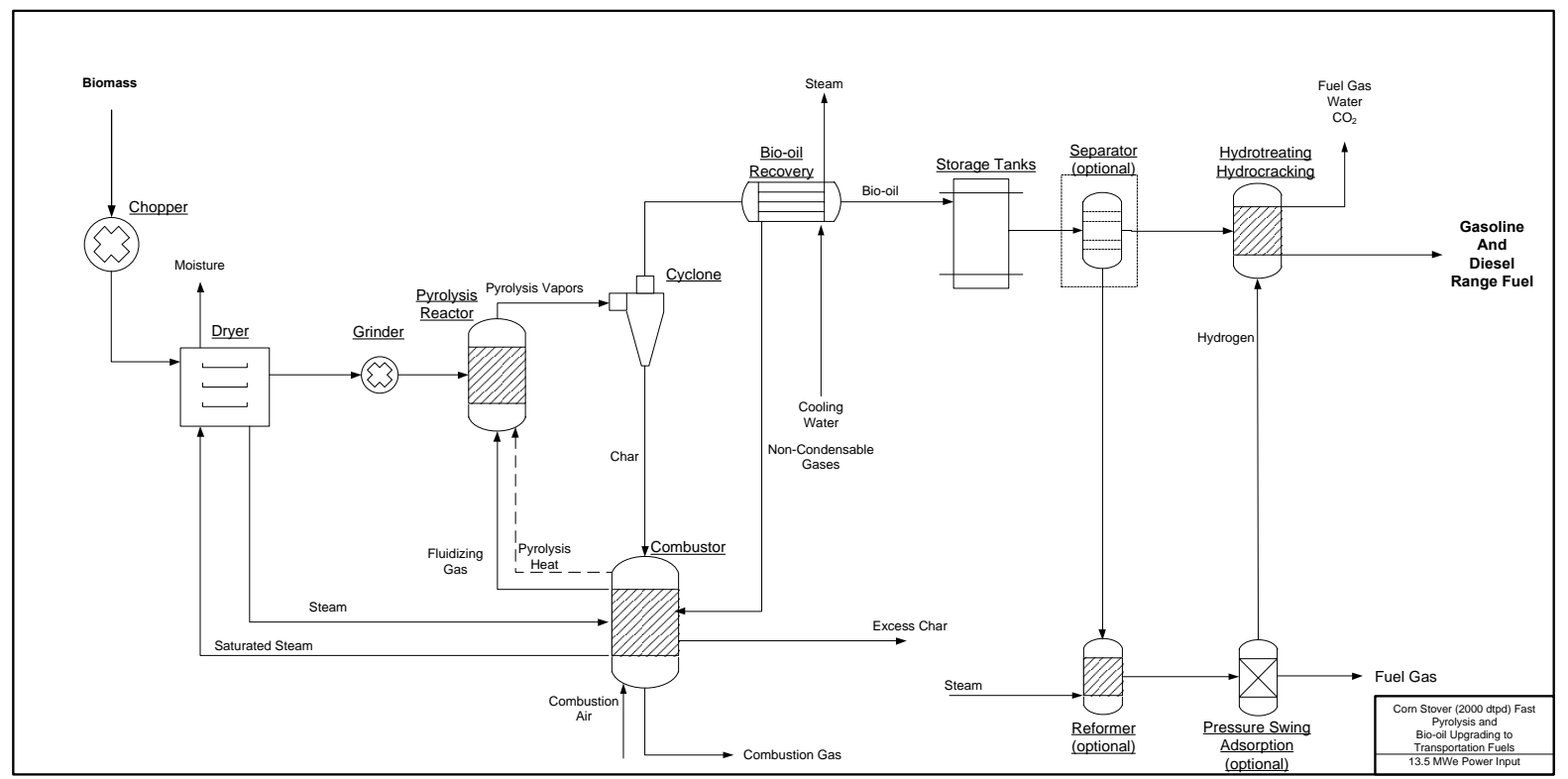

Source: (Wright, Daugaard, Satria, \& Brown, 2010)

Figure 4.2. Schematic of the biomass fast pyrolysis unit

Limited work has been done to optimize the size of biomass processing itself (Larasati, Liu, \& Epplin, 2012) and few studies have analyzed bioenergy production in depth (Dwivedi \& Alavalapati, 2009). Additional effort is needed to understand what the optimal size of a biomass processing facility should be (Larasati, Liu, \& Epplin, 2012). Part of the challenge is to understand the multiple and often conflicting stakeholders' perspectives and requirements. Researchers have noted that the optimal size of a biofuel unit depends on many variables, not least of which is the issue of transportation costs (Wright \& Brown, 2007; Larasati, Liu, \& Epplin, 2012). 
Previous research has used different size biofuel facilities. A typical corn ethanol plant consumes 2,000 tons per day (tpd) of biomass (Wright, Brown, \& Boateng, 2008). Many current research studies use 2000 tpd as their base case to allow for easy comparison with other work even though this may not be the optimal size for all technologies (Wright, Brown, \& Boateng, 2008; Anex, et al., 2010; Swanson, Platon, Satrio, \& Brown, 2010; Wright, Daugaard, Satria, \& Brown, 2010). A medium-sized unit that consumes 200 to 500 tpd of feedstock has been proposed as an alternative that could be supported by a small group or a cooperative of farmers (Ringer, Putsche, \& Scahill, 2006; Wright, Brown, \& Boateng, 2008; Wright, Daugaard, Satria, \& Brown, 2010). The capacity and costs for this size is comparable to the early ethanol and biodiesel plants. The smallest size unit proposed consumes $50 \mathrm{tpd}$ and researchers advocate this as a viable alternative because it can be mobile rather than built in a fixed location. FPUs of this size have been shown to be technologically feasible (LaClaire, Barrett, \& Hall, 2004).

Due to different stakeholders' perspectives, the optimal sizing of FPUs can be classified as a complex decision problem that should be solved using a MAUT approach (Min, 1994). All decision-making approaches should have the following steps: objective identification, identification of two or more alternatives, modeling, result analysis, and sensitivity analysis. The MAUT application follows the same pattern followed by other decision-making approaches (Dyer, 2005). Although each decision-making approach uses different terminologies to name the process stages, the essence of these stages is the same (DCLG, 2009; Chelst \& Canbolat, 2011).

MAUT is more capable of handling practical-sized problems than other methods, such as AHP, because MAUT can handle more alternatives than the latter (Smith \& Speiser, 1991). Due to the lack of accurate data availability for FPUs and the novelty of this research area, advanced mathematical decision-making tools cannot be used in this research. The Pugh concept matrix is 
an alternate tool that could be used to determine the right direction of the facility size for biofuel production without complex mathematical formulas (Cervone, 2009a). The Pugh concept is a type of a paired comparison analysis (Cervone, 2009b) that can be used as a decision tool when there is little or no data for making a decision (Cervone, 2009a). It is a useful decision-making technique for biofuel production stakeholders who are challenged with a problem that has multiple perspectives and requirements (Pugh, 1991). Furthermore, the Pugh concept is a technique of multiple criteria decision analysis inherent in MAUT (Dyer, 2005). This technique is useful for decisions that deal with quantitative and qualitative factors, in addition to uncertain environments and risky situations (Min, 1994; Cervone, 2009a). It compares different alternatives based on known criteria. Moreover, it can be used in situations where there are multiple factors that may significantly affect the decision (Cervone, 2009a). This technique can be used for unlimited evaluation measures.

Smaller-scale technology has not been proven to be the right solution in all situations, but engineers are encouraged to think small in design and manufacturing perspectives (Dahlgren, Lackner, Gocmen, \& van Ryzin, 2012). There has been some research looking at alternative sized FPUs, although most of the work has been in Europe (Dwivedi \& Alavalapati, 2009). If FPUs are to be part of the larger energy infrastructure, then more work is needed. Specifically, this research helps identify and prioritize stakeholders' perspectives. It also presents a method to analyze these perspectives in a rigorous manner. This method could also have a role in the analysis of stakeholders' requirements for FPUs' manufacturing.

\section{Methodology}

The research method was designed to support the goal of enabling further development of an approach for the manufacturing, placement, and optimal sizing of FPUs for infrastructure of 
biofuel energy production. In prior work, stakeholders in the biofuel industry were identified (Fawzy \& Componation, 2014). The aim of this paper is to identify stakeholders' perspectives based on reported requirements, analyzing FPUs to determine how they meet those perspectives, and determining how sensitive the analysis is to changes in stakeholders' priorities (Figure 4.3).

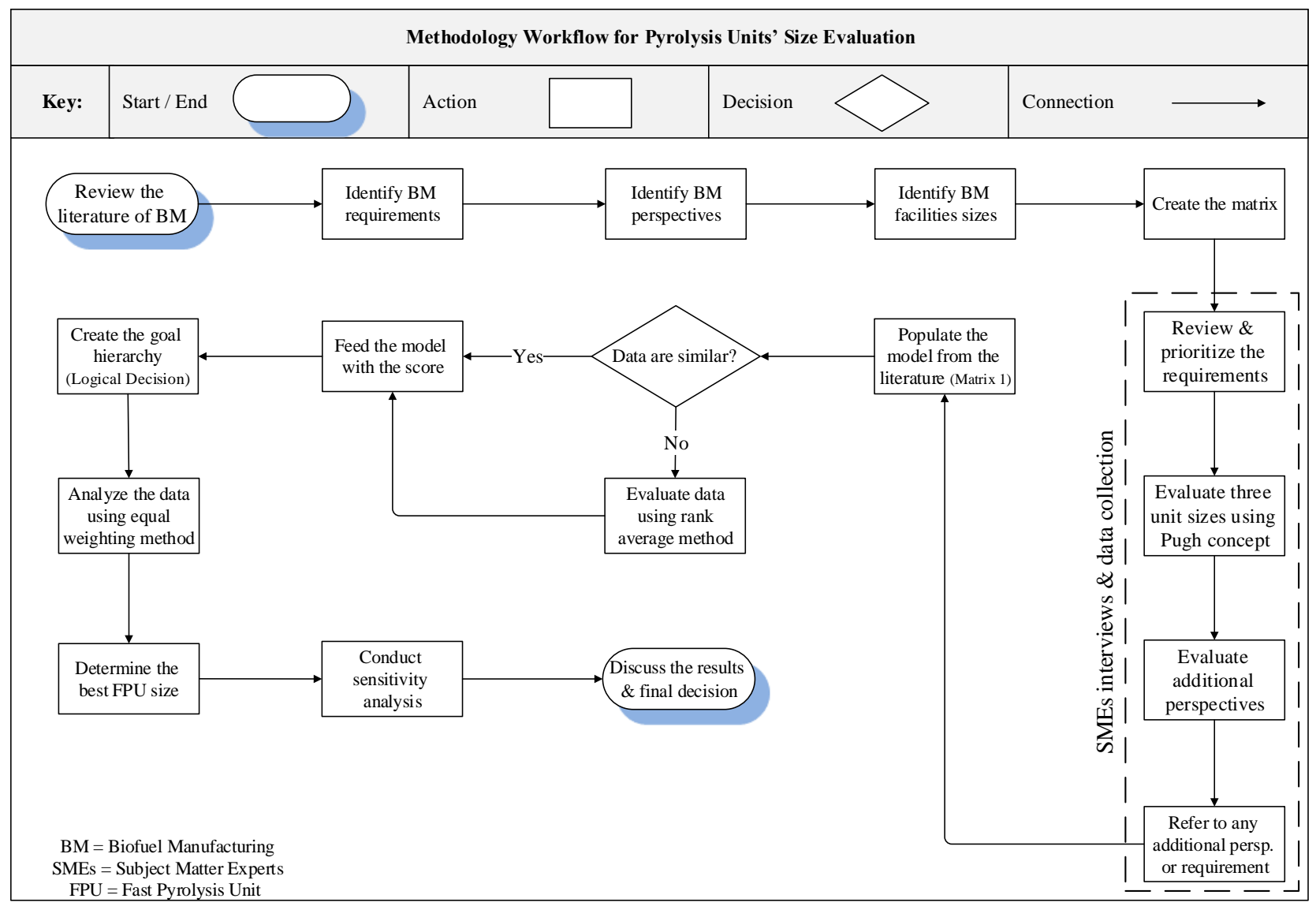

Figure 4.3. Study methodology

The research begins with a review of the last twenty years of peer-reviewed publications on the biofuel manufacturing industry to identify stakeholders' requirements based on different interests or perspectives. From the published literature, a draft list of stakeholders' requirements was developed with individual documents often providing multiple references. Requirements were then ranked based on the number of times each was referenced in the literature. To control 
the size of the requirements list, individual requirements referenced five or fewer times since 1996 were excluded.

The final list of stakeholders' requirements was then reviewed by a team of ten SMEs for completeness. The SMEs were recommended based on consultation with the management team from the Iowa State University Bioeconomy Institute, and included academic researchers and industry representatives. The SMEs' experience ranged from 3 to 38 years, with an average of 13 years. SMEs also had advanced degrees in Food, Agricultural, Biorenewable Resources and Technology, Biological Engineering, Mechanical Engineering, Industrial Engineering, and Organic Chemistry. All SMEs had prior experience in, or were currently involved in, biofuels research. SMEs were interviewed individually and their responses were recorded by the interviewer. The SMEs were asked to place each requirement in one of three groups: High importance, medium importance, and low importance. The interviewer explained to the SMEs the three levels of evaluation as follows:

- High importance: A small change in this requirement would have a significant measurable impact on the recommended biofuel production strategy.

- Medium importance: A change in this requirement would have a measurable impact on the recommended biofuel production strategy.

- Low importance: A change in this requirement may influence the recommended biofuel production strategy.

Each SME reviewed and prioritized each item (high, medium, low) on the stakeholders' requirements list. Then, each SME evaluated the FPUs against each individual requirement using the 2,000 tpd FPU as the base case since this is the most common size unit researched. The SMEs looked at the 200-500 tpd FPU and were asked if it would have an advantage, be the same, 
or be at a disadvantage in meeting this requirement when compared to the 2,000 tpd FPU base case. A five-point scale was used: big advantage, advantage, same, disadvantage, and big disadvantage. During this data collection process, SMEs were also able to provide comments to the researchers. This process was then completed with the 50 tpd FPU.

To determine which FPU provided the best fit for stakeholders' requirements and perspectives, the raw data collected from SMEs on prioritization of the requirements were converted to numerical scales. The prioritization or weight of the requirements was performed using Equation 4.1.

$$
\text { Requirement's weight }=\frac{3 *(\# \text { of SMEs } 1)+2 *(\# \text { of SMEs } 2)+1 *(\# \text { of SMEs } 3)}{\text { Total number of } S M E S}
$$

Where:

SMEs 1: is the group of those who prioritized the requirement's importance as high.

SMEs 2: is the group of those who prioritized the requirement's importance as medium.

SMEs 3: is the group of those who prioritized the requirement's importance as low.

The raw data on the performance of each FPU against the base case was then converted to a numerical score.

Scores for the 200-500 tpd and 50 tpd FPUs were calculated by summing the products of the requirements' weights and scores. The 2,000 tpd FPU score is " 0 " because it is the base case. Higher scores for the other FPUs indicate a better fit to stakeholders' requirements and lower scores indicate a poorer fit.

Concerns were raised that conducting a sensitivity analysis of the three different FPUs using the prioritized stakeholders' requirements list could be perceived as biased. The majority of requirements were seen as economic, thereby making a balanced analysis more challenging. 
To address these perspectives, the requirements were grouped into the five perspectives mentioned in the literature review: economic, environmental, technical, social, and legal. Weighting of the perspectives was performed by normalizing the weights of the individual requirements and summing the weights for the requirements in each perspective. The initial analysis was re-run using normalized weights to confirm that the same FPU was recommended as the original analysis. The sensitivity analysis was conducted on the model using the Logical Decisions software (Biggam, 2011). This type of analysis explores the response of the overall utility of alternatives to changes in the relative importance (weight) of each requirement (Biggam, 2011).

\section{Results}

This study started by identifying stakeholders' requirements from the literature reviewed for this work. Individual publications that included one or more observations relevant to this research were reviewed. Of the 353 observations noted across papers, a total of 202 (57\%) were related to the economics of biofuels. References that included environmental requirements, a commonly-mentioned perspective when discussing biofuels, totaled 52 (15\%). A total of $33(9 \%)$ observations were also found that noted legal requirements, including government policies, taxes and incentives. More detailed information about all papers reviewed is shown in Table 4.1. 
Table 4.1. References for Stakeholders' Requirements for Biofuel Production

\begin{tabular}{|c|c|c|c|c|c|c|c|c|c|c|c|c|c|c|c|c|c|}
\hline \# & Requirement $\quad$ Year & ஃั & ฮ్ & ชి & ஜి & ఫ্ণ & ڤి & ஜ̊ & હ) & 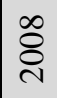 & oे & 일 & $\overline{\mathrm{i}}$ & 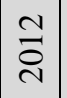 & $\stackrel{m}{\stackrel{n}{2}}$ & 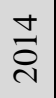 & $\stackrel{5}{\stackrel{\pi}{0}}$ \\
\hline 1 & $\mathrm{CO}_{2} \& \mathrm{GHG}$ emissions & 1 & 1 & 1 & & & 1 & 2 & 1 & 1 & 3 & 4 & 7 & 4 & 2 & & 28 \\
\hline 2 & Land use exchange & 1 & & & & & 1 & & & & & 1 & 2 & 1 & 1 & & 7 \\
\hline 3 & Resources saved & 1 & & & & & 1 & 1 & 1 & & & 4 & 5 & 3 & 1 & & 17 \\
\hline 4 & Design cost & & & & & & & & 1 & & & 1 & 2 & 3 & & & 7 \\
\hline 5 & Capital cost & 1 & 1 & 1 & & 1 & & & 2 & & 1 & 2 & 6 & 4 & 1 & & 20 \\
\hline 6 & Number of pieces of equipment & & & & & & & & & & & & 1 & 2 & 1 & & 4 \\
\hline 7 & Equipment cost & & & 1 & & & & & & & 2 & 2 & 3 & 3 & 2 & & 13 \\
\hline 8 & Labor cost & & 1 & 1 & & 1 & & & 1 & & & 1 & 6 & 5 & 2 & & 18 \\
\hline 9 & Production / Operation cost & 1 & 1 & 1 & & 1 & 1 & 1 & 3 & & 4 & 1 & 9 & 4 & 2 & 1 & 30 \\
\hline 10 & Materials cost (feedstock) & & & 1 & & & 1 & & 2 & & 2 & 3 & 6 & 4 & 2 & 1 & 22 \\
\hline 11 & Transportation cost for feedstock & & & 1 & & & & 1 & 1 & 1 & 3 & 2 & 6 & 4 & 3 & & 22 \\
\hline 12 & Overhead cost & & & 1 & & & & 1 & & & & & 2 & 1 & 1 & & 6 \\
\hline 13 & Response to market \& policy uncertainties & & & & & & & & 1 & & 2 & 4 & 8 & 3 & 2 & & 20 \\
\hline 14 & Storage cost (for feedstock) & & & 1 & & & & 2 & 1 & & 2 & 1 & 5 & 2 & 2 & & 16 \\
\hline 15 & Storage cost (for biofuel) & & & & & & & 2 & 1 & & 1 & 1 & 4 & 1 & 2 & & 12 \\
\hline 16 & Annual investments (maintenance) & & & 1 & & & & 1 & 1 & & 1 & 2 & 6 & 3 & 1 & & 16 \\
\hline 17 & Life cycle cost with production cost & & & 1 & & & & 1 & & & & 1 & 2 & & & & 5 \\
\hline 18 & Life cycle cost without production & & & & & & & & & & 1 & 1 & 1 & 1 & 1 & & 5 \\
\hline 19 & Feedstock conversion ratio & & & & & & & & & & & & 1 & & & & 1 \\
\hline 20 & Energy saving & & 1 & & & & & 1 & 2 & & & 2 & 5 & 3 & 1 & & 15 \\
\hline 21 & Biomass availability & 1 & & & & & & & 2 & & 1 & 3 & 5 & 3 & 1 & & 16 \\
\hline 22 & Operation efficiency & 1 & & & & & & 1 & 1 & & 2 & 2 & 7 & 2 & 1 & & 17 \\
\hline 23 & Number of jobs offered & & & 1 & & 1 & 1 & 1 & & 1 & & & 6 & 1 & 1 & & 13 \\
\hline 24 & Energy taxes & & & & & & & & & 1 & & & 1 & 2 & & & 4 \\
\hline 25 & Energy policy & & & & & & & & & 1 & & & 3 & 2 & & & 6 \\
\hline 26 & Development status & & & & & & & & & 2 & & & & 1 & & & 3 \\
\hline 27 & Possible subsidies & & & & & & & & & 2 & & & & 1 & & & 3 \\
\hline 28 & Safety & & & & & & 1 & & & & 1 & 1 & & & & & 3 \\
\hline 29 & Public acceptance & & & & & & & & & & & & & 1 & & & 1 \\
\hline 30 & Food prices relation & & & & & & & & & & & & 1 & & & & 1 \\
\hline 31 & Learning curve & & 1 & & & 1 & & & & & & & & & & & 2 \\
\hline & Total observations & 7 & 6 & 12 & 0 & 5 & 7 & 15 & 21 & 9 & 26 & 39 & 110 & 64 & 30 & 2 & 353 \\
\hline
\end{tabular}


Next, stakeholders' requirements were clustered into five perspectives: Economic, environmental, technical, social, and legal. The detailed information about the five perspectives and their related requirements is shown in Figure 4.4.

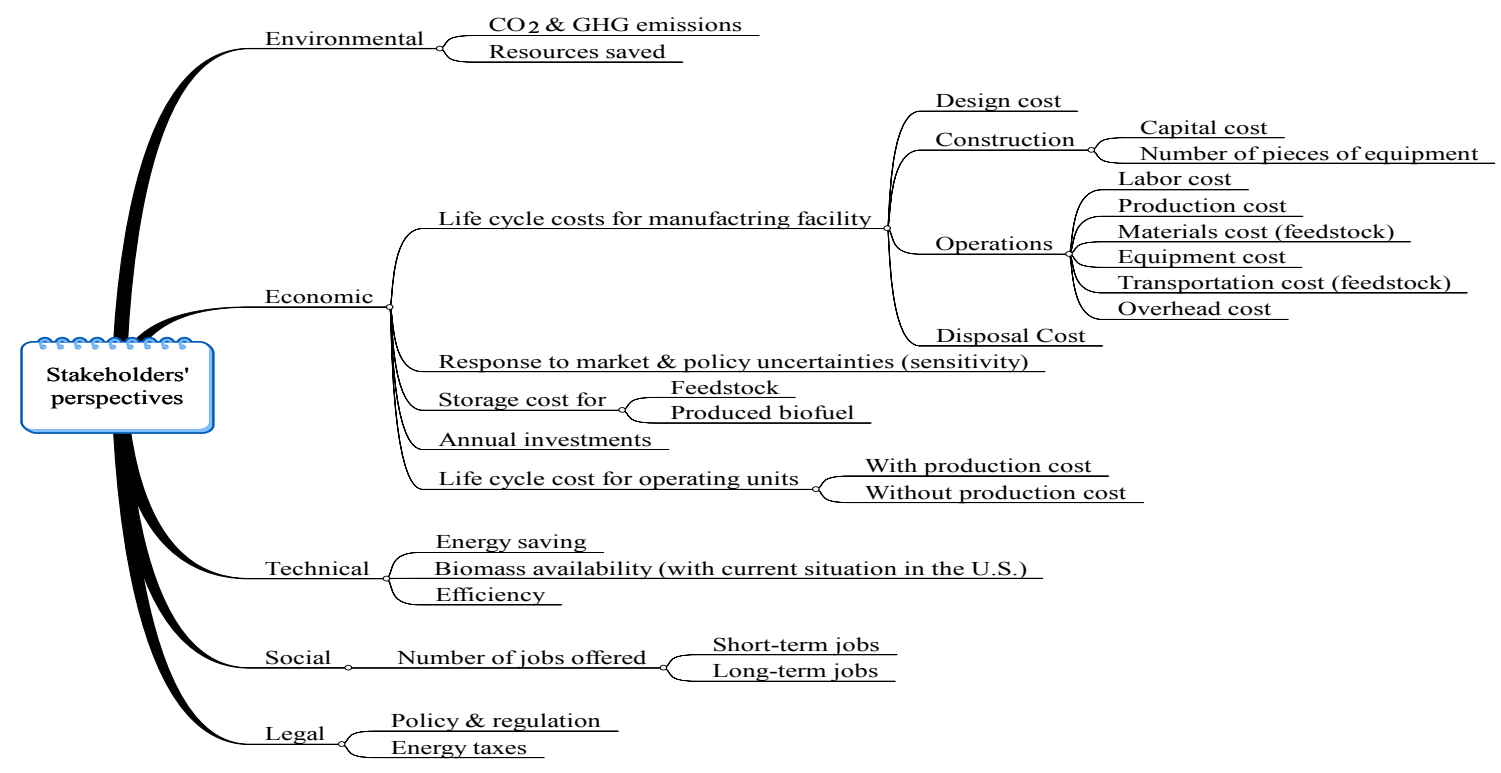

Figure 4.4. Stakeholders' perspectives

The model for this research was developed using two techniques. First, Microsoft Excel 2010 was used to create the study matrix. This matrix identifies requirements with their perspectives in the first column. Each perspective's weight is the summation of its related requirements' weights. In the first row, the three alternatives are listed from the largest to the smallest. Then, for the eighteen requirements, priorities are given based on the average of SMEs' opinions. Table 4.2 shows the final matrix (the model) using Microsoft Excel 2010. From Table 4.2, it can be observed that the largest unit's size, which is used as the base, has a total score equal to zero. Moreover, the second alternative with a capacity of 200-500 tpd has a total score equal to 3.3054. Lastly, the smallest unit's size has a total score equal to 3.9644. After normalizing the weights, the largest unit's size still has the same value where the medium one has a score equal to 0.0779 and the smallest unit's size has a score equal to 0.093 . 
Table 4.2. Final Matrix with Scores for Alternatives Analysis from the Microsoft Excel Model

\begin{tabular}{|c|c|c|c|c|c|c|c|c|c|c|c|c|c|c|c|c|}
\hline \multirow{2}{*}{$\begin{array}{l}0 \\
: \\
0 \\
0 \\
0 \\
0 \\
0 \\
0 \\
0\end{array}$} & \multirow{2}{*}{ Requirements } & \multicolumn{4}{|c|}{ Priorities } & \multirow{2}{*}{ 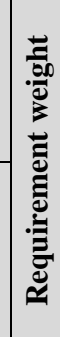 } & \multicolumn{3}{|c|}{$\begin{array}{c}\text { Alternatives ( FPU size) } \\
\text { Evaluations average } \\
\text { Pugh selection matrix: } \\
\text { Feedstock inputs (tpd) }\end{array}$} & \multicolumn{3}{|c|}{$\begin{array}{l}\text { Alternatives ( FPU size) } \\
\text { Weighted scores } \\
\text { Pugh selection matrix: } \\
\text { Feedstock inputs (tpd) }\end{array}$} & \multirow{2}{*}{ 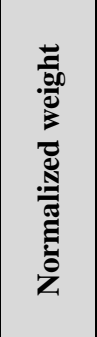 } & \multicolumn{3}{|c|}{$\begin{array}{l}\text { Alternatives ( FPU size) } \\
\text { Normalized scores } \\
\text { Pugh selection matrix: } \\
\text { Feedstock inputs (tpd) }\end{array}$} \\
\hline & & $\mathrm{H}$ & M & $\mathrm{L}$ & $\begin{array}{l}\dot{\overline{0}} \\
\vdots \\
\vdots \\
\vdots \\
0 \\
0\end{array}$ & & 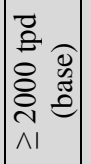 & $\begin{array}{l}8 \\
\qquad \\
1 \\
8 \\
1\end{array}$ & 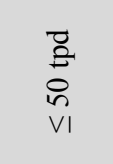 & 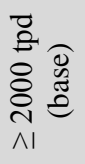 & 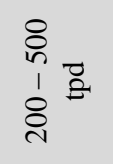 & $\begin{array}{l}\vec{Z} \\
\text { E } \\
0 \\
\text { in } \\
\text { VI }\end{array}$ & & 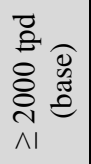 & $\begin{array}{l}8 \\
i n \\
1 \\
8 \\
8 \\
0\end{array}$ & $\begin{array}{l}\text { Dृ } \\
\text { E } \\
i n \\
\text { VI }\end{array}$ \\
\hline \multirow{2}{*}{ 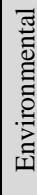 } & $\mathrm{CO}_{2}$ emission & 4 & 4 & 2 & 0 & 2.2 & 0 & 0.111 & -0.444 & 0 & 0.244 & -0.978 & 0.052 & 0 & 0.0058 & -0.0230 \\
\hline & Resources saved & 5 & 4 & 1 & 0 & 2.4 & 0 & 0.125 & -0.125 & 0 & 0.300 & -0.300 & 0.057 & 0 & 0.0071 & -0.0071 \\
\hline \multirow{11}{*}{ 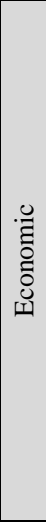 } & Design cost & 4 & 4 & 2 & 0 & 2.2 & 0 & 0.333 & 0.778 & 0 & 0.733 & 1.711 & 0.052 & 0 & 0.0173 & 0.0403 \\
\hline & Capital cost (including Equipment) & 9 & 1 & 0 & 0 & 2.9 & 0 & -0.700 & -1.300 & 0 & -2.030 & -3.770 & 0.068 & 0 & -0.0478 & -0.0888 \\
\hline & Equipment cost & 7 & 2 & 1 & 0 & 2.6 & 0 & -0.500 & -1.200 & 0 & -1.300 & -3.120 & 0.061 & 0 & -0.0306 & -0.0735 \\
\hline & Labor cost & 2 & 2 & 6 & 0 & 1.6 & 0 & -0.700 & -1.100 & 0 & -1.120 & -1.760 & 0.038 & 0 & -0.0264 & -0.0415 \\
\hline & Production cost in the facility & 7 & 3 & 0 & 0 & 2.7 & 0 & -0.444 & -0.889 & 0 & -1.200 & -2.400 & 0.064 & 0 & -0.0283 & -0.0566 \\
\hline & \begin{tabular}{|l|} 
Materials cost (feedstock) \\
\end{tabular} & 7 & 3 & 0 & 0 & 2.7 & 0 & 0.400 & 0.700 & 0 & 1.080 & 1.890 & 0.064 & 0 & 0.0255 & 0.0445 \\
\hline & Transportation cost for feedstock & 5 & 2 & 2 & 1 & 2.3 & 0 & 1.111 & 2.000 & 0 & 2.593 & 4.667 & 0.055 & 0 & 0.0611 & 0.1100 \\
\hline & $\begin{array}{l}\text { Response to market \& policy } \\
\text { uncertainties }\end{array}$ & 7 & 2 & 1 & 0 & 2.6 & 0 & 0.556 & 0.889 & 0 & 1.444 & 2.311 & 0.061 & 0 & 0.0340 & 0.0545 \\
\hline & Storage cost for feedstock & 3 & 5 & 2 & 0 & 2.1 & 0 & 0.500 & 0.900 & 0 & 1.050 & 1.890 & 0.049 & 0 & 0.0247 & 0.0445 \\
\hline & Storage cost for produced biofuel & 4 & 1 & 4 & 1 & 2.0 & 0 & -0.222 & -0.333 & 0 & -0.444 & -0.667 & 0.047 & 0 & -0.0105 & -0.0157 \\
\hline & Annual investments (maintenance) & 6 & 4 & 0 & 0 & 2.6 & 0 & 0.300 & 0.100 & 0 & 0.780 & 0.260 & 0.061 & 0 & 0.0184 & 0.0061 \\
\hline \multirow{3}{*}{ 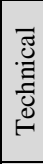 } & Energy saving & 3 & 6 & 1 & 0 & 2.2 & 0 & -0.500 & -0.700 & 0 & -1.100 & -1.540 & 0.052 & 0 & -0.0259 & -0.0363 \\
\hline & \begin{tabular}{|l|}
$\begin{array}{l}\text { Biomass availability } \\
\text { (with current situation in the U.S.) }\end{array}$ \\
\end{tabular} & 6 & 3 & 1 & 0 & 2.5 & 0 & 0.800 & 1.400 & 0 & 2.000 & 3.500 & 0.059 & 0 & 0.0471 & 0.0825 \\
\hline & Operation efficiency & 4 & 6 & 0 & 0 & 2.4 & 0 & -0.500 & -0.600 & 0 & -1.200 & -1.440 & 0.057 & 0 & -0.0283 & -0.0339 \\
\hline 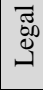 & Policy \& regulations & 6 & 4 & 0 & 0 & 2.6 & 0 & -0.125 & 0.250 & 0 & -0.325 & 0.650 & 0.061 & 0 & -0.0077 & 0.0153 \\
\hline $\begin{array}{l}\cdot \bar{\pi} \\
0 \\
\infty\end{array}$ & Number of jobs offered & 2 & 4 & 4 & 0 & 1.8 & 0 & 1.000 & 1.700 & 0 & 1.800 & 3.060 & 0.042 & 0 & 0.0424 & 0.0721 \\
\hline & & & & & & & \multicolumn{3}{|r|}{ bres: } & 0 & 3.305 & 3.964 & 1.000 & 0 & 0.0779 & 0.0934 \\
\hline
\end{tabular}

The second model was created using Logical Decisions V7.1. In this software, the overall goal and its sub-objectives, which are the five perspectives, are listed with all related requirements as measures for each sub-objective. Then, all of the weights are assigned by using the direct entry function in the software. Figure 4.5 presents the final hierarchy with the assigned weights. 


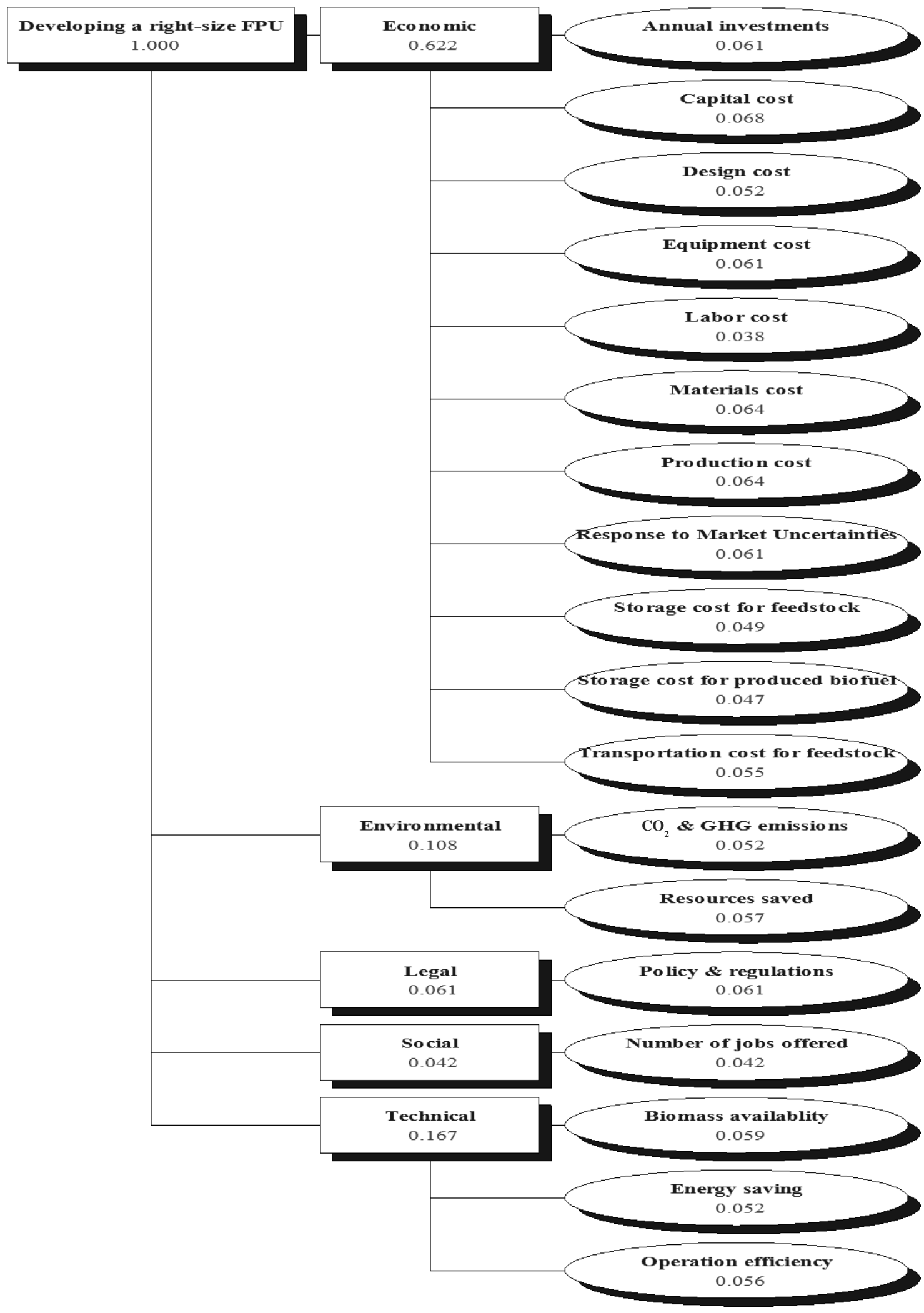

Figure 4.5. Final hierarchy with assigned weights using Logical Decisions ${ }^{\circledR}$ V7.1 
The model illustrated in Figure 4.5 was analyzed. As a result, the last alternative, which is the facility size with a capacity of $50 \mathrm{tpd}$, is the best selection, with a utility value equal to 0.523. This result matches the one from the first Excel model. Likewise, the medium facility size (200-500 tpd facility) is the second best alternative. This result is also compatible with the one from the first Excel model with a close utility value to the other alternative, 0.519. Figure 4.6 shows the alternatives ranking and utility values for the model from Logical Decisions V7.1.
Alternative Utility
1.50 tpd
0.523
2. $200-500$ tpd
0.519
3. 2000 tpd
0.500

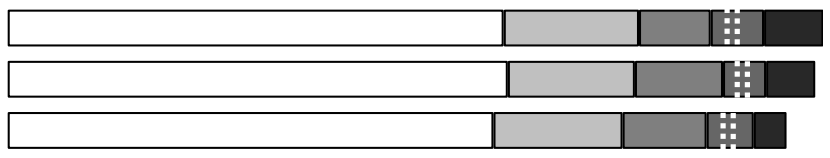
$\square$ Economical
it. Legal
Technical
Social
$\square$ Environmental Impact

Figure 4.6. Final utilities for the alternatives analysis from the model of Logical Decisions ${ }^{\circledR}$

Thus, analysis of both models provides the same results. Consequently, both models can be considered as two different multi-criteria decision analysis tools to evaluate FPUs for energy production.

By analyzing the additional perspectives and their related factors listed in the interview form, as illustrated in Figure 4.7, the researchers found that:

- Almost all the SMEs interviewed believed in the importance of manufacturing process improvement in the decision-making process. An SME from academia argued that the absence of this perspective in previous research is due to the lack of such units' manufacturing in addition to the novelty of these units' appearance in the area even given its importance.

- Almost all the SMEs interviewed believed in the importance of considering the safety perspective in such a decision. It is a very important factor to be considered in decisions of manufacturing or building and operating FPUs. 
In addition, SMEs believe that some other important requirements should be considered in this decision. Those potential requirements also clustered into the five perspectives. From an economic perspective, the compatibility of FPUs with the current infrastructure in the Midwest region of the U.S. has not received attention in previous studies. In addition, an SME mentioned that there is not much analysis about this requirement in the FPUs' area, to his knowledge. However, $60 \%$ of SMEs evaluated the compatibility of FPUs with current infrastructure as a highly important requirement while the rest evaluated it as having medium importance to the decision. Figure 4.7 shows the detailed evaluation of the potential perspectives based on the requirements evaluation performed by the SMEs. 


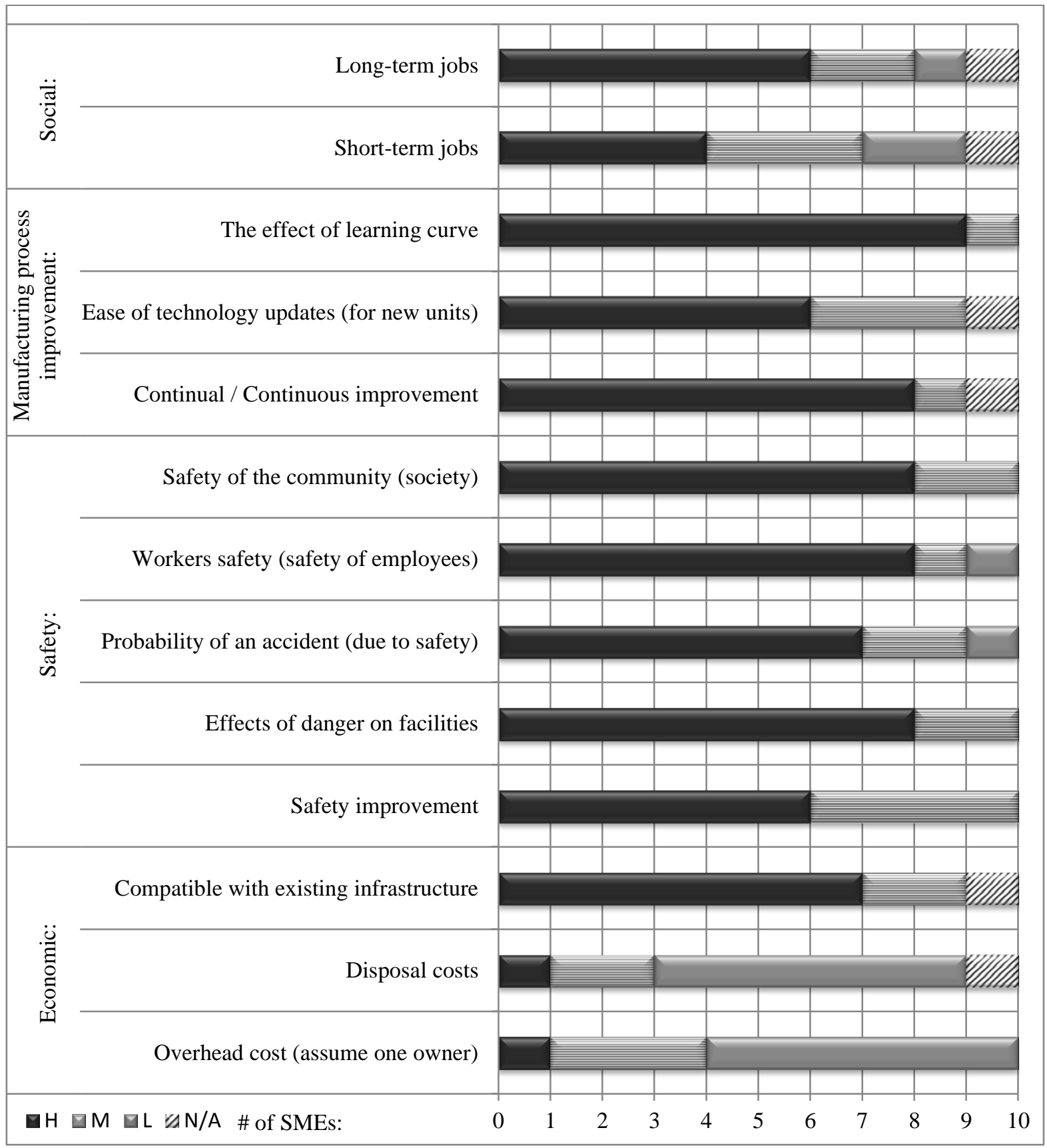

Figure 4.7. Potential perspectives' importance

Sensitivity Analysis

A sensitivity analysis was conducted on the overall perspectives using Logical Decisions V7.1. When a sensitivity analysis was performed on an individual perspective, the weight given 
to that perspective was adjusted up or down. On the other hand, the weights given to the other perspectives were adjusted proportionally. Because the economic perspective was given the highest priority by the SMEs, a sensitivity analysis was conducted on this perspective first (see Figure 4.8). The economic perspective weight was varied from 0 to $100 \%$. The sensitivity analysis showed that, when the economic perspective was weighted at any value from 0 to $79 \%$, the smallest FPU (50 tpd) was recommended. When the weight given to the economic perspective exceeded 79\%, the medium-sized FPU (200-500 tpd) was recommended. The vertical line at $62 \%$ was the weight given to the economic perspective in the initial analysis, and at this point, the smaller FPUs is recommended.

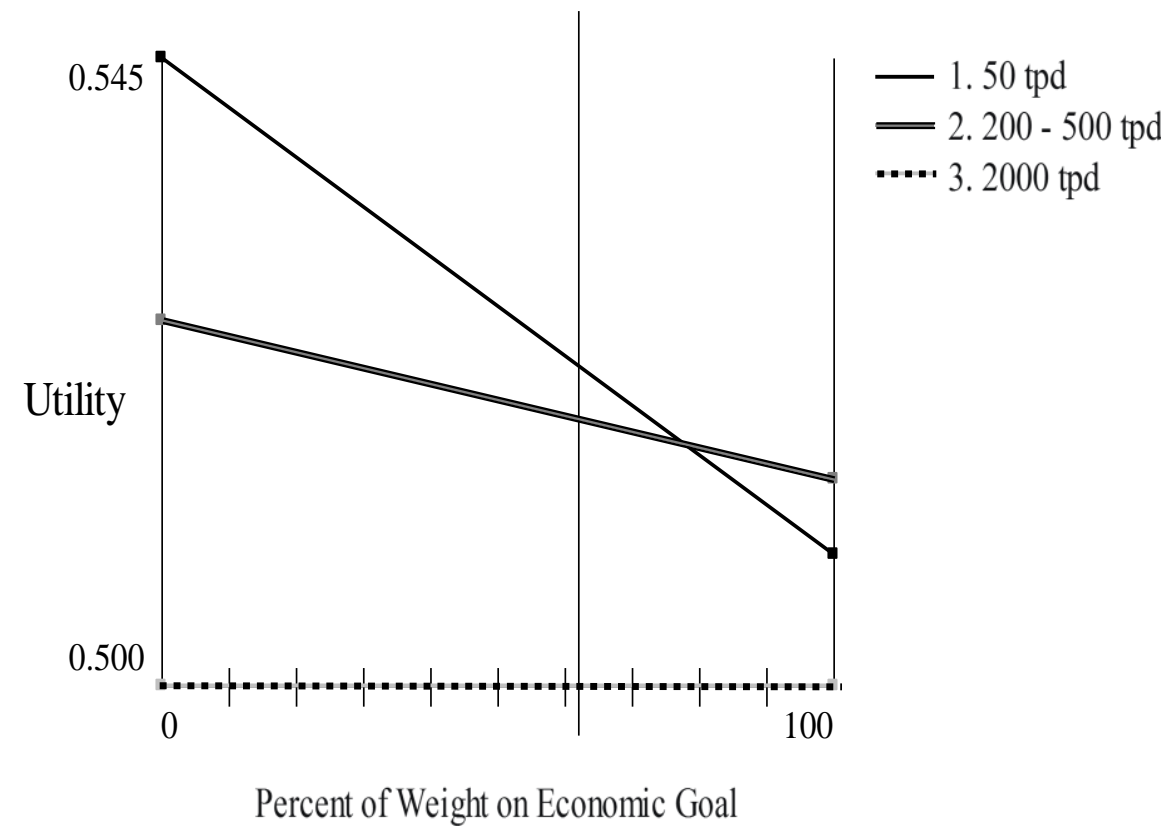

Figure 4.8. Sensitivity analysis on the economic perspective

Some SMEs believe that the economic perspective is the most important factor. Moreover, they mentioned that everything in such a decision should be converted to dollar equivalents regardless of the original perspectives and because businesses usually only focus on making profit. 
The same analysis was conducted on the environmental, technical, social, and legal perspectives to examine the robustness of recommending the small FPU (Figure 4.9). It is interesting to note that the small FPU is recommended in $35(70 \%)$ of the 50 sensitivity analysis cases run. When technical, social, or legal perspectives were weighted from $10 \%$ to $100 \%$, the smallest FPU was recommended. An increase in the weight of the environmental perspective resulted in the medium FPU becoming the recommended alternative when the weight increases by $10 \%$ or more. A summary of all perspectives' weights, and their impacts on the recommended alternative, is shown in Figure 4.9.

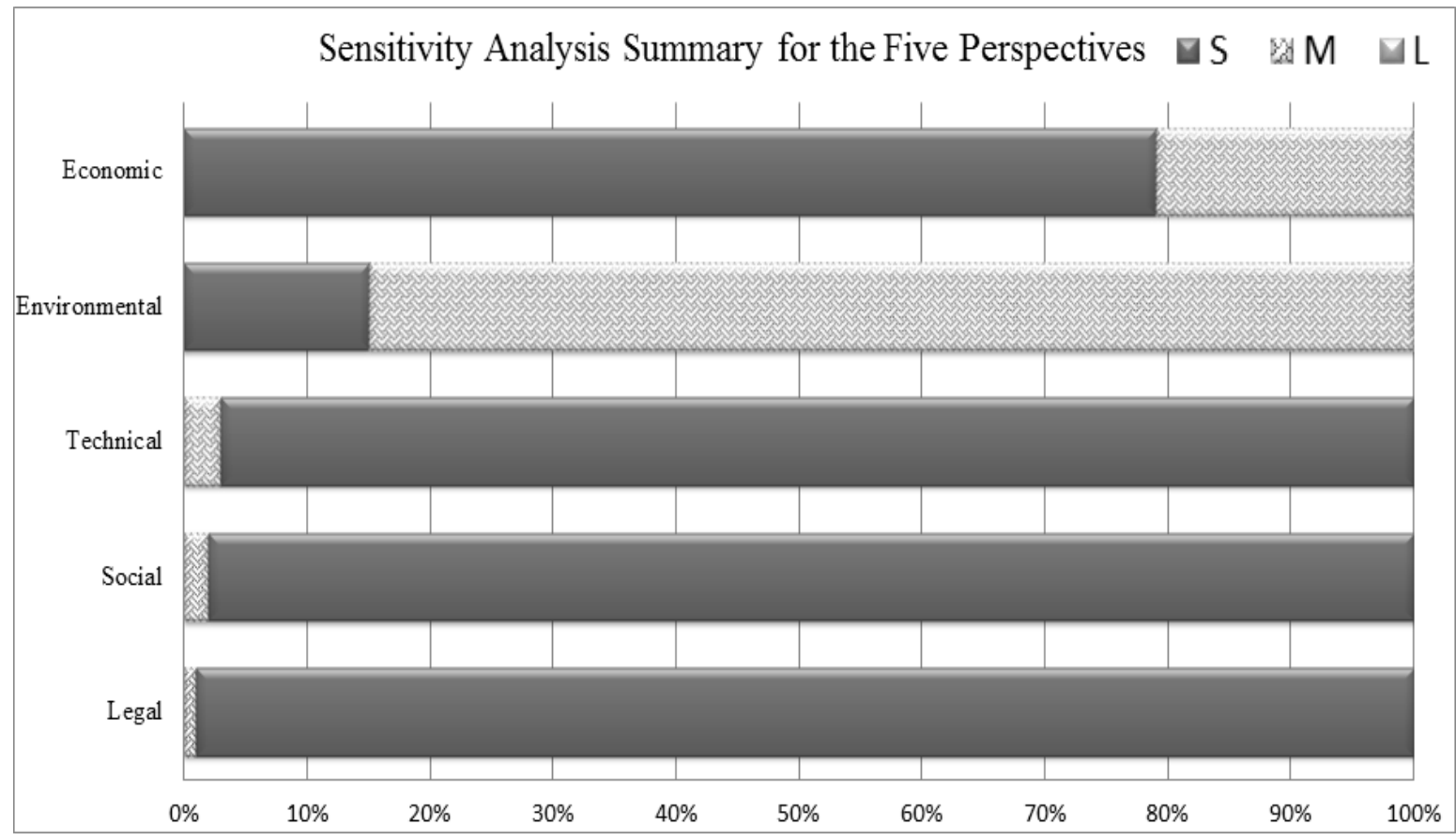

Figure 4.9. Sensitivity analysis summary for the five perspectives

Due to the strong exchange of roles between the smallest FPU alternative and the medium FPU alternative, the changing priorities, and the close utility values for both of them, the relationship between these two alternatives was investigated more on the perspectives level using a tornado chart as shown in Figure 4.10. 


\begin{tabular}{lrl|r} 
Developing a right-size FPU Goal Utility for & $\begin{array}{l}1.50 \mathrm{tpd} \\
2.200-500 \mathrm{tpd} \\
\end{array}$ & $\begin{array}{l}\text { Total Difference } \\
\end{array}$ & $\frac{0.519}{0.004}$ \\
& Difference & $2.200-500 \mathrm{tpd}$ & $1.50 \mathrm{tpd}$ \\
\hline Total Difference & 0.004 & & \\
Environmental & -0.011 & & \\
Social & 0.007 & & \\
Legal & 0.006 & & \\
Technical & 0.005 & & \\
Economic & -0.003 & &
\end{tabular}

Figure 4.10. Sensitivity analysis on best two FPU sizes

Conclusions

Engineering managers are often faced with complex decisions where there are multiple stakeholders, and often conflicting sets of requirements. This research addressed a sample from this class of problems by using a MAUT process with the integration of a Pugh concept selection matrix to determine the appropriate production unit size to satisfy all stakeholders in a biofuel industry example. MAUT and the Pugh concept selection matrix enabled the development of a model that represented the feasible trade space and allowed decision makers to fully explore that trade space to determine the performance of each alternative.

The process started by taking the eighteen requirements expressed by the stakeholders and organizing them into five perspectives: 1) Economic, 2) environmental, 3) legal, 4) social, and 5) technical. The recommended FPU size was found to be dependent on these decision maker perspectives; the smaller FPU was recommended from the economic, technical, social, and legal perspectives, while the medium FPU was recommended from the environmental perspective. This finding is consistent with prior research that has noted differences in 
requirements between economic and environmental perspectives, and work which has noted that smaller-scale technologies have not been proven to be the right solution in all situations.

SMEs in this study were used to help clarify requirements and perspectives; however, they too had different views on the relative value of different FPUs. The decision-making process presented helped clarify specific reasons for these conflicting values. Overall, the smaller FPU was generally recommended and the medium FPU was the second best alternative. Just as the recommendation changed depending on perspectives and priorities in the analysis, the SMEs also showed differences in opinions.

The decision-making process used helped frame the trade space. It provided insight to help decision makers understand the problem and make recommendations. The use of a range of SMEs from different stakeholder groups clarified differences in perspectives and how each stakeholder viewed the problem. For example, the authors noticed that industrial SMEs focused more on the economic side and converted all their perspectives to equivalent dollars in their discussions. This observation is consistent with the results of a previous study that indicated that business personnel and private sector personnel tend to highlight the importance of profit and the financial side more in their evaluations (Michalopoulos, Landeweerd, Werf-Kulichova, Puylaert, \& Osseweijer, 2011).

Two of the SMEs from the industrial sector believed that the medium size is the best alternative and that by going to the smallest FPUs, the advantages of scale will be lost. One SME pointed out that such a decision is not linear. Instead of considering which size fits the requirements best, stakeholders should decide to start manufacturing the smallest unit size and then duplicate it or increase the size as needed. Therefore, the first unit would serve as an 
experimental unit to examine the situation and improve the learning curve since biofuel manufacturing is a new field.

Many engineering managers often rely on a small set of decision-making tools because they are familiar with them and have had success using them in the past. Unfortunately, they may try to use this tool to address any decision-making problem regardless of its suitability for the problem at hand (Componation \& Nicholls, 2011). This research presents the power of using an integrated methodology for decision-making which is one of the recent common methods for solving complex decision problems (Ho, Xu, \& Dey, 2010; Govindan, Rajendran, Sarkis, \& Murugesan, 2013).

The integration between the Pugh method and MAUT was proposed and utilized in this research as a powerful integrated methodology approach for solving a complex decision problem. Moreover, for such big decisions, using quantitative and qualitative data helps build the needed model for a decision.

The authors noticed that the SMEs did not have a consistent evaluation for most of the requirements. Therefore, SME group size and diversity could have an influence on the final decision for this study. Their impact on the results sensitivity could be examined as future work.

This study can be replicated also by extending the number of SMEs. More sensitivity analysis could be done on the requirements level, to examine their impact on selecting optimal size FPUs. Due to the strong exchange of roles between the smallest unit size alternative and the medium unit size alternative, as well as on the changing priorities and the close utility values for both of them, the relationship between these two alternatives should be investigated more at the requirements level in future work. 
Further examination of the requirements that comprise the environmental perspective is needed to illustrate why the recommendation changes as environmental weight is increased. Moreover, because of the importance of this kind of decision, each one of the high priority requirements should be studied deeply using the same analysis conducted on previous perspectives.

SMEs believe there are other requirements that should be included in the FPUs' manufacturing not mentioned previously in the literature or in the potential requirements. Those requirements could be investigated and classified according to the existing perspectives in future work as well. Thus, this study could be replicated by considering the potential perspectives, which were classified as important ones according to the SMEs' view. Future work could also investigate the similarities and differences between the academic experts and the industrial (private sector) experts to determine if they focus on similar perspectives. Finally, future work could generalize the methodology used to other forms of renewable energy manufacturing plants. 


\title{
CHAPTER 5. STAKEHOLDERS' REQUIREMENTS ASSESSMENT FOR BIOFUEL PRODUCTION
}

\author{
A paper submitted to Energy Journal
}

Mostafa F. Fawzy, Paul J. Componation, and Guiping Hu

\begin{abstract}
Biofuel is one of the best alternatives for fossil fuel in the United States and other countries. Increasing attention has been attracted to biofuel production process. Fast pyrolysis as one of the most promises thermochemical based advanced biofuel production techniques has been brought to the forefront of industry. With limited information about the manufacturing of fast pyrolysis units, a better understanding of stakeholders' requirements is timely and necessary. This study develops a comprehensive analysis of stakeholders' requirements. Individual subject matter experts were asked to rank, review, and evaluate a set of requirements for a unity of fast pyrolysis unit sizes. The requirements with their evaluation were then used to determine the most effective fast pyrolysis unit size. The analysis showed that the smallest unit size that is able to process an average of 50 tons per day is the best alternative based on $50 \%$ of the high-prioritized requirements. However, when viewed from $37.5 \%$ of the high-prioritized requirements, a unit able to process at least 2000 tons per day range is the best alternative. Moreover, this work provides detailed discussions on the multiple requirements, including a comparison between the two fast pyrolysis units with the highest utilities.
\end{abstract}


Keywords: Decision-making, Biofuel, Pyrolysis, Fast pyrolysis unit

\section{Introduction}

Renewable energy has been gaining attention globally. In the U.S., renewable energy production started in 1973 (EIA, 2001) and by 2011, 8\% of the U.S. energy production came from renewable sources. This percentage increased to $9 \%$ in 2012 and then to $11.4 \%$ by 2013 (EIA, 2014). In 2013, 9.298 out of 81.669 quadrillion British thermal units came from renewable sources (EIA, 2014). Renewable energy could be used in transportation, industry, residence, commerce, and/or electric power consumptions (EIA, 2014). Approximately half of the renewable energy production is from biomass (EIA, 2014).

Biofuel production has a large number of stakeholders. Each stakeholder has different perspective and requirements (Fawzy \& Componation, 2014). These different requirements can make the selection of a biofuel production strategy a challenge. As a result, this research objective, optimizing the production of a Fast Pyrolysis Unit (FPU), is classified as a complex decision problem that can be solved using a Multi-Attribute Utility Theory (MAUT) approach (Min, 1994). The MAUT application follows the same pattern of normal decision-making approaches (Dyer, 2005). Decision makers often have to make decisions with limited information, especially in new areas such as advanced biofuel production. In order to design valid decision-making support systems to assist decision makers in these situations, a detailed analysis on the available information is required. This should also include investigation for factors that influence the decision under different conditions and situations. This research explains the requirements-level analysis and its sensitivity analysis.

In this research, three alternatives FPUs sizes are analyzed. The first alternative is small FPUs with a capacity of 50 tons per day (tpd). The second alternative is a medium FPUs with a 
capacity between 200 to 500 tpd. Finally, the third alternative is the biggest FPUs with a capacity 2000 tpd or more.

A decision-making model was developed using Logical Decision software V7.2 to investigate the best FPUs' size based on stakeholders' requirement.

This study aims to investigate the impact of stakeholders' requirements. This research also included a sensitivity analysis to determine the impact of high-prioritized requirements on FPU selection.

This research supports further development of an approach for the manufacturing, placement, and right sizing of FPUs for biofuel production infrastructure. Decision-makers face a lot of open-ended problems such as deciding FPUs size for biofuel production in real life. This problem is classified as an open-ended problem because it could have several correct answers based on different stakeholders' perspectives and requirements. Further, authors believe that the presence of additional information in the future may influence the decision and help better understand the problem. This may change the current results or decision based on the conceptual assessment of FPUs' sizes.

This research provides significant insights into complex decision problems with the use of powerful tool. In addition, the use of the Logical Decisions tool for the sensitivity and robustness analysis, as an integrated tool, developed better understanding of decision-making process. Moreover, this research presents an example of a decision-making approach that uses quantitative and qualitative criteria. However, a variety of methodologies, tools and techniques could be adopted to solve the problem. The suggested approach could be applied in other decision-making processes for other renewable energy resources with lack of information and multi-stakeholders involvement for selecting the best option from several alternatives. 


\section{Literature Review}

FPU's size selection is considered as a complex decision problem due to a verity of stakeholders, multi-alternatives, and multi-requirements involved in the decision-making process. It is illustrated in the literature that there are multiple BPS groups affecting the decisionmaking process each with a specific typology and perspective (Youngs, 2012; Fawzy \& Componation, 2014). Thirty-six groups are identified then classified into eight typology sets as the Biofuel Production Stakeholders (BPSs), which are involved in FPU's manufacturing as shown in Figure 5.1 (Fawzy \& Componation, 2014). That identification can classification of BPSs was done by applying the theory of stakeholder identification, which initiated be Freeman (1984).

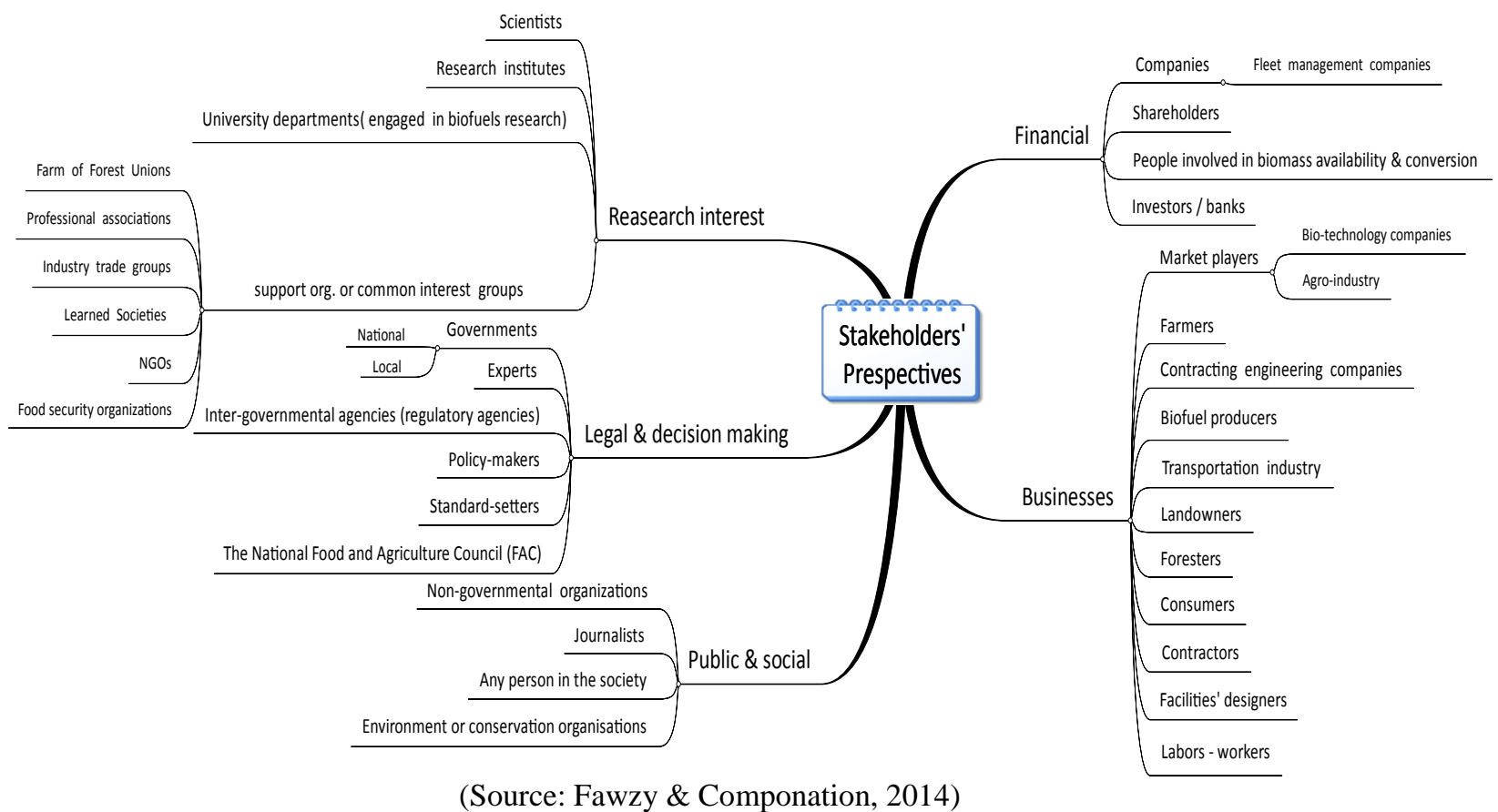

Figure 5.1. Mind map diagram for BPS perspectives and groups

Research studies published between 1996 and 2014 that mention biofuel manufacturing requirements were reviewed. In addition, a list of 31 requirements presented in a study done by 
Fawzy \& Componation (2015) as shown in Table 5.1 is being considered as the base of this research requirements recognition.

Table 5.1. References for Stakeholders' Requirements for Biofuel Production (Fawzy \& Componation, 2015)

\begin{tabular}{|c|c|c|c|c|c|c|c|c|c|c|c|c|c|c|c|c|c|}
\hline \# & Requirement $\quad$ Year & ๙ั & ) & ำ & ஜి & ণั & ஜุ & ๖ి & ิิ) & 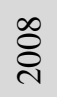 & ஓ̊ & $\stackrel{0}{\circ}$ & $\overline{\check{c}}$ & $\stackrel{\sim}{\stackrel{2}{\circ}}$ & $\frac{m}{\tilde{\nu}}$ & 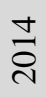 & $\stackrel{\overparen{\Xi}}{0}$ \\
\hline 1 & $\mathrm{CO}_{2} \& \mathrm{GHG}$ emissions & 1 & 1 & 1 & & & 1 & 2 & 1 & 1 & 3 & 4 & 7 & 4 & 2 & & 28 \\
\hline 2 & Land use exchange & 1 & & & & & 1 & & & & & 1 & 2 & 1 & 1 & & 7 \\
\hline 3 & Resources saved & 1 & & & & & 1 & 1 & 1 & & & 4 & 5 & 3 & 1 & & 17 \\
\hline 4 & Design cost & & & & & & & & 1 & & & 1 & 2 & 3 & & & 7 \\
\hline 5 & Capital cost & 1 & 1 & 1 & & 1 & & & 2 & & 1 & 2 & 6 & 4 & 1 & & 20 \\
\hline 6 & Number of pieces of equipment & & & & & & & & & & & & 1 & 2 & 1 & & 4 \\
\hline 7 & Equipment cost & & & 1 & & & & & & & 2 & 2 & 3 & 3 & 2 & & 13 \\
\hline 8 & Labor cost & & 1 & 1 & & 1 & & & 1 & & & 1 & 6 & 5 & 2 & & 18 \\
\hline 9 & Production / Operation cost & 1 & 1 & 1 & & 1 & 1 & 1 & 3 & & 4 & 1 & 9 & 4 & 2 & 1 & 30 \\
\hline 10 & Materials cost (feedstock) & & & 1 & & & 1 & & 2 & & 2 & 3 & 6 & 4 & 2 & 1 & 22 \\
\hline 11 & Transportation cost for feedstock & & & 1 & & & & 1 & 1 & 1 & 3 & 2 & 6 & 4 & 3 & & 22 \\
\hline 12 & Overhead cost & & & 1 & & & & 1 & & & & & 2 & 1 & 1 & & 6 \\
\hline 13 & Response to market \& policy uncertainties & & & & & & & & 1 & & 2 & 4 & 8 & 3 & 2 & & 20 \\
\hline 14 & Storage cost (for feedstock) & & & 1 & & & & 2 & 1 & & 2 & 1 & 5 & 2 & 2 & & 16 \\
\hline 15 & Storage cost (for biofuel) & & & & & & & 2 & 1 & & 1 & 1 & 4 & 1 & 2 & & 12 \\
\hline 16 & Annual investments (maintenance) & & & 1 & & & & 1 & 1 & & 1 & 2 & 6 & 3 & 1 & & 16 \\
\hline 17 & Life cycle cost with production cost & & & 1 & & & & 1 & & & & 1 & 2 & & & & 5 \\
\hline 18 & Life cycle cost without production & & & & & & & & & & 1 & 1 & 1 & 1 & 1 & & 5 \\
\hline 19 & Feedstock conversion ratio & & & & & & & & & & & & 1 & & & & 1 \\
\hline 20 & Energy saving & & 1 & & & & & 1 & 2 & & & 2 & 5 & 3 & 1 & & 15 \\
\hline 21 & Biomass availability & 1 & & & & & & & 2 & & 1 & 3 & 5 & 3 & 1 & & 16 \\
\hline 22 & Operation efficiency & 1 & & & & & & 1 & 1 & & 2 & 2 & 7 & 2 & 1 & & 17 \\
\hline 23 & Number of jobs offered & & & 1 & & 1 & 1 & 1 & & 1 & & & 6 & 1 & 1 & & 13 \\
\hline 24 & Energy taxes & & & & & & & & & 1 & & & 1 & 2 & & & 4 \\
\hline 25 & Energy policy & & & & & & & & & 1 & & & 3 & 2 & & & 6 \\
\hline 26 & Development status & & & & & & & & & 2 & & & & 1 & & & 3 \\
\hline 27 & Possible subsidies & & & & & & & & & 2 & & & & 1 & & & 3 \\
\hline 28 & Safety & & & & & & 1 & & & & 1 & 1 & & & & & 3 \\
\hline 29 & Public acceptance & & & & & & & & & & & & & 1 & & & 1 \\
\hline 30 & Food prices relation & & & & & & & & & & & & 1 & & & & 1 \\
\hline 31 & Learning curve & & 1 & & & 1 & & & & & & & & & & & 2 \\
\hline & Total observations & 7 & 6 & 12 & 0 & 5 & 7 & 15 & 21 & 9 & 26 & 39 & 110 & 64 & 30 & 2 & 353 \\
\hline
\end{tabular}

From a total of 353 observations noted in the literature for the past two decades, both requirements of capital cost and response to market and policy uncertainties (flexibility) were 
mentioned 20 times $(5.7 \%)$. A total of $30(8.5 \%)$ observations were also stated the production cost requirement. Moreover, both annual investments (maintenance) and biomass availability requirements are mentioned 16 times $(4.5 \%)$ in the previous research on biofuel production. Material cost requirement is observed 22 times $(6.2 \%)$ in those previous research where equipment cost is mentioned 13 times (3.7\%). Finally, energy policy requirement was mentioned 6 times $(1.7 \%)$.

It is important to make biofuels a feasible option to increase energy production, as well as national and environmental securities by improving sustainability and delivering low cost competitive products to the end-user market (Awudu \& Zhang, 2012). However, little work has been done to understand what the optimal size of biomass processing facility itself (Larasati, Liu, \& Epplin, 2012).

High capital cost is one of the major barriers to investing in advanced bio-refineries. Biorefineries share many similarities with fossil-fuel refineries including the need for significant financing. Fossil-fuel refineries reduce their capital costs through large-scale deployments that take advantage of economies of scale. Crude oil refineries in the United States produce an average of 126,000 barrels per day (bpd), and the largest U.S. refinery can process over 560,000 bpd (3\% of the total U.S. refining capacity) (Wright \& Brown, 2007). A review of other studies shows that bio-refineries capacities are often in the order of $10,000 \mathrm{bpd}$. This is smaller than traditional fossil fuel refineries due in a large part because of the logistical challenges faced by biomass supply chains that negate savings from economies of scale (Richard, 2010). Biorefineries also need to reduce costs even at small-scale but there is no clear path on how to do this. 
Industry stakeholders usually support biofuel development when there is an opportunity for profit (Michalopoulos, Landeweerd, Werf-Kulichova, Puylaert, \& Osseweijer, 2011). However, more work is recommended to decide the best unit size of biomass processing (Dwivedi \& Alavalapati, 2009; Larasati, Liu, \& Epplin, 2012; Fawzy \& Componation, 2015), while researchers noticed that the optimal size of a biofuel unit or facility depends on many variables such as capital, operation, transportation, and raw material costs (Wright \& Brown, 2007; Larasati, Liu, \& Epplin, 2012; Fawzy \& Componation, 2015).

The challenge of developing a distributed bio-refinery system is to overcome the conventional cost models that predict the unit cost of a production will decrease as the facility size becomes larger (Arrow, 1962; McDonald \& Schrattenholzer, 2001; Tsuchiya \& Kobayashi, 2004). The concept of "economies of scale" exemplified that there is a positive linear relationship between the size of a facility and the production output. On contrast, the relationship between the size of a facility and the construction, operations, and/or maintenance costs is not linear. The concept does not take into account recent developments in advanced manufacturing technologies. Some of these new manufacturing approaches as well as some new production strategies proposes that bigger may not be better. Big facilities may cost more than smaller ones (Jack, 2009). Some industries, such as aerospace, have begun to explore other design approaches to move away from the bigger is better approach (Componation \& Collopy, 2012). Attention is now being paid to non-technical parameters that can drive costs in developing new systems (Hamaker \& Componation, 2010). In the case of Biofuel Production, the result is complicated because of the transportation costs of distributed and low-density materials. According to Wright \& Brown (2007), there is an optimal plant size for the lowest unit cost of biobased production. 
Nevertheless, this optimal size is often so large that capital investment for advanced biorefineries is estimated to be as much as a billion dollars.

BPSs’ perspectives and requirements are identified in Fawzy and Componation study (2015). From that work, ten subject matter experts (SMEs) evaluated a set of eighteen requirements as the most commonly noted requirements for biofuel units manufacturing. From that study, a detailed analysis of the BPSs' requirements is recommended as a future work. In this research, the set of the identified eighteen requirements is used for the stakeholders' requirements assessment for FPUs size selection.

The analysis of three different size FPUs is considered here. First unit's size is selected to be the unit that consumes $2000 \mathrm{tpd}$. Current petroleum refineries produce more than 10,000 tpd and small corn ethanol plants consume 2,000 tpd of biomass (Wright, Brown, \& Boateng, 2008). This large facility size has been determined to be the optimal size for a Midwestern biorefinery based on typical biomass yields and farm participation. Many biofuel papers use 2000 tpd as their base case size to allow for easy comparison with previous studies even though it is not confirmed as the optimal size (Wright, Brown, \& Boateng, 2008; Anex, et al., 2010; Swanson, Platon, Satrio, \& Brown, 2010; Wright, Daugaard, Satria, \& Brown, 2010; Wright, personal communication, May 14, 2013).

Second unit's size is the unit which consumes 200 or up to 500 tpd of feedstock, and that is a size that researchers have envisioned that a small group of farmers would invest in (Ringer, Putsche, \& Scahill, 2006; Wright, Brown, \& Boateng, 2008; Wright, Daugaard, Satria, \& Brown, 2010). The capacity and costs for these sizes are comparable to the early ethanol and biodiesel plants (Wright, personal communication, May 14, 2013). 
The third unit's size is the smallest unit that consumes 50 tpd of biomass. It is about the largest feasible size for a 'mobile' unit that companies (Dynamotive \& Ensyn) try to produce. In a study done in 2004, it stated, "Fast pyrolysis has proven itself to be a technically viable technology for the 0 to 45 tpd plant size range (LaClaire, Barrett, \& Hall, 2004).

Most of the current research on biofuel production as a renewable energy resource has been done in Europe (Dwivedi \& Alavalapati, 2009). On the other hand, biofuel production has not been as heavily researched as other components of the renewable energy industry in the U.S. Selecting the right size for the biofuel production facility has become more important as production increases in the U.S. In order to design a viable biofuel production system, further analysis is needed. Therefore, this research fills a gap in our knowledge about the optimal size of biofuel production facilities by providing a detailed explanation of the requirements level analysis in addition to the sensitivity analysis of the high-prioritized requirement for this complex decision-making problem.

This research right-sizing FPUs using a Multi-Criteria Decision Analysis (MCDA) as a decision-making approach. This will lead to developing a more cost-effective production strategy and help prioritize further development efforts. Thus, a general evaluation of the costeffectiveness of modular biofuel production is important considering all requirements and criteria.

\section{Methodology}

The research method was designed to support the goal of enabling further development of an approach for the manufacturing, placement, and optimal sizing of FPUs for biofuel energy production. The focus of this research is to rank stakeholders' requirements based on SME 
evaluation, to analyze FPUs alternatives to determine how they meet those requirements, and to determine how sensitive the result is to changes in stakeholders' priorities.

The research begins by inquiring about the identified requirements from the previous work about the biofuel manufacturing industry, which on the perspectives-level analysis. The model was developed using Logical Decision software V7.2. This software is adopted due to its advanced sensitivity analysis capability. In this model, BPSs' requirements directly connected to the overall goal.

Ten SMEs were interviewed individually to evaluate the eighteen requirements as well as the three FPUs' sizes. These ten SMEs were recommended based on consultation with the management team from the Iowa State University Bioeconomy Institute. The SMEs group for this research included academics researchers and industry representatives all with a minimum of 8 years of experience in the field.

The SMEs evaluated the importance of each of the eighteen requirements using a threelevel scale (high importance, medium importance, low importance). The interviewer explained to the SMEs the three levels of evaluation as follows:

- High importance: A small change in this requirement will have a significant measurable impact on the recommended biofuel production strategy.

- Medium importance: A change in this requirement will have a measurable impact on the recommended biofuel production strategy.

- Low importance: A change in this requirement may influence the recommended biofuel production strategy.

Then, the average evaluation is calculated using Equation 5.1. 
Where:

$$
\text { Requirement's weight }=\frac{3 *(\# \text { of } S M E S 1)+2 *(\# \text { of } S M E S 2)+1 *(\# \text { of } S M E S 3)}{\text { Total number of } S M E s}
$$

SMEs 1: is the group of those who weighted the requirement's importance as high.

SMEs 2: is the group of those who weighted the requirement's importance as medium.

SMEs 3: is the group of those who weighted the requirement's importance as low.

The ten SMEs evaluated each of the FPUs against each individual requirement using the 2,000 tpd FPU as the base-case since this is the most studied unit size as mentioned in the literature. The SMEs looked at the $200-500$ tpd FPU and was asked if it would have an advantage, be the same, or be at a disadvantage in meeting this requirement when compared to the 2,000 tpd FPU base case. A five level scale was used (big advantage - advantage - same disadvantage - big disadvantage). During the data collection, each SME were interviewed individually. In addition, SMEs were able to provide comments and ask the interviewer for some clarification when needed. This process was then completed with the FPUs that consume 50 tpd of feedstock.

To determine which FPU provided the best fit for stakeholders' requirements, the raw data collected from the SMEs on evaluation of the requirements using the five level scale was converted to numerical scales as shown in Table 5.2. This is done to transfer the qualitative evaluation to a quantitative one to have a numeric score representation for each requirement evaluation.

Table 5.1. Code for Requirements' Evaluation Assessment Scores

\begin{tabular}{|c|c|c|c|c|c|}
\hline The code: & Big advantage & Advantage & Same & Disadvantage & Big disadvantage \\
\hline & 2 & 1 & 0 & -1 & -2 \\
\hline
\end{tabular}


The raw data on the performance of each FPU against the base case was then calculated by getting the average value of the ten SMEs evaluations for each requirement as shown in Equation 5.2.

$$
\text { Avg. of requirement's scores }=\frac{\sum_{i=1}^{10} S M E_{i}}{\text { Total number of } S M E S}
$$

After that, each requirement's score at each FPU is calculated by using Equation 5.3.

Requirement's score $=$ Avg. of requirement's scores $*$ Requirement's weight

Next, the score of each FPU is calculated by using Equation 5.4.

$$
\text { FPU's score }=\sum_{i=1}^{18}\left(\text { Requirement }_{i} \text { score }\right)
$$

Then, these scores normalized using Equation 5.5. After that, these normalized data were entered into the developed model using direct entry function in the Logical Decisions V7.2 software.

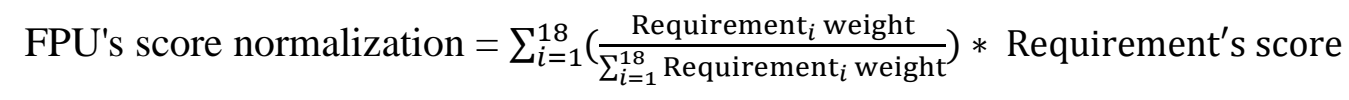

The model was then run and utilities were calculated. To calculate the utility of each alternative, Logical Decisions software uses functions called Single-measure Utility Functions (SUFs) which convert measure levels to utilities as explained in the software help. For the three FPUs alternatives, the one with the highest utility would indicate a better fit to stakeholders' requirements and the one with the lowest utility would indicate the poorest fit.

Then, the requirements evaluation by SMEs is used to prioritize the set of identified requirements from high importance levels to the lower importance levels. It is assumed that any 
requirement had a score of at least 2.5 out of 3 is considered as a high-priority requirement. This means at least 6 out of the ten SMEs evaluated this requirement as a high-priority requirement.

Finally, the sensitivity analysis was conducted on the model using the Logical Decisions software V7.2 (Biggam, 2011). This type of analysis explores the response of the overall utility of alternatives to changes in the relative importance (weight) of each requirement (Biggam, 2011). Logical Decisions software helps to make decisions based on MCDA. In addition to its uses in prior work in the analysis of biofuel stakeholders' perspectives for FPUs alternatives (Fawzy \& Componation, 2015), it has been used in fields such as health and environmental management (Cipollini, Maruyama, \& Zimmerman, 2005; Moffett, Dyer, \& Sarkar, 2006, Honoré, Fos, Smith, Riley, \& Kramarz, 2010). This software allows evaluating alternative solutions by considering multibal requirments simultaneously, which simplifies the decisionmaking proces with logical illustration.

\section{Results}

The analysis investigated both the results of the MCDA model and the comments from the SMEs. The smallest FPU's size, with a capacity of $50 \mathrm{tpd}$, is the best option based on the study that identified the five perspectives. This research used the eighteen stakeholders' requirements identified in the previous research (Fawzy \& Componation, 2015) to create the model. As mentioned in the methodology, all requirements in the model are connected directly to the overall goal, without the clustering on the sub-objectives. Then, all the weights were assigned by using the direct entry function in the software. Moreover, Logical Decisions V7.2 is used in this analysis. Figure 5.2 presents the final hierarchy with assigned weights. 


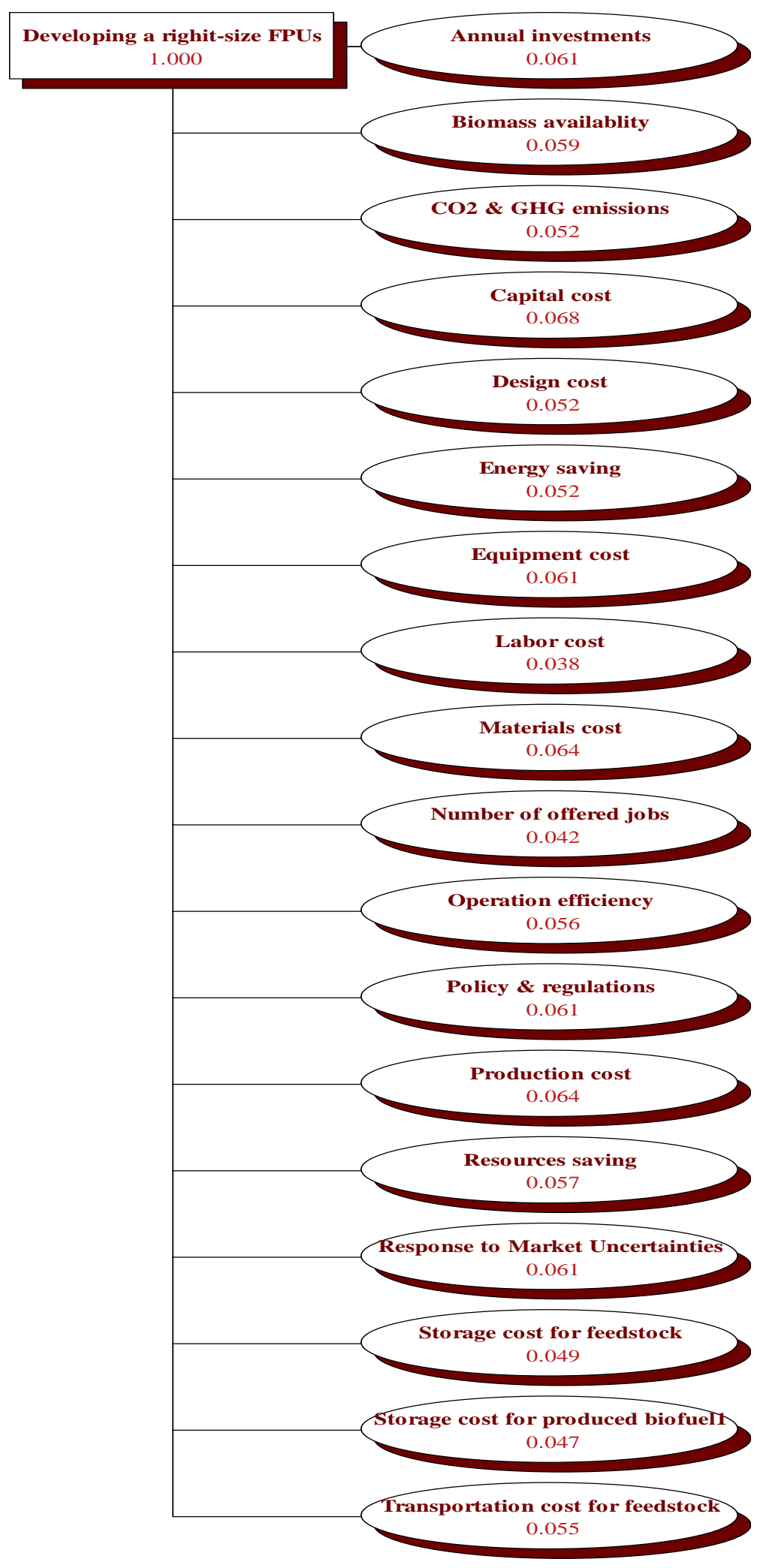

Figure 5.1. Final hierarchy with assigned weights using Logical Decisions ${ }^{\circledR}$ V7.2 
After analyzing the model shown in Figure 5.2, FPU with a capacity of 50 tpd was found as the best selection with a utility equal to 0.523 . This result coincided with the result of the previous study which conducted on the perspectives-level (Fawzy \& Componation, 2015). Similarly, FPU with a capacity between 200 - 500 tpd became the second best option with a utility equal to 0.519 . Finally, FPU with a capacity of at least 2000 tpd was found as the least selection with a utility equal to 0.500 . Figure 5.3 presents the three alternatives ranking and utilities for the model based on the stakeholders' requirements analysis.

Alternative Utility

$1.50 \mathrm{t} / \mathrm{d} \quad 0.523$

2. $500-200 \mathrm{t} / \mathrm{d} \quad 0.519$

3. $2000 \mathrm{t} / \mathrm{d} \quad 0.500$

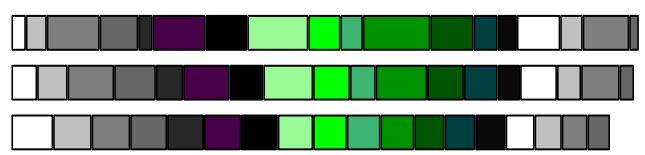

Capital cost Annual investments

Policy \& regulations Operation efficiency

$\square \mathrm{CO}_{2}$ \& $\mathrm{GHG}$ emissions

Storage cost for produced biofuel

\section{Production cost}

Equipment cost

Biomass availablity

Transportation cost for feedstock

Energy saving

$\square$ Number of offered jobs
Materials cost

Response to market uncertainties

Resources saving

Design cost

Storage cost for feedstock

Labor cost

Figure 5.2. Final utilities for the alternatives analysis from the Logical Decisions ${ }^{\circledR}$ model

It is noteworthy to mention that the equipment cost requirement is considered as the cost of the first group of equipment to run the facility plus any replacement or upgrades during the facility lifetime. The requirement of response to market and policy uncertainties means that the unit's flexibility and its ability to respond to demand and other market and political changes. Furthermore, the annual investment requirement represents the maintenance cost and the investors' ability to invest in such process annually. As stated by a SME, maintenance cost on average equals to $5 \%$ of overall cost, even the SMEs evaluated this requirement as a highprioritized one. Incidentally, according to some SMEs, transportation cost represent 
approximately $10 \%$ of the total cost, storages cost for raw material is around $22 \%$ and for final product it is represents another $10 \%$ to $17 \%$ of the total cost.

Two of the SMEs from the industry sector believe that the medium size facility is the best alternative and the smallest FPUs will lose the advantages of scale. Moreover, one of those two SMEs stated that this is not a linear decision. Instead of considering which size fit the stakeholders' requirements best, stakeholders should decide to start manufacturing the smallest size then duplicate it or produce bigger ones as needed. In other words, the first unit serves as an experimental unit to examine the situation and improve the learning curve.

For this research, the model is created using the Logical Decisions software V7.2 by considering all the eighteen requirements and their weights evaluated by the ten SMEs. The weights given to the requirements in the software using the direct enter method. As a result the small FPU has the highest utility comparing to the other alternatives. Thus, it is selected as the best alternative according to the stakeholders' requirements and the requirements evaluation of the three alternatives.

Figure 5.4 shows the results of more investigations on the requirements and their priorities. The eighteen requirements ranking from the highest importance to the lowest one are shown. According to the SMEs' evaluations, eight requirements had a score of at least 2.5 out of 3, which is considered a high-priority requirement. Thus, eight out of eighteen requirements get high-prioritized importance based on the ten SMEs evaluation. As shown in Figure 5.4, the capital cost requirement is evaluated as the highest important requirement for the decisionmaking with score of 2.9 out of 3 . This means that this requirement has the highest influence on the decision were nine out of the ten SMEs ranked it with high importance evaluation. Following the capital cost, both requirements of materials cost (feedstock cost) and production 
cost got the score equal to 2.7 out of 3 . Then, four of the eighteens evaluated requirements got a score equal to 2.6 out of 3 . These four requirements are: Policy \& regulations requirement, annual investments (maintenance) requirement, the unit's response to market \& policy uncertainties requirement, and equipment cost requirement. Finally, the biomass availability requirement, which is based on the current situation, is considered as a high-priority requirement with score equal to 2.5 out of 3 , which represent the evaluation of 6 out of 10 SMEs. These high-priority requirements are the ones that are examined in the sensitivity analysis.

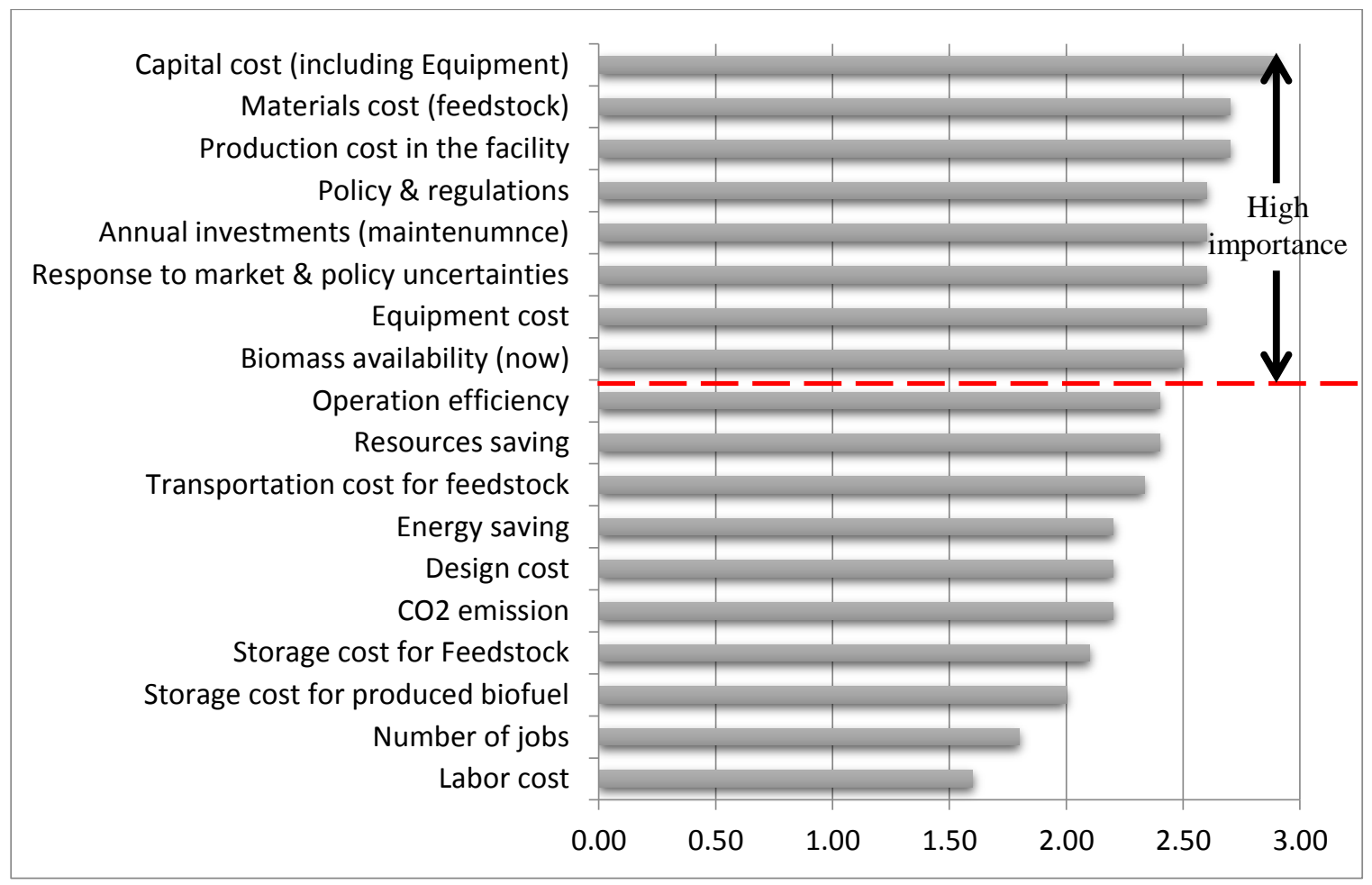

Figure 5.3. Ranked requirements of FPUs' manufacturing

\section{Sensitivity Analysis}

To study the result robustness, sensitivity analysis is conducted. Sensitivity analysis on the requirements level helps to understand which requirement(s) affecting the decision exactly 
under the general perspectives. In addition, to know under which condition(s) the decision will change. Moreover, what will be the best alternative for other situations, if exist.

A sensitivity analysis is applied on the requirements using Logical Decisions V7.2. As an output from this research, eight out of the eighteen requirements were ranked as highprioritized requirements according to the SMEs evaluation. Since the sensitivity analysis in this research is focused more on the high-prioritized requirements, it was done on these eight highprioritized requirements. When a sensitivity analysis was performed on each requirement of these eight, the weight given to that requirement is adjusted up or down to examine when the utilities ranking will change. On the other hand, the weights of to the other seventeen requirements in the model are adjusted proportionally. Since the capital cost has the highest score in the requirements importance evaluation by SMEs, a sensitivity analysis was conducted on this requirement first (Figure 5.5). The weight of the capital cost requirement was varied from 0 to $100 \%$. The sensitivity analysis showed that when the capital cost requirement was weighted at any value from 0 to $11 \%$ the smallest FPUs $(50 \mathrm{tpd})$ was recommended. When the weight given to the capital cost requirement was increased to $12 \%$, the medium sized FPUs (200 to 500 tpd) became the recommended size. When weight of the capital cost requirement exceeds $16 \%$, the big sized FPUs (at least 2000 tpd) were recommended. The vertical line at weight of $7 \%$ was the weight given to the capital cost requirement in the initial analysis and at this point, the smallest FPUs is the recommended unit.

Nine out of the ten SMEs believe that this requirement is the most important one for any kind of manufacturing. Moreover, some SMEs mentioned that this is the reason behind the focus of previous research on this requirement more than other ones. 


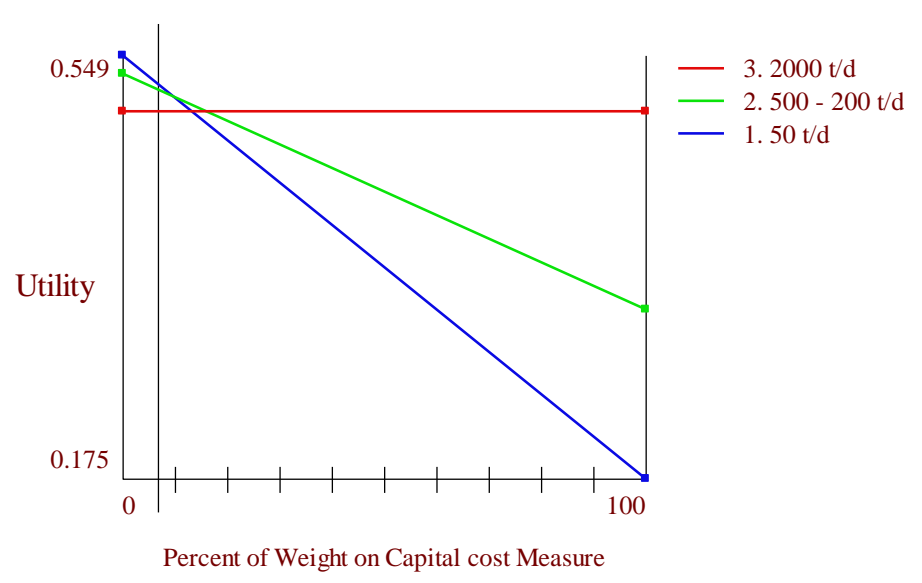

Figure 5.4. Sensitivity analysis on capital cost requirement

The same analysis was conducted on each one of the other high-prioritized requirements on sequence (Figure 5.6). This is done to examine the decision robustness. It is interesting to note that the smallest FPU is recommended in approximately $43(53.75 \%)$ of the 80 sensitivity analysis cases. When policy and regulations, raw material cost, or flexibility requirements were weighted no more than $4 \%$, the small FPU was recommended. An increase in the weights of the other requirements such as: production cost or equipment cost requirements resulted in the big FPUs becoming the recommended alternative when the weight increases by approximately $20 \%$ or more. For the biomass availability requirement, based on the current situation, as shown in Figure 5.6, the 50-tpd units is the best alternative when the importance of this requirement becomes more than 5\%. This result matches the first impression of most of the SMEs, where they believe that the smallest FPUs will have the advantages to be located wherever the feedstock is available. This is one of the strongest advantages of having such a distributed system. A summary of all requirements' weights and their impacts on the recommended alternative are shown in Figure 5.6. 


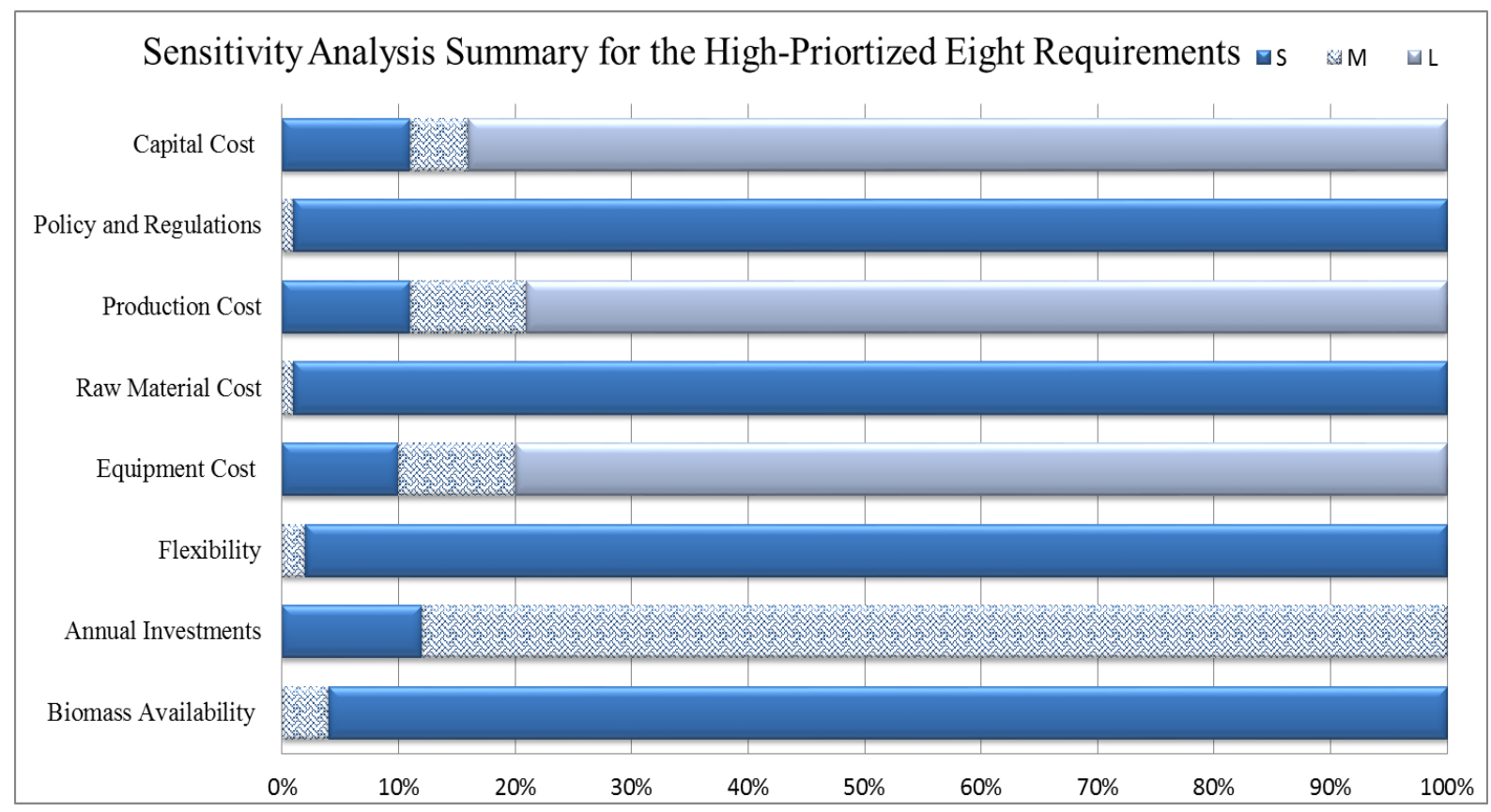

Figure 5.5. Sensitivity analysis summary for the high-prioritized eight requirements

It is also worthy to note that some SMEs mentioned that the production cost in the facility is also one of the most important requirements affecting decision after the capital cost requirement. Seven out of the ten SMEs believe in that the raw materials cost (feedstock cost) requirement is one of the factors that has a significant impact on the decision-making process, because there is a strong positive relation between this type of cost and the final product price. On the other hand, other SMEs believe that this requirement is not that important due to the units' flexibility advantage in addition to the availability of this raw material for biofuel production. This requirement shows the impact of the input side of the operation on the decision-making process. However, if this requirement has no weight value, the medium and smallest FPUs alternatives will have the same utilities in the sensitivity analysis. 
Due to the convergence of utilities between the smallest units' size alternative and the medium units' size alternative, the relationship between these two alternatives was studied further. Figure 5.7 shows that overall the smaller unit's size of FPUs (50 tpd) has a slightly better advantage than the medium FPUs size $(500-200$ tpd $)$. More specifically, it shows that the medium size of FPUs, due to its distribution, is worst in the transportation cost of raw material, biomass availability, and number of jobs offered by the facility. Moreover, the advantages of requirements such as material cost, raw material storages cost, response to market and policy changes is minimal. Figure 5.7 illustrates all the advantages and disadvantages on the requirements level base for both small and medium FPUs' sizes using tornado chart.

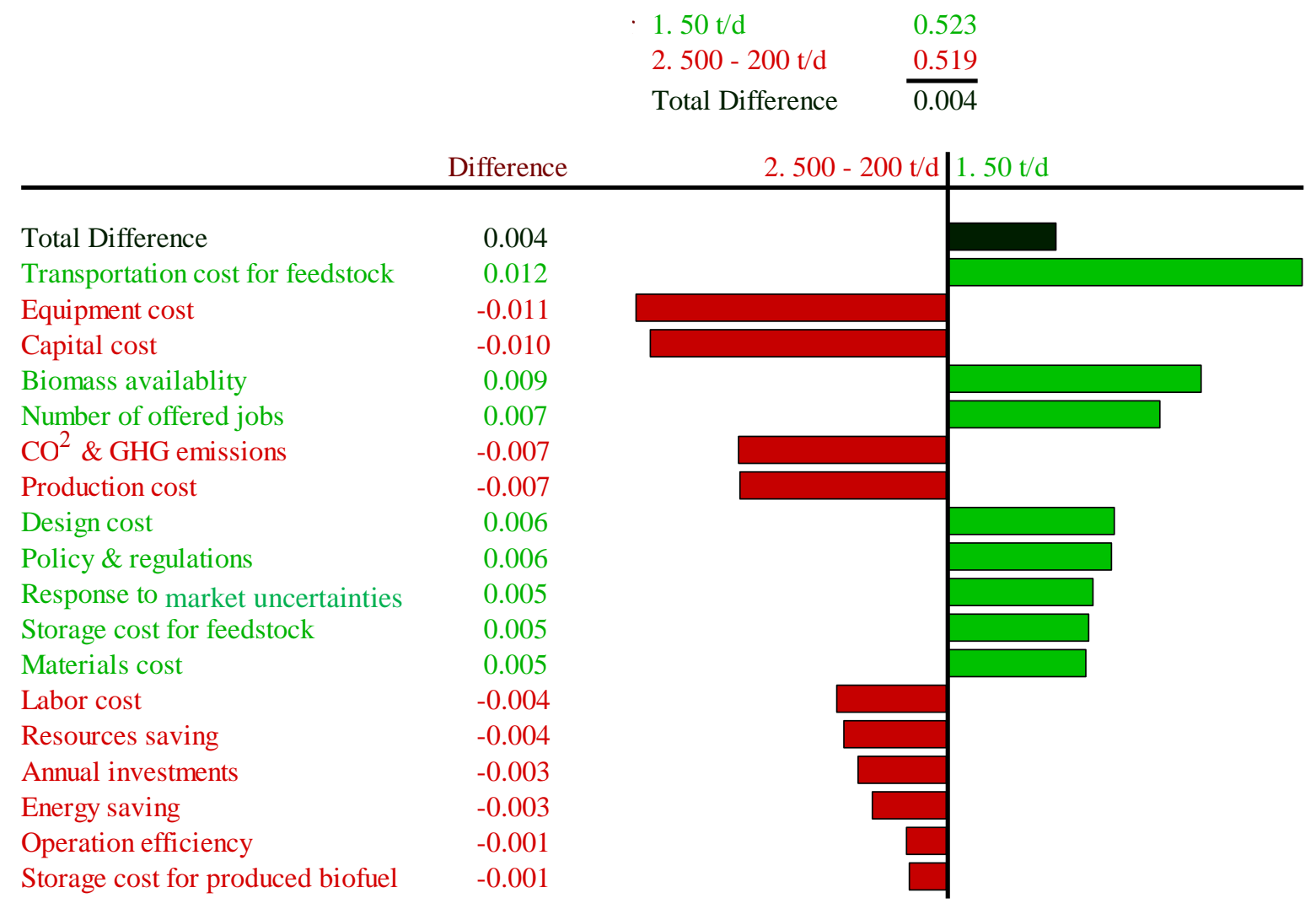

Figure 5.6. Sensitivity analysis on best two FPUs' sizes 
An industrial SME mentioned that some of the requirements could depend on others. He argued that equipment costs could be part of the capital cost and labor cost could be part of the production cost. As a part of the sensitivity analysis, the authors decided to remove these two requirements which could be related to capital and operation costs and rerun the model to see the impact of this action. As shown in Table 5.3, the smallest units' size got greater value (0.2313 out of 1$)$ than what it had with the existence of the two removed requirements (0.0934). In this case, removing these requirements did not affect the final decision. In fact, it did support the decision.

Table 5.2. Scores for the Alternatives Analysis under SME's Assumptions

\begin{tabular}{|c|c|c|c|c|c|c|c|c|c|c|c|c|c|c|c|}
\hline \multirow[t]{2}{*}{ Requirements } & \multicolumn{4}{|c|}{ Priorities } & \multirow{2}{*}{ 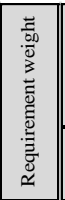 } & \multicolumn{3}{|c|}{$\begin{array}{c}\text { Alternatives (Facility size) } \\
\text { evaluations average } \\
\text { Pugh Concept - feedstock inputs tpd }\end{array}$} & \multicolumn{3}{|c|}{$\begin{array}{l}\text { Alternatives (Facility size) } \\
\text { The weighted Scores } \\
\text { Pugh Concept - feedstock inputs tpd }\end{array}$} & \multirow{2}{*}{ 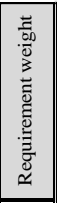 } & \multicolumn{3}{|c|}{$\begin{array}{c}\text { Alternatives (Facility size) } \\
\text { The weighted Scores } \\
\text { Pugh Concept - feedstock inputs tpd }\end{array}$} \\
\hline & $\mathrm{H}$ & $\mathrm{M}$ & $\mathrm{L}$ & \begin{tabular}{|c|} 
no \\
answer
\end{tabular} & & \begin{tabular}{|c|}
$\geq 2000$ tpd \\
(base)
\end{tabular} & $\begin{array}{c}200-500 \\
\text { tpd }\end{array}$ & $\leq 50$ tpd & \begin{tabular}{|c|}
$\geq 2000 \mathrm{tpd}$ \\
(base)
\end{tabular} & $\begin{array}{c}200-500 \\
\text { tpd }\end{array}$ & $\leq 50$ tpd & & $\mid$\begin{tabular}{|c|}
$\geq 2000$ tpd \\
(base)
\end{tabular} & $\begin{array}{c}200-500 \\
\text { tpd }\end{array}$ & $\leq 50$ tpd \\
\hline $\mathrm{CO}_{2} \mathrm{em}_{2}$;sion & 4 & 4 & 2 & 0 & 2.20 & 0 & 0.1111 & -0.4444 & 0 & 0.2444 & -0.9778 & 0.06 & 0 & 0.0064 & -0.0256 \\
\hline Resources saving & 5 & 4 & 1 & 0 & 2.40 & 0 & 0.1250 & -0.1250 & 0 & 0.3000 & -0.3000 & 0.06 & 0 & 0.0078 & -0.0078 \\
\hline Design cost & 4 & 4 & 2 & 0 & 2.20 & 0 & 0.3333 & 0.7778 & 0 & 0.7333 & 1.7111 & 0.06 & 0 & 0.0192 & 0.0448 \\
\hline Capital cost (including Equipment) & 9 & 1 & 0 & 0 & 2.90 & 0 & -0.7000 & -1.3000 & 0 & -2.0300 & -3.7700 & 0.08 & 0 & -0.0531 & -0.0986 \\
\hline Production cost in the facility & 7 & 3 & 0 & 0 & 2.70 & 0 & -0.4444 & -0.8889 & 0 & -1.2000 & -2.4000 & 0.07 & 0 & -0.0314 & -0.0628 \\
\hline Materials cost (feedstock) & 7 & 3 & 0 & 0 & 2.70 & 0 & 0.4000 & 0.7000 & 0 & 1.0800 & 1.8900 & 0.07 & 0 & 0.0282 & 0.0494 \\
\hline Transportation cost for feedstock & 5 & 2 & 2 & 1 & 2.33 & 0 & 1.1111 & 2.0000 & 0 & 2.5926 & 4.6667 & 0.06 & 0 & 0.0678 & 0.1221 \\
\hline Response to market \& policy uncertainties & 7 & 2 & 1 & 0 & 2.60 & 0 & 0.5556 & 0.8889 & 0 & 1.4444 & 2.3111 & 0.07 & 0 & 0.0378 & 0.0604 \\
\hline Storage cost for feedstock & 3 & 5 & 2 & 0 & 2.10 & 0 & 0.5000 & 0.9000 & 0 & 1.0500 & 1.8900 & 0.05 & 0 & 0.0275 & 0.0494 \\
\hline Storage cost for produced biofuel & 4 & 1 & 4 & 1 & 2.00 & 0 & -0.2222 & -0.3333 & 0 & -0.4444 & -0.6667 & 0.05 & 0 & -0.0116 & -0.0174 \\
\hline Annual investments (maintenumnce) & 6 & 4 & 0 & 0 & 2.60 & 0 & 0.3000 & 0.1000 & 0 & 0.7800 & 0.2600 & 0.07 & 0 & 0.0204 & 0.0068 \\
\hline Energy saving & 3 & 6 & 1 & 0 & 2.20 & 0 & -0.5000 & -0.7000 & 0 & -1.1000 & -1.5400 & 0.06 & 0 & -0.0288 & -0.0403 \\
\hline Biomass availability (now) & 6 & 3 & 1 & 0 & 2.50 & 0 & 0.8000 & 1.4000 & 0 & 2.0000 & 3.5000 & 0.07 & 0 & 0.0523 & 0.0915 \\
\hline Operation efficiency & 4 & 6 & 0 & 0 & 2.40 & 0 & -0.5000 & -0.6000 & 0 & -1.2000 & -1.4400 & 0.06 & 0 & -0.0314 & -0.0377 \\
\hline Policy \& regulations & \begin{tabular}{|l|l|l}
6 & \\
\end{tabular} & 4 & 0 & 0 & 2.60 & 0 & -0.1250 & 0.2500 & 0 & -0.3250 & 0.6500 & 0.07 & 0 & -0.0085 & 0.0170 \\
\hline \multirow[t]{2}{*}{ numNumber of jobs } & 2 & 4 & 4 & 0 & 1.80 & 0 & 1.0000 & 1.7000 & 0 & 1.8000 & 3.0600 & 0.05 & 0 & 0.0471 & 0.0800 \\
\hline & & & & & & & \multicolumn{2}{|c|}{ FINAL SCORS: } & 0 & 5.7254 & 88444 & 1 & 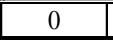 & 0.1497 & 02313 \\
\hline
\end{tabular}


For this research, three types of sensitivity analysis are conducted. First sensitivity analysis is done to examine the result robustness. This sensitivity analysis is done by changing each requirement weight from $0 \%$ (no importance give to the requirement) to $100 \%$ (all the importance give to the requirement). Then, authors observe the effect of these changes on the result. From this analysis authors found that small FPU size has the advantage in four out of the eight high-prioritized requirements. Second sensitivity analysis is done to examine differences between the best two alternatives, which are the small and medium FPUs sizes. This type of sensitivity analysis done using tornado chart to illustrate the advantages and disadvantages of each of the two FPUs sizes comparing to each other based on the requirements-level. As a result for this analysis, the small FPU size got the advantage at eight out of the eighteen requirements comparing to the medium FPU size. Last sensitivity analysis is done to investigate the dependency of two of the requirements according to an industrial SME argument. This type of sensitivity analysis is done by using a Microsoft Excel model by excluding the two requirements. As a result of this sensitivity analysis, authors found that the smallest FPU size got the highest score again comparing to the other two alternatives.

\section{Conclusion and Recommendations}

This research supports the decision making process by providing insight on how stakeholder requirements influence selection of the appropriate facility size for biofuel production. From requirements-level point of view, the smallest FPUs' size is the best option for the eighteen requirements, but under specific conditions. From the investigation on the requirements-level the authors found that eight out of the identified eighteen requirements as were ranked as high-prioritized requirements according to the SMEs evaluation. those eight high-prioritized requirements consecutively are: (1) Capital cost, (2) raw materials cost (the 
feedstock), (3) production cost in the facility, (4) policy and regulations, (5) annual investments, (6) response to market and policy uncertainties and changes, (7) equipment cost, and (8) biomass availability (in the current situation), as shown in Figure 5.4.

Three out of the eight highly important requirements present the big FPUs alternative as the best alternative under a wide range of weights. In other words, for capital, production, and equipment costs, the big FPUs alternative is recommended whenever the weight of each of these requirements exceed approximately one fifth of the total requirements weights. From this research, it is recommended that more work is needed to focus on reducing capital, production, and equipment costs for FPUs manufacturing. This will avoid the economics of scale concept and reduce these costs variation among the three FPUs alternatives sizes. Using appropriate application of advanced manufacturing technologies is one possible approach to use, since the study of advanced manufacturing technologies and its relationship with business' strategy is currently receiving significant scholarly attention (Kotha \& Swamidass, 2000). In addition to that thinking small in design and manufacturing perceptions is another approach that could be followed (Dahlgren, Göçmen, Lackner, \& van Ryzin, 2013). The combination of these two approaches could also be effective in reducing capital, production, and equipment costs.

Four out of the eight high-prioritized requirements presented the small FPUs size as the best option whenever each requirement weight exceeded the 5\%. Overall, the medium FPUs in the analysis became the second best alternative with a close utility to the first alternative. However, only one requirement of the eight high-prioritized requirements presented the medium unit's size as the best option for FPU manufacturing with a wide range of weights. This requirement is the annual investment requirement including the maintenance cost of the facility. This helps to think about the available advantages of maintenance cost for the medium 
unit's size and how it could be implemented in the smallest unit's size by encourage engineers to think small in design and manufacturing perceptions as Dahlgren and his colleague (2013) recommended.

Authors believe this research support the idea that "bigger is not always better." This research highlights some requirements' impacts on the decision. Moreover, it paved a way to select the unit's size for biofuel production based on a scientific methodology for the decisionmaking considering stakeholders' requirements.

Even though the smallest FPUs size is the best units' size to pass both analysis levels of perspectives, from Fawzy and Componation's study (2015), and requirements, from the current research, the biggest FPUs size is recommended as the second best alternative in $37.5 \%$ (3 out of 8 requirements) of the high-prioritized requirements, from the current research. On the other hand, the medium FPUs size is recommended as the second best alternative based on the perspectives'-level analysis as shown in Fawzy and Componation (2015). This deep analysis of the requirements-level provides a clear picture on what engineers should focus on if they want to think small in design and manufacturing perceptions. Moreover, this work illustrates the effect of each of the top eight requirements on the FPUs production decision and which of them could be improved to reach smaller verses bigger FPUs. 


\title{
CHAPTER 6. A Multi-Criteria Decision Framework for an Unstructured Complex Problem: Biofuel Unit Manufacturing
}

\author{
A paper submitted to Applied Energy Journal
}

Mostafa F. Fawzy, Caroline C. Krejci, Paul J. Componation, and Guiping Hu

\begin{abstract}
This paper focuses on an unstructured complex problem assessment. A comprehensive analysis was carried out on six decision-making (DM) tools: Pugh Concept Selection Matrix, Weighting Sum Method (WSM), Simple Multi-Attribute Rating Technique (SMART), Technique for Order of Preference by Similarity to Ideal Solution (TOPSIS), Analytic Hierarchy Process (AHP), and ELimination and Choice Expressing REality (ELECTRE). Their advantages and disadvantages, in addition to their applications in the renewable energy and biofuel manufacturing industry, were demonstrated. A framework of multi-criteria decision analysis (MCDA) was utilized to select the best Fast Pyrolysis Units (FPU) that fits the stakeholders' perceptions. This decision was based on assessing and measuring three different sizes of FPU for biofuel production using Pugh Concept Selection Matrix. The FPU selection process was conducted using integrated versions of MCDA based on different DM tools. This research proposed the integration between Pugh Concept Selection Matrix and WSM as one integrated approach. Moreover, it proposed the integration between Pugh Concept Selection Matrix and TOPSIS as another integrated approach. Finally, this research compared results from these two
\end{abstract}


integrated approaches and the result from a previous proposed integrated approach which integrated Pugh Concept Selection Matrix, WSM, and sensitivity analysis using Logical Decision® software to solve the same biofuel production problem. As a result, all three approaches recommended the small FPU as the best alternative that meets stakeholders' needs. However, each approach provided a different insight about the problem and the available alternatives, which helps the decision makers discuss these alternatives with clear vision.

Keywords: Multi-Criteria Decision-Analysis, Decision-Making, Biofuel, Pyrolysis.

\section{Introduction}

Biofuel is a renewable alternative for traditional energy production. One method of biofuel production is fast pyrolysis, which is a thermochemical process in which biomass, or any other carbonaceous material, is converted into char, bio-oil and non-condensable gases (Fawzy \& Componation, 2015b). The manufacturing of Fast Pyrolysis Units (FPUs), which is a facility used to produce biofuel via fast pyrolysis process, has been studied previously under limited scopes by focusing on the analysis of logistic costs to determine optimal size of a biofuel refinery (Larasati, Liu, \& Epplin, 2012) and further work is needed to continue biofuel development. One particular challenge for biofuel development is the number of diverse biofuel production stakeholders (BPS) who are involved in the decision of manufacturing FPUs (Fawzy \& Componation, 2014). Each BPS group has different perspective(s) and requirements (Fawzy \& Componation, 2015a).

Determining the right size of an FPU is defined as a complex problem (Fawzy \& Componation, 2015a). This right size is a FPU that should meets all or most of the stakeholders' perception. Complex problems are typically evaluated using mathematical modeling methods, such as optimization and simulation, or multi-criteria decision analysis (MCDA) methods. 
However, using mathematical models to assess FPU sizes is often impractical, due to the lack of complete information in this conceptual stage. On the other hand, MCDA methods have become progressively popular in renewable energy decisions to accommodate the different stakeholder perspectives and the inherent system complexity (Pohekar \& Ramachandran, 2004; Wang et al., 2009). In general, problems characterized by limited information, multiple stakeholders, and conflicting requirements are often solved by MCDA methods, such as multi-attribute utility theory (MAUT) (Fawzy \& Componation, 2015a).

In this study, multiple decision-making (DM) tools were identified through a review of research publications, and their application to decision problems in energy and the biofuel production industry specifically are identified. Next, comparisons among these DM approaches are conducted to highlight the pros and cons of each approach. After that, three of the DM tools were chosen to assess the biofuel production system and the FPU size selection problem based on previously-determined selection criteria. The problem identification stage, including an assessment of BPS perspectives and requirements regarding FPU size (i.e., the decision criteria) and the set of feasible unit sizes (i.e., the decision alternatives) was accomplished in our previous research (Fawzy \& Componation, 2015a). This analysis provides insights into the same problem from different points of view. Although we apply these DM processes specifically to biofuel production, they are also applicable to a range of similar open-ended problems that are common in engineering design. 
Literature Review

\section{Complex and Unstructured Decision Problems}

Complex decision problems cannot be solved using a simple logical process. This class of problems is defined as the case where: (1) The decision maker seeks to achieve multiple, often conflicting objectives, (2) the decision maker is faced with many alternative solutions to his decision problem, each of which has multiple attributes, (3) the problem is characterized by uncertainty, (4) the decision maker does not have perfect information about every aspect of the problem, and (5) complexity increases when there are multiple stakeholders involved (Grunig \& Kuhn, 2013). There are often disagreements among stakeholders about the relative importance of key objectives (Gregory \& Keeney, 1994).

Decision problems that can be described as "complex" are also often considered to be "unstructured." Simon and Newell (1958) introduced the concept of "well-structured" and "illstructured" problems. A well-structured problem can be formulated explicitly and quantitatively, with objectives that can be described in terms of a well-defined objective function (e.g., “maximize profit"), and can be solved algorithmically yielding a numerical solution. By contrast, an ill-structured problem contains objectives and decision variables that cannot be expressed numerically (i.e., they must be represented qualitatively), and solving such problems using standard algorithmic optimization techniques is infeasible (Simon \& Newell, 1958). Gorry and Scott Morton (1971) made a distinction between structured and unstructured decision problems and argued that the design of solution procedures will be very different for each type. They described unstructured problems as requiring human judgment, evaluation, and insights into problem definition (which is often ambiguous), whereas structured problems could be evaluated 
through the use of routine procedures and/or automation. In addressing unstructured decision problems, they also argued that providing the decision maker with more and/or better quality information would not necessarily improve the quality of their decisions - instead, they required decision-supporting methodologies (Gorry \& Scott Morton, 1971). Saaty (1978) differentiated structured and unstructured decision problems by focusing on the differences in how accurately risk and uncertainty could be quantified for the problem. Unstructured problems differ in that not only are the probabilities of each outcome unknown, but the nature of the outcomes themselves is unknown (Saaty, 1978). Researchers have argued that most important real-life decision problems are unstructured, particularly those faced by upper management in organizations, for whom strategic decision making is required (Simon \& Newell, 1958; Mintzberg, Raisinghani, \& Theoret, 1976; Saaty, 1978).

Many important complex decision problems that lack the structure necessary for analytical assessment can also be classified as "open-ended." An open-ended problem is one which could have multiple "optimal" answers, based on different stakeholders' perspectives and requirements, as well as a lack of complete and perfect information (Fawzy, Componation, \& $\mathrm{Hu}, 2015)$. This type of decision problem is one that has not been encountered previously and for which no predetermined and explicit set of solutions exists (Mintzberg, Raisinghani, \& Theoret, 1976).

\section{Complex and Unstructured Decision Problems in Renewable Energy}

In the domain of renewable energy management, there are many complex and unstructured decision problems that must be addressed. In their review of literature in the domain of energy modeling with environmental considerations, Huang et al. (1995) identified six 
categories of decision problems: Energy planning and policy analysis, site selection of power plants, technology choice and project appraisal, energy conservation, energy production and operation, and environmental control and management. These decision problems tended to focus on minimizing expected risk (in terms of cost, supply, or the environment) and/or maximizing energy supply security, economic benefits, or benefits to society. Most of the literature that they reviewed (31\%) fell into the category of energy planning and policy analysis, which involves many uncertain factors and long planning horizons (Huang, Poh, \& Ang, 1995). Zhou, Ang, and Poh (2006) categorized decision problems in renewable energy management more broadly as being either long-term strategic/policy decisions (e.g., policy analysis, investment planning, energy conservation strategy selection) or short-term operational/tactical decisions (e.g., bidding, pricing, technology choice). Most of the literature that they reviewed (63\%) fell into the category of strategic/policy decisions. In Pohekar and Ramachandran (2004), the following application areas for sustainable energy DM were identified:

- Renewable energy planning and energy resource allocation (e.g., investment planning, energy capacity expansion planning, evaluation of alternative energies)

- Building energy management (i.e., system design, selection, and installation, given multiple objectives)

- Transportation energy management (e.g., pollution control strategies, vehicle selection)

- Planning for energy projects (e.g., site selection, technology selection)

- Electric utility planning (e.g., electrical dispatch scheduling, power generation mix)

One factor that contributes to the complexity of these decision problems is the multiple and conflicting objectives that are involved. In particular, the traditional focus on minimizing costs must be augmented to account for environmental and social sustainability criteria (Wang et 
al., 2009). In complex projects like biofuel assessment, it is impossible to optimize all of these criteria at the same time (Perimenis et al., 2011). These decision problems are also characterized by high uncertainty, multiple stakeholders with varying perspectives, and data and information that exist in a variety of forms and degrees of completeness (Wang et al., 2009; Zhou, Ang, \& Poh, 2006). Additionally, decision makers must incorporate the complex interactions among economic, technological, ecological, and social systems that comprise the overall energy system (Wang et al., 2009). These decision problems are also often high-impact of irreversible with long time frames and capital-intensive investments (Huang, Poh, \& Ang, 1995).

Complex decision problems in the area of bioenergy have some aspects that are specific to the domain. In their review of the literature on MCDA methods for bioenergy systems, Scott et al. (2012) identify six categories of decision problems in the bioenergy domain: The technology to be used, fuel sources, methods for storing and transporting materials, the appropriate size and capacity of a project, facility location, and financial management of the project. According to Gnansounou (2011), biofuel decision problems are characterized by specific criteria, which he categorized into economic, environmental, and social areas of emphasis. Economic factors include the benefits attained at all stages of the biofuels value chain (including the feedstock producers) and the competitiveness and economic viability of biofuels (without government support). Environmental factors include appropriate land use to support biodiversity and protect natural ecosystems, natural resource consumption (especially water usage), greenhouse gas emissions, and impacts to water and air quality. Social factors include social control (i.e., the control that local communities retain over food security, land use rights, and inputs, especially seeds) and working conditions and worker safety. He described a wide range of stakeholders, including feedstock producers, biofuel producers, and the general public 
(Gnansounou, 2011). In assessing the specific problem of deciding on the best facility size for biofuels production, Fawzy and Componation (2015a) suggested that five categories should be considered: Economic, environmental, technical, legal, and social. These categories were based on the requirements that were identified by two specific sets of stakeholders: Academic researchers and biofuel industry representatives.

\section{MCDA Application in Renewable Energy}

Biofuel production as a complex problem needs a systematic approach to be solved. MCDA is particularly suitable for addressing complex energy management problems (Wang et al., 2009). MCDA is defined as "a collection of formal approaches which seek to take explicit account of multiple criteria in helping individuals or groups explore decisions that matter" (Belton \& Steward, 2002). MCDA methods can help improve the quality of decisions by making them more explicit, rational, and efficient. They can enable a better understanding of inherent features of the decision problem, promote the role of multiple participants in decision making processes, and facilitate compromise and collective decisions (Pohekar \& Ramachandran, 2004). However, selecting the most appropriate MCDA method to apply to a decision problem requires careful consideration, especially since different methods may provide different results. The decision maker(s) should choose a method that is easy to use and understand, that is compatible with the available data, that measures what the decision maker wants to measure, and that

provides the decision maker with all the information he / she needs to make a good decision (Loken, 2007).

Zhou et al. (2006) made a distinction between multi-objective decision making (MODM) models and multi-attribution decision making (MADM) models as sub-criteria under the general 
category of MCDA. The MODM models (i.e., decision problems with many or infinite alternatives) usually try to maximize/minimize an objective function, subject to constraints, and are typically solved using mathematical modeling/optimization methods (e.g., algorithms). Linear programming techniques, in combination with other methods for simultaneous consideration of multiple objectives, are the most frequently applied solution methodologies for these types of models (Scott, Ho, \& Dey, 2012). Although these models are useful in addressing some decision problems in renewable energy (e.g., determining an optimal blend of energy sources and fuel types, facility location), the MADM approach is required for addressing other decision problems in this domain, particularly those that are highly complex and ill-structured.

In contrast with the MODM models, in which an optimal or "best" solution is mathematically determined, MADM refers to models in which preference decisions are made by evaluating and prioritizing the alternatives, which are usually characterized by multiple conflicting attributes (Zhou, Ang, \& Poh, 2006). In this paper we will consider five of the mostcommonly used MADM methods: The Weighted Sum Method (WSM), the Simple MultiAttribute Rating Technique (SMART), the Analytic Hierarchy Process (AHP), the Technique for Order of Preference by Similarity to Ideal Solution (TOPSIS), and an outranking method known as the ELimination and Choice Expressing REality (ELECTRE). These techniques will be described and compared in detail in the Results section of this paper. Furthermore, the Pugh Concept Selection Matrix, which was used in prior work to collect data for the same problem (Fawzy \& Componation, 2015a), will be utilized in this study. 


\section{FPU Manufacturing Decisions}

FPUs were previously investigated and evaluated as an unstructured complex decision problem using an integrated MCDA approach. In this Prior work, 36 groups of stakeholders in the biofuel industry were identified. These stakeholder groups were clustered into five sets based on their background and interests (Fawzy \& Componation, 2014). In a related study, 18 requirements were identified as the ones that were most frequently mentioned in the literature (Fawzy \& Componation, 2015a). The sample for this study is assumed to be a group of SMEs in bioenergy where each of the five stakeholders' perspectives is represented by at least one SME of the sample. Both studies (Fawzy \& Componation, 2014; 2015a) were used for the problem identification and data collection for this work.

For biofuel facility manufacturing, previous research investigated three sizes of FPUs: Large, medium, and small. These three sizes were selected to be the alternatives for the study described in this paper. Each category is not actually a specific size but rather a range of biomass consumptions that are grouped in three sizes for investigation at this initial phase. Previous research assumed that the large facility size is a typical corn ethanol plant that consumes at least 2,000 tons per day (tpd) of biomass (Wright, Brown, \& Boateng, 2008). Many current research studies use 2000 tpd as their base case to allow for easy comparison with other work even though it may not be the optimal size for all technologies (Wright, Brown, \& Boateng, 2008; Anex, et al., 2010; Swanson et al., 2010; Wright et al., 2010). Therefore, in this study we assumed this size to be the base case for evaluation. The medium-sized unit was assumed to consume 200 to 500 tpd of feedstock, which has been proposed in previous work as an alternative that could be supported by a small group or cooperative of farmers (Ringer, Putsche, \& Scahill, 2006; Wright, Brown, \& Boateng, 2008; Wright et al., 2010). The capacity and costs for this size is comparable 
to the early ethanol and biodiesel plants. The small size unit was proposed to be the unit that consumes 50 tpd or less. Researchers advocate this as a viable alternative because it can be mobile, rather than built in a fixed location. FPUs of this size have been shown to be technologically feasible (LaClaire, Barrett, \& Hall, 2004).

As described by Wright and Brown (2007), there is an optimal plant size for the lowest unit cost of biobased product. This optimal size is still so large that capital investment for advanced biorefineries is estimated to be as much as a billion dollars. Prior work has also been done to develop frameworks to assess cost-effectiveness of alternative energy strategies (Qin et al., 2012). Using regular cost analysis techniques focuses only on the economic side of the problem. However, at least four other perspectives should be included in the biofuel manufacturing units decisions if not more (Fawzy \& Componation, 2015a).

\section{Methodology}

The goal of this research is to apply a set of DM tools to evaluate three different sizes of FPUs for biofuel production. Specific questions to be answered in this research effort (shown in Figure 6.1) include:

1. Which DM tools have been applied to complex problems in renewable energy, and more specifically, biofuel production?

2. What are the advantages and limitations of these DM tools?

3. Is there an individual or a set of DM tools that provide better insight into the complex problem of biofuel production?

4. Based on stakeholder perceptions, which FPU would be recommended for biofuel energy production? 


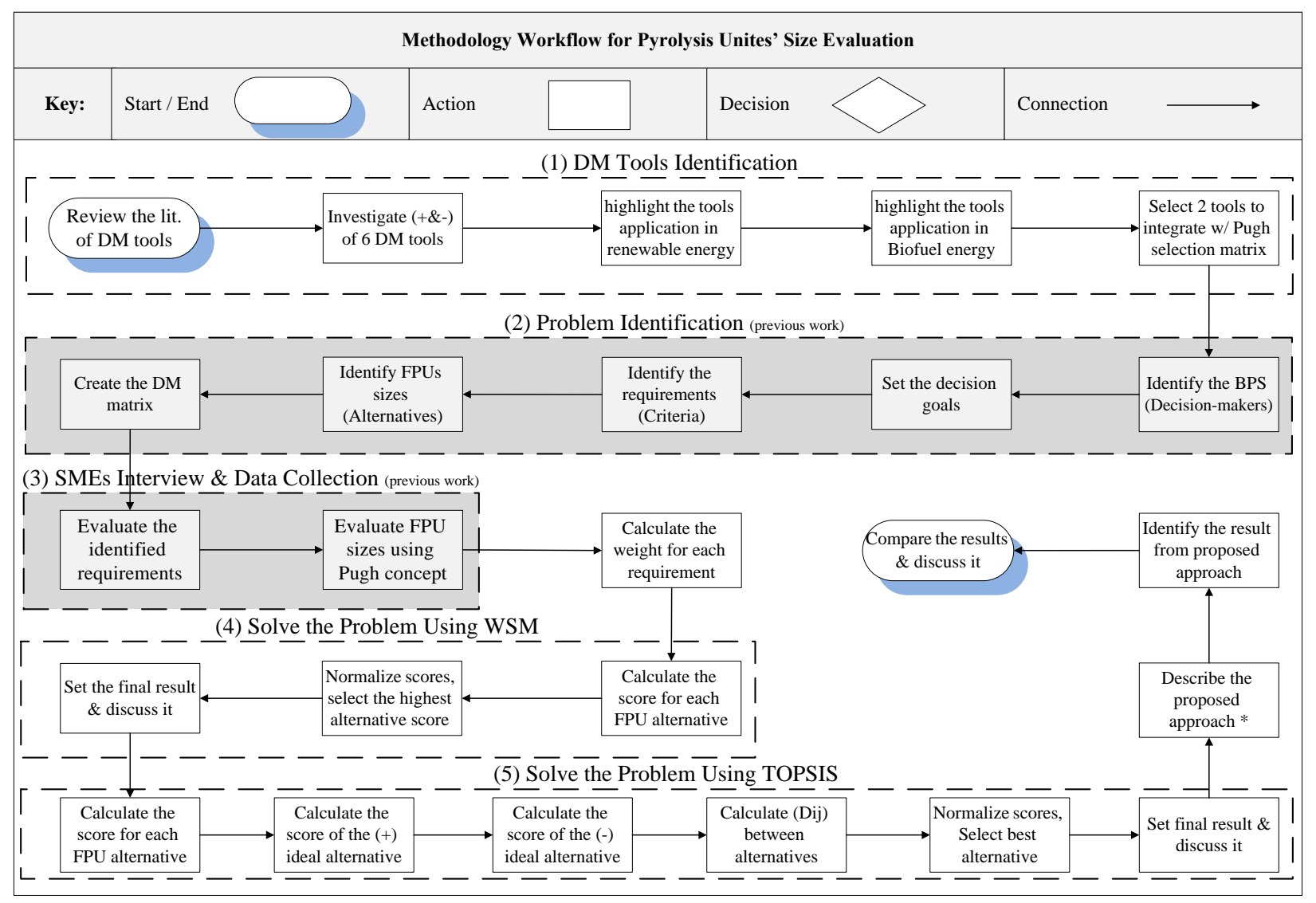

BPS $=$ Biofuel Production Stakeholders, DM= Decision-Making, FPU = Fast Pyrolysis Units, SME $=$ Subject Mater Experts, WSM= Weighting Sum Method, TOPSIS = Technique for Order of Preference by Similarity to Ideal Solution,

*Proposed approach= integration between: Pugh selection matrix, WSM, \& sensitivity analysis using Logical Decision software

Figure 6.1. Research methodology

The first task, "DM Tools Identification", is a review of peer-reviewed publications on the DM tools since 1986. The most commonly cited DM tools are selected for further analysis. Tool selection is based on frequency of use, ease of use, applicability to the problem, and the availability of needed data to use the tool. The advantages and disadvantages of each of the DM tools are discussed. For the purpose of the case study, we also looked at the DM tools' application to biofuel production. DM tools that are found to be the most applicable were selected for use of the biofuel production problem.

The second task is "Problem Identification." This task was completed in conjunction with prior research that identified the BPS (Fawzy \& Componation, 2014). It also included 
interviewing a group of BPS to help clarify the problem, review and provide feedback on possible DM criteria and viable biofuel production alternatives (Fawzy \& Componation, 2015a). The literature review also helped to develop a conceptual framework of the DM model including potential criteria and biofuel production alternatives: 2,000 tpd as the base case, 200 to 500 tpd as the medium sized unit, and 50 tpd as the small unit (Fawzy \& Componation, 2015a). Based on the interviews, a final DM matrix was developed. Inputs from the BPS were also used to identify Subject Matter Experts (SMEs) who would participate in the next phase of the research.

As part of the second task, a Pugh Concept Selection Matrix was selected to structure the problem for the SME interviews and data collection. The Pugh Matrix was used in this research because it can determine the right direction of the facility size for biofuel production without complex mathematical formulas (Cervone, 2009a). It is a paired comparison analysis (Cervone, 2009b) that can be used as a DM tool when there is little or no data available (Cervone, 2009a). It is a tool that works well when stakeholders are challenged with a problem that has multiple perspectives and requirements (Pugh, 1991). Another advantage of the Pugh Matrix for this application is that it is useful for decisions that deal with quantitative and qualitative data, in addition to uncertain environments and risky situations (Min, 1994; Cervone, 2009a).

The third task was the SME interviews and data collection, which had also been completed as part of a prior effort (Fawzy \& Componation, 2015a; Fawzy, Componation, \& Hu, 2015). Identification and selection of SMEs is not a trivial task. A study conducted to evaluate different alternatives for new energy system development used a team of 14 SMEs (Tzeng, Shiau, \& Lin, 1992). Another study evaluated the regional sustainability of bioenergy development had 13 bioenergy SMEs participate in the multi-stakeholder forum (Kurka, 2013). Therefore, we decided to invite 15 bioenergy SMEs for this study to evaluate the three FPUs 
alternatives. The SMEs were recommended based on consultation with the management team from the Iowa State University Bioeconomy Institute, and included academic researchers and industry representatives. The SME team members' experience ranged from 3 to 38 years, with an average of 13 years. SMEs also had advanced degrees in Food, Agricultural, Biorenewable Resources and Technology, Biological Engineering, Mechanical Engineering, Industrial Engineering, or Organic Chemistry. All SME team members have had prior experience in or are currently involved in biofuels research.

In general, data can be collected through observation or communication. Since this research assessed a conceptual model, and observational data is typically done on an existing process or experiment, it was not a viable option for this study. The communication approach is useful in examining attitudes, motivations, intentions, and expectations (Blumberg, Cooper, \& Schindler, 2008). The most common communication data collection methods are fact-to-face interviews, phone interviews, and self-administered inquiry. These methods can be used alternatively or in a combination (Blumberg, Cooper, \& Schindler, 2008; Biggam, 2008). Faceto-face interviews were conducted for this study because they allow the interviewer to gather indepth data and ask for further explanations as needed (Blumberg, Cooper, \& Schindler, 2008). In addition, it gives the SMEs the chance to ask for clarification of questions. A challenge is that face-to-face interviews require more labor in the data collection and when conducting follow-ups (Hussey \& Hussey, 1997; Blumberg, Cooper, \& Schindler, 2008). To avoid or at least minimize these disadvantages, researchers interviewed local SMEs. To allow elaboration, the interview had one open-ended question to allow the interviewee to discuss freely about perspectives and requirements that are important in biofuel production from their viewpoints. The interviewers were only able to successfully schedule 10 of the planned 15 interviews with SMEs. In each 
interview, the SME was asked to evaluate the priority of each requirement and then compare how each of the three FPU performs.

Detailed justifications of requirement weights are not always included in new energy evaluation studies. Part of that is because of the uncertainties associated with new energy developments, where performance of the alternatives is difficult to quantify (Tzeng, Shiau., \& Lin, 1992). In some cases, stakeholder inputs to evaluate the requirement weights are done as separate task prior to the actual energy policy study (Browne, O'Regan, \& Moles, 2010). Others used a pair-wise comparison technique to ask stakeholders to compare the importance of criteria (Wang et al., 2009). Another approach is the use of voting technique such as Noguchi's voting and ranking methods to determine the criteria orders instead of weights (Liu \& Hai, 2005).

In this study, SMEs were interviewed individually and their responses were recorded and later transcribed by the interviewer. The SMEs were asked to place each requirement in one of the three groups: High importance, medium importance, and low importance. The interviewer explained to the SMEs the three levels of evaluation as follows:

- High importance: A small change in this requirement would have a significant measurable impact on the recommended biofuel production strategy.

- Medium importance: A change in this requirement would have a measurable impact on the recommended biofuel production strategy.

- Low importance: A change in this requirement may influence the recommended biofuel production strategy.

To determine which FPU provided the best fit for stakeholders' perspectives and requirements, the raw data collected from SMEs on requirements ranking were converted to numerical scales. The weight of each requirement was performed using Equation 6.1. Then, the 
raw data on the performance of each FPU using Pugh Matrix were converted to a numerical score.

$$
\text { Requirement's weight }=\frac{3 *(\# \text { of SMES } 1)+2 *(\# \text { of SMES } 2)+1 *(\# \text { of SMES } 3)}{\text { Total number of SMEs }}
$$

Where:

SMEs 1: is the group of those who weighted the requirement's importance as high.

SMEs 2: is the group of those who weighted the requirement's importance as medium.

SMEs 3: is the group of those who weighted the requirement's importance as low.

The 2,000 tpd large-size FPU was selected as the base case. The 200 - 500 tpd mediumsize unit is compared to the large FPU, and the SMEs were asked if the medium unit would have a big advantage, a median advantage, would perform the same, would be at a median disadvantage, or a big disadvantage. The SMEs then repeated the process comparing the 50 tpd small-size units to the large unit. The coding to convert the qualitative responses from the SMEs to a qualitative scale is shown in Table 6.1. During this data collection process, SMEs were also able to provide comments on any additional requirement during the interview.

Table 6.1. Code for Requirements' Evaluation Assessment Scores

\begin{tabular}{|c|c|c|c|c|c|}
\hline The code: & $\begin{array}{c}\text { Big } \\
\text { advantage }\end{array}$ & $\begin{array}{c}\text { Median } \\
\text { advantage }\end{array}$ & Same & $\begin{array}{c}\text { Median } \\
\text { disadvantage }\end{array}$ & $\begin{array}{c}\text { Big } \\
\text { disadvantage }\end{array}$ \\
\hline
\end{tabular}

Scores for the medium and small FPUs were calculated by summing the products of the requirements' weights and scores. The large FPU score is " 0 " because it is the base case. Higher scores for the other FPUs indicate a better fit to stakeholders' requirements and lower scores indicate a poorer fit. 
Tasks four and five were the integration of two alternative DM tools (WSM and TOPSIS) with the Pugh Concept Selection Matrix. This included biofuel production requirements with their associated weights, three fast pyrolysis unit alternatives, and a comparison of the performance of the medium and small size fast pyrolysis unit alternatives in comparison to the base case large fast pyrolysis unit. The purpose of this analysis was to determine if any insights could be gained by applying additional DM tools to the problem of determining which specific FPU can be recommended.

The final work was to compare and contrast the results obtained from the original and the two new DM tools application.

\section{Results}

\section{DM tools investigation}

An investigation of MCDA tools, as DM tools, answered the first two research questions. The first question was to identify the DM tools that were applied to energy decision problems. The second question was concerned with finding the advantages and limitations of these DM tools. Table 6.2 summarizes the results of this investigation.

Table 6. 2. DM tools definitions, advantages, and disadvantages.

\begin{tabular}{|c|c|c|c|c|}
\hline 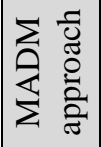 & Definition & Advantages & Disadvantages & References \\
\hline$\sum_{i 2}$ & $\begin{array}{l}\text { - Alternative score }=\text { the } \\
\text { summation of the } \\
\text { products of each } \\
\text { criterion performance } \\
\text { measure and associated } \\
\text { importance weight } \\
\text { - Scores enable the } \\
\text { comparison and ranking } \\
\text { of alternatives }\end{array}$ & $\begin{array}{l}\text { - Simple technique; easy to } \\
\text { use and understand } \\
\text { - Easy to explain to } \\
\text { stakeholders }\end{array}$ & $\begin{array}{l}\text { - Performance measures } \\
\text { must be measurable, } \\
\text { comparable, and } \\
\text { expressed in the same } \\
\text { units } \\
\text { - All criteria are assumed } \\
\text { to be evaluated } \\
\text { independently }\end{array}$ & $\begin{array}{l}\text { (Triantaphyllou } \\
\text { \& Baig, 2005; } \\
\text { Componation \& } \\
\text { Nicholis, 2011) }\end{array}$ \\
\hline
\end{tabular}




\begin{tabular}{|c|c|c|c|c|}
\hline 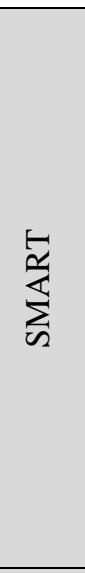 & $\begin{array}{l}\text { - An extension of WSM } \\
\text { - Allows the decision } \\
\text { maker to use value } \\
\text { functions to assess } \\
\text { alternatives' } \\
\text { performance against } \\
\text { specific criteria }\end{array}$ & $\begin{array}{l}\text { - Enables direct comparisons } \\
\text { among performance } \\
\text { measures that have different } \\
\text { units and/or different } \\
\text { direction of improvement } \\
\text { - The value functions can be } \\
\text { tailored to meet specific } \\
\text { decision maker } \\
\text { requirements and } \\
\text { preferences } \\
\text { - The graphical presentation } \\
\text { of the results encourage } \\
\text { decision maker to discuss } \\
\text { the results }\end{array}$ & $\begin{array}{l}\text { - More complicated than } \\
\text { WSM } \\
\text { - Data demands can be } \\
\text { significant } \\
\text { - The process of defining } \\
\text { the value functions is } \\
\text { complex and can be } \\
\text { difficult to explain to } \\
\text { stakeholders } \\
\text { - Does not report the best } \\
\text { alternative }\end{array}$ & $\begin{array}{c}\text { (Goodwin } \\
\text { \&Wright, 2004; } \\
\text { Oyetunji \& } \\
\text { Anderson, 2006; } \\
\text { Goodwin \& Wright, } \\
\text { 2009; Componation } \\
\text { \& Nicholis, 2011) }\end{array}$ \\
\hline$\frac{1}{4}$ & $\begin{array}{l}\text { - A quantitative } \\
\text { comparison method } \\
\text { based on pair-wise } \\
\text { comparisons of decision } \\
\text { criteria }\end{array}$ & $\begin{array}{l}\text { - Suitability for the } \\
\text { evaluation of qualitative } \\
\text { criteria } \\
\text { - Ability to measure decision } \\
\text { maker consistency } \\
\text { explicitly } \\
\text { - Compare each to criterion } \\
\text { together and help to } \\
\text { understand the relation } \\
\text { between criteria }\end{array}$ & $\begin{array}{l}\text { - Can be too involved and } \\
\text { time consuming for } \\
\text { decisions involving } \\
\text { many criteria and } \\
\text { stakeholders } \\
\text { - No absolute measures of } \\
\text { performance; all are } \\
\text { relative } \\
\text { - Hard to explain to } \\
\text { stakeholders }\end{array}$ & $\begin{array}{l}\text { (Saaty, 1980; } \\
\text { Saaty \& Salmanca- } \\
\text { Buentello, 1994; } \\
\text { Belton \& Stewart, } \\
\text { 2002; Componation } \\
\text { \& Nicholis, 2011) }\end{array}$ \\
\hline $\begin{array}{l}\tilde{n} \\
\tilde{\tilde{D}} \\
\tilde{0}\end{array}$ & $\begin{array}{l}\text { - A method that selects } \\
\text { the alternative that is } \\
\text { closest to the ideal } \\
\text { solution and furthest } \\
\text { from the worst-case } \\
\text { solution }\end{array}$ & $\begin{array}{l}\text { - Intuitive underlying concept } \\
\text { - Provides a good } \\
\text { understanding of the } \\
\text { problem }\end{array}$ & $\begin{array}{l}\text { - Complex evaluation } \\
\text { procedure may be } \\
\text { problematic for decision } \\
\text { makers lacking } \\
\text { experience with the use } \\
\text { of quantitative analysis }\end{array}$ & $\begin{array}{l}\text { (Yoon \& Hwang, } \\
\text { 1995; Tong, Wang, } \\
\text { \& Chen, 2004; } \\
\text { Componation \& } \\
\text { Nicholis, 2011) }\end{array}$ \\
\hline 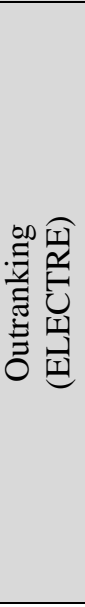 & $\begin{array}{l}\text { - Methods in which the } \\
\text { performance of } \\
\text { alternatives in } \\
\text { comparison to each } \\
\text { other, rather than } \\
\text { achieving a final } \\
\text { ranking of alternatives. } \\
\text { - Family of methods } \\
\text { - Examples: ELECTRE I } \\
\text { \& ELECTRE II, } \\
\text { ELECTRE III }\end{array}$ & $\begin{array}{l}\text { - Allows incomplete data sets } \\
\text { to be used } \\
\text { - Allows for quantitative and } \\
\text { qualitative criteria } \\
\text { - Provides a good } \\
\text { understanding of the } \\
\text { problem }\end{array}$ & $\begin{array}{l}\text { - A final ranking of } \\
\text { alternative preferences } \\
\text { is not reached } \\
\text { - Does not provide the } \\
\text { best alternative } \\
\text { - Stakeholders may find } \\
\text { the method to be too } \\
\text { technically difficult to } \\
\text { fully understand the } \\
\text { approach } \\
\text { - Many non-intuitive } \\
\text { inputs are required } \\
\text { - Not comfortable to use } \\
\text { - Not easy to explain to } \\
\text { stakeholders }\end{array}$ & $\begin{array}{r}\text { (Roy, Present, \& } \\
\text { Sithol, 1986; Belton } \\
\text { \& Stewart, 2002; } \\
\text { Wang et al., 2009) }\end{array}$ \\
\hline
\end{tabular}




\begin{tabular}{|c|c|c|c|c|}
\hline 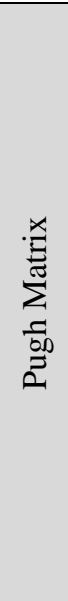 & $\begin{array}{l}\text { - It is a paired comparison } \\
\text { analysis } \\
\text { - Could compare } \\
\text { alternatives or criteria }\end{array}$ & $\begin{array}{l}\text { - Easy to use } \\
\text { - Does not require complex } \\
\text { mathematical formulas } \\
\text { - Can be used when there is } \\
\text { little or no data available } \\
\text { - Works well when } \\
\text { stakeholders are challenged } \\
\text { with a problem that has } \\
\text { multiple perspectives and } \\
\text { requirements } \\
\text { - Useful for decisions that } \\
\text { deal with quantitative and } \\
\text { qualitative data, in addition } \\
\text { to uncertain environments } \\
\text { and risky situations }\end{array}$ & $\begin{array}{l}\text { - Not a strong tool to } \\
\text { provide a final decision } \\
\text { for a complex problem }\end{array}$ & $\begin{array}{c}\text { (Pugh, 1991; } \\
\text { Min, 1994; } \\
\text { Cervone, 2009a; } \\
\text { Cervone, 2009b) }\end{array}$ \\
\hline
\end{tabular}

WSM is the simplest and most commonly used DM tool (Triantaphyllou \& Baig, 2005; Theodorou, Florides, \& Tassou, 2010). With this approach, the score $P_{i}$ for an alternative $A_{i}$ is determined by summing the products of the criteria importance weights $w_{j}$ and the performance scores $a_{i j}$ for that alternative in relation to the criteria $C_{j}$. The performance scores $a_{i j}$ are such that higher values are better, and the same units are used for all criteria; therefore, the alternative with the largest cumulative score $P_{i}$ is determined to be the most preferred choice. With this method, the higher the value of $w_{j}$ is, the more important its associated criterion $C_{j}$ is assumed to be. The values of $w_{j}$ are typically normalized so that they sum to one.

WSM has been applied to the complex decision problem of determining the best biofuel conversion pathway, where a "pathway" is characterized by the types of biomass/biofuel production methods used, as well as the biomass/biofuel logistics and distribution schemes. In their analysis of a biodiesel conversion pathway, Perimenis et al. (2011) derive performance measures within four categories of assessment criteria: technical, economic, environmental, and social. Each of the measures is then converted to a constructed scale with values from 1 to 5 (where 5 is best), and weights are determined via a simple comparison matrix in which the relative importance of each criterion was determined by stakeholders. For each alternative, each 
performance measure score is multiplied by its respective weight and the results are summed to give a final score. Using these scores, the pathway alternatives can be compared and ranked (Perimenis et al., 2011).

The SMART method is an extension of WSM that allows the decision maker to use value functions to determine each performance score $a_{i j}$. The value functions map the range of values for each performance measure to a common dimensionless scale, where the most-preferred variable is assigned a value of 100 , the least-preferred variable is assigned a value of 0 , and additional alternatives are given values between 0 and 100 (Goodwin \& Wright, 2009). The value functions can be tailored to match the requirements and preferences of decision makers and stakeholders, and as a result, the functions are often non-linear (Componation et al., 2013).

In their review of MCDA applications to sustainable energy decision problems, Theodorou et al. (2010) reported that SMART has not been widely used to analyze energy planning decisions and suggest that this may be because the determination of the value functions is data-intensive and complex, and decision makers and stakeholders may find the mapping of performance measures to value functions to be too abstract. The literature review did not identify any applications of SMART to biofuel/biomass decision problems. In the more general domain of renewable energy decision making, Jones et al. (1990) used SMART to evaluate five national energy policy portfolios using 41 performance measures, which were divided into five categories: economic, environmental, political, social, and technical. The portfolios contained a variety of energy sources (e.g., fossil fuels, nuclear, solar), as well as the option to implement energy conservation measures (Jones, Hope, \& Hughes, 1990).

AHP is a popular MCDA method developed by Thomas Saaty (1980). AHP is similar to the WSM and SMART in that all of these methods use an additive preference function to 
evaluate alternatives (Belton \& Stewart, 2002). However, rather than using absolute measures of performance and value functions, AHP is based on a series of pairwise comparisons of the decision criteria, in which all individual criteria must be paired and compared against each other. The decision maker uses a numerical scale ( 1 to 9$)$ to compare the criteria/alternative $p$ to $q$, where low values represent weak or no preference for $\mathrm{p}$ over $\mathrm{q}$, and high values indicate strong or absolute preference for $p$ over $q$. Once all pairs of alternatives have been compared, the resulting values are compiled in a matrix. The eigenvector corresponding to the maximum eigenvalue of the matrix is determined through a process of iterative calculations, and this vector of scores is normalized to sum to one, which results in a final list of relative preferences. The decision maker's consistency throughout all pairwise comparisons can be measured explicitly by calculating a consistency index.

In their reviews, both Zhou et al. (2006) and Theodourou et al. (2010) identified AHP as the most widely used MCDA method for renewable energy planning. Zhou et al. (2006) suggested that AHP's popularity is due to its simplicity, ease of understanding, and suitability for the evaluation of qualitative criteria. However, Theodourou et al. (2010) noted that AHP is best applied to decisions in which there are relatively few criteria and decision makers, indicating that the pairwise comparison process can make the analysis of large problems overly cumbersome. Kurka (2013) applied AHP to a decision problem in which four alternative scenarios for regional bioenergy production were compared. Each scenario differed in terms of energy production scale, number of facilities, degree of facility centralization, and type/source of biomass. Nine criteria were used to compare the alternatives, and these criteria were divided into four categories: economic, environmental, technical, and social. The model was applied to a case study in Scotland, and results were analyzed for sensitivity to changes in weights (Kurka, 2013). 
TOPSIS is an MCDA technique developed by Yoon and Hwang (1995). The concept of this method is that the best alternative should be near the theoretical positive ideal solution and far away from the theoretical negative solution. The positive ideal solution has the best score from each requirement (i.e., a theoretical solution that has an optimal performance score for all criteria), whereas the negative ideal is the one with worst scores form all requirement (Wang et al., 2008; Wang et al., 2009). To calculate the "distances" between actual and ideal performance scores, the Euclidean formula is used. The weighted distance $d_{i}$ between an alternative $A_{i}$ and the ideal solution $A^{*}$ is the sum of the squared distances between the actual performance score $a_{i j}$ and the ideal score $a_{j} *$ for each criteria $C_{j}$, multiplied by the squared weight $w_{j}$ of criteria $C_{j}$ (Wang et al., 2009). The distances $d_{i}^{-}$between actual scores and worst-case scores are calculated similarly. The relative closeness of each alternative $A_{i}$ to the ideal alternative is then determined by calculating the ratio of the negative distances to the positive distances.

In their review of applications of MCDA methods to energy decisions, Zhou et al. (2006) reported that TOPSIS has not been widely implemented. The literature review did not identify any existing applications of TOPSIS to decision problems in the bioenergy domain. Yang and Chen (1989) used TOPSIS to extend a single-objective cost minimizing decision analysis on how much nuclear energy production capacity should be added to Taiwan's portfolio of energy sources, as well as the timeline for this increase, to an MCDA. The new analysis included four categories of criteria: cost, environmental considerations, vulnerability of fuel supply, and risk of disaster. TOPSIS was used in conjunction with optimization methods to evaluate the optimal energy portfolio for five different scenarios (Yang \& Chen, 1989).

The outranking methods (i.e., ELECTRE and PROMETHEE) are fundamentally different from other MCDA methods in that there is no underlying aggregate value function, and the final 
output of an analysis is not a specific value for each alternative, but rather an "outranking relation", or matrix, that shows the relationships between alternatives (Belton \& Stewart, Multiple Criteria Decision Analysis: An Integrated Approach, 2002). This output enables decision makers to determine whether a given alternative $A_{i}$ outperforms (i.e., outranks) alternative $A_{j}$. The emphasis is on understanding the performance of alternatives in relationship to each other rather than resulting in a final ranking (Componation et al., 2013).

Outranking methods, especially ELECTRE III and PROMETHEE, are widely used in renewable energy planning, particularly in the domain of bioenergy (Beccali, Cellura, \& Mistretta, 2003). In their review of MCDA methods as applied to decisions in bioenergy systems, Scott et al. (2012) identified nine papers in the literature that used outranking methods.

In summary, this investigation of DM tools indicated that the two most-commonly applied DM tools in biofuel decision problems are AHP and ELECTRE (Beccali et al., 2003; Roy, Present, \& Sithol, 1986). In addition, WSM was commonly mentioned in previous biofuel decisions as an easy DM tool (Triantaphyllou \& Baig, 2005; Perimenis et al., 2011). Other DM tools, such as SMART and TOPSIS, are not widely adopted in energy modeling (Zhou et al., 2006; Theodorou et al., 2010). Similarly, Pugh Matrix has not been applied to decisions in the renewable energy domain, other than one report that mentioned the use of this tool for a decision about biodiesel produced from different microalgae strains (Coelho, Dutta, \& Silva, 2013). Table 6.3 summarizes these results. 
Table 6.3. DM tools application in energy decisions and biofuel industry

\begin{tabular}{|c|c|c|}
\hline 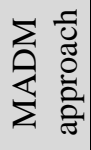 & Energy \& biofuel industry applications & References \\
\hline$\sum_{n}$ & $\begin{array}{l}\text { - Used to decide among multiple biofuel conversion pathways (i.e., } \\
\text { sequences of biomass/biofuel production and distribution alternatives), } \\
\text { considering technical, economic, environmental, and social criteria }\end{array}$ & (Perimenis et al., 2011) \\
\hline 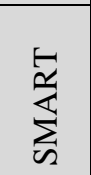 & $\begin{array}{l}\text { - Applied to the multi-attribute problem of determining which energy policy } \\
\text { should be selected for the UK, given five different portfolios of energy } \\
\text { sources }\end{array}$ & (Jones et al., 1990) \\
\hline 是 & $\begin{array}{l}\text { - Widely employed to sustainable energy DM: social, economic, agriculture, } \\
\text { industrial, ecological, and biological systems (energy systems) } \\
\text { - Applied on decisions for: } \\
\begin{aligned} & \circ \text { Oil and gas } \\
& \circ \text { Renewable energy and energy in general } \\
& \circ \text { Energy planning and policy analysis } \\
& \circ \text { Site selection } \\
& \circ \text { Production } \\
& 0 \text { Environmental impact assessment } \\
& \text { - Used to determine which of four regional bioenergy production scenarios } \\
& \text { should be implement, where each scenario varied in terms of scale, } \\
& \text { location/degree of centralization of facilities, and type/source of biomass }\end{aligned}\end{array}$ & $\begin{array}{l}\text { (Gwo-Hshiung, Tzay-an, \& } \\
\text { Chien-Yuan, 1992; Huang, } \\
\text { Poh, \& Ang, 1995; Wang et } \\
\text { al., 2009; Ho, Xu, Dey, 2010; } \\
\text { Kurka, 2013) }\end{array}$ \\
\hline$\frac{n}{\tilde{\vartheta}}$ & $\begin{array}{l}\text { - Applied to decision problem of when/by how much to increase nuclear } \\
\text { energy production capacity, relative to the available capacity of other } \\
\text { energy sources }\end{array}$ & (Yang \& Chen, 1989) \\
\hline 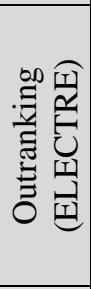 & $\begin{array}{l}\text { Applied on decisions for: } \\
\text { - Feedstock sources } \\
\text { - Biofuel processing technology selection } \\
\text { - Comparison of biofuels/biomass with other energy sources to determine } \\
\text { optimal source or mix of sources } \\
\text { - Biofuel selection in vehicles }\end{array}$ & $\begin{array}{l}\text { (Georgopoulou et al., 1997; } \\
\text { Beccali et al., 2003; } \\
\text { Mohamadabi et al., 2009) }\end{array}$ \\
\hline 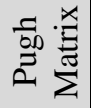 & - Biodiesel produced from different microalgae strains & (Coelho, Dutta, \& Silva, 2013) \\
\hline
\end{tabular}

\section{DM Integrated Approaches Implementation of the FPUs' Decision Problem}

For the purpose of this study, we decided to solve the FPU size selection problem using a simple and straightforward DM tool, and then using a more complicated DM tool to determine whether the outcomes were different and to gain insights into the value of approaching the same problem using two different tools. In addition, we decided to solve the problem using one tool 
that has already been used in this field and one that has not previously been applied in this field before (or had limited application).

Although AHP and ELECTRE have already been applied widely in this field, TOPSIS is a tool that has limited application in this area. Moreover, TOPSIS is not classified as an easy and straightforward DM tool. Therefore, we selected this tool as the one representing the complicated tool and the one that has limited application in this field.

WSM is classified as the easiest tool for DM, and it has frequently been applied in this field. Therefore, we selected this tool as the one representing the easy tool and the one that has been applied in this field previously.

The third research question looks at whether an individual or a set of DM tools would provide better insight into the complex problem of biofuel production, and the fourth research question was concerned with which FPU is recommended for biofuel energy production based on stakeholder perceptions. First, prior work was used to define the problem and collect the data. Then, the problem was solved using WSM and TOPSIS. Finally, the results from the two DM tools in addition to the result from the prior work will be discussed and compared.

The process of problem identification that was performed in prior work is summarized in the following steps:

- Decision maker identification: A total of 36 unique BPS were identified in the literature (Fawzy \& Componation, 2014). Further study grouped the BPS into five perspectives: Economic, environmental, technical, social, and legal (Fawzy \& Componation, 2015a). The sample selected to collect the data is a group of SMEs in bioenergy where each of the five stakeholders' perspectives is represented by at least one out of the responding 10 SMEs. 
- Requirements identification: 18 requirements are selected to be the decision criteria as shown in Table 6.4 (Fawzy \& Componation, 2015a).

- Decision goals: The objective was to determine the optimal sizing of an FPU that reduces capital costs, investment risk, and is more responsive to changes in energy demands (Fawzy \& Componation, 2015b). Table 6.4 summarizes the desired direction of improvement for each requirement (Fawzy \& Componation, 2015a).

Table 6.4. Decision criteria and goals

\begin{tabular}{|c|c|}
\hline Requirements & Goals \\
\hline $\mathrm{CO}_{2}$ emission & (-) Minimize \\
\hline Resources saving & (-) Minimize \\
\hline Design cost & (-) Minimize \\
\hline Capital cost (Including Equipment) & (-) Minimize \\
\hline Equipment cost & (-) Minimize \\
\hline Labor cost & (-) Minimize \\
\hline Production cost in the facility & (-) Minimize \\
\hline Materials cost (Feedstock) & (-) Minimize \\
\hline Transportation cost for feedstock & (-) Minimize \\
\hline Response to market \& policy & (+) Maximize \\
\hline Storage cost for feedstock & (-) Minimize \\
\hline Storage cost for produced biofuel & (-) Minimize \\
\hline Annual investments (Maintenance) & (-) Minimize \\
\hline Energy saving & (+) Maximize \\
\hline Biomass availability (Now) & (+) Maximize \\
\hline Operation efficiency & (+) Maximize \\
\hline Policy \& regulations & (+) Maximize \\
\hline Number of jobs offered & (+) Maximize \\
\hline
\end{tabular}

- Identify the FPUs sizes: Three FPU sizes were identified as the solution alternatives (Fawzy \& Componation, 2015a). These three unit sizes with their capacities of biomass consumption are shown in Table 6.5 .

Table 6.5. Decision alternatives

\begin{tabular}{||c|c|c||}
\hline$\#$ & Alternatives & Consumption capacities \\
\hline 1 & Large FPU & Consumes $\geq 2000$ tpd of biomass \\
\hline 2 & Medium FPU & Consumes between $200-500$ tpd of biomass \\
\hline 3 & Small FPU & Consumes $\leq 50$ tpd of biomass \\
\hline
\end{tabular}


- Create the DM matrix: The last step before data collection was to frame the problem. Table 6.6 shows the matrix that was structured for the problem. The first column in the matrix contains the list of 18 criteria. The second column stores the average values of the SME evaluations for weights on each requirement, while the last three columns store the average values of all SME evaluations for each FPU at each requirement using the Pugh Concept

\section{Selection Matrix.}

Table 6.6. Decision criteria and goals

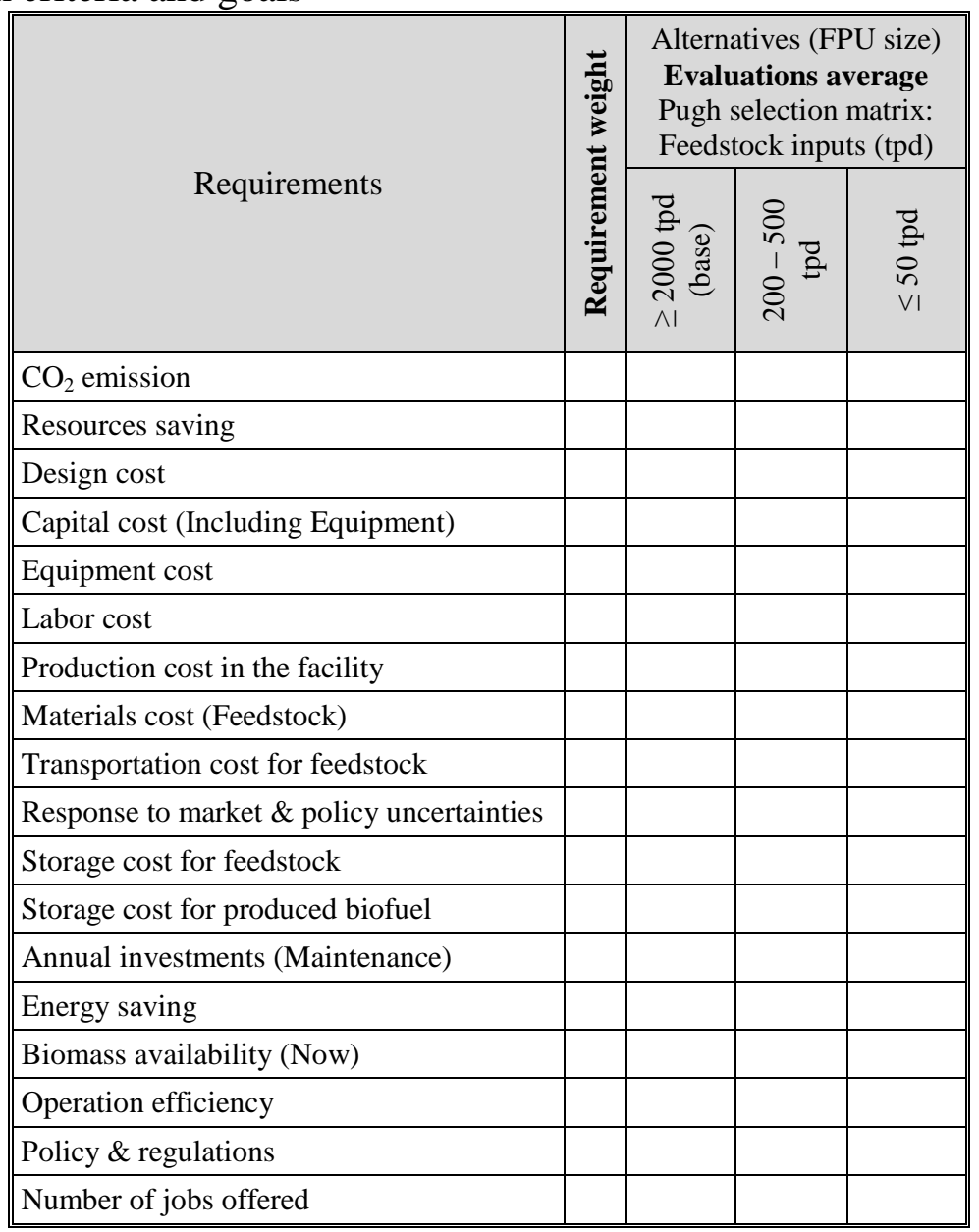

SME interviews and data collection were conducted through two steps. First, SMEs evaluated the requirements; second, SMEs evaluated the alternatives. 
- Evaluate the identified requirements: The SMEs placed each requirement in one of three groups: High importance, medium importance, and low importance. Then, the requirements weights were calculated using Equation 6.1; results are shown in Table 6.7. Next, the raw data from performance of each FPU was compared against the base case. Finally, an average value of the ten SMEs was obtained using their evaluations for each requirement using Equation 6.2 (results given in Table 6.8).

Table 6.7. Requirements' evaluations and weights

\begin{tabular}{|c|c|c|c|c|c|}
\hline \multirow[b]{2}{*}{ Requirements } & \multicolumn{4}{|c|}{$\begin{array}{l}\text { SMEs evaluation } \\
\text { for requirements' } \\
\text { importance }\end{array}$} & \multirow{2}{*}{ 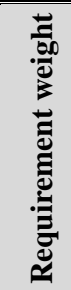 } \\
\hline & $\mathrm{H}$ & M & $\mathrm{L}$ & 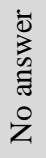 & \\
\hline $\mathrm{CO}_{2}$ emission & 4 & 4 & 2 & 0 & 2.20 \\
\hline Resources saving & 5 & 4 & 1 & 0 & 2.40 \\
\hline Design cost & 4 & 4 & 2 & 0 & 2.20 \\
\hline Capital cost (Including Equipment) & 9 & 1 & 0 & 0 & 2.90 \\
\hline Equipment cost & 7 & 2 & 1 & 0 & 2.60 \\
\hline Labor cost & 2 & 2 & 6 & 0 & 1.60 \\
\hline Production cost in the facility & 7 & 3 & 0 & 0 & 2.70 \\
\hline Materials cost (Feedstock) & 7 & 3 & 0 & 0 & 2.70 \\
\hline Transportation cost for feedstock & 5 & 2 & 2 & 1 & 2.33 \\
\hline Response to market \& policy uncertainties & 7 & 2 & 1 & 0 & 2.60 \\
\hline Storage cost for feedstock & 3 & 5 & 2 & 0 & 2.10 \\
\hline Storage cost for produced biofuel & 4 & 1 & 4 & 1 & 2.00 \\
\hline Annual investments (Maintenance) & 6 & 4 & 0 & 0 & 2.60 \\
\hline Energy saving & 3 & 6 & 1 & 0 & 2.20 \\
\hline Biomass availability (Now) & 6 & 3 & 1 & 0 & 2.50 \\
\hline Operation efficiency & 4 & 6 & 0 & 0 & 2.40 \\
\hline Policy \& regulations & 6 & 4 & 0 & 0 & 2.60 \\
\hline Number of jobs offered & 2 & 4 & 4 & 0 & 1.80 \\
\hline
\end{tabular}

- Evaluate FPU sizes using Pugh Concept Selected Matrix: The SMEs evaluated the small and medium unit sizes in comparison with the large unit. Each SME evaluated the FPUs against 
each individual requirement using the Pugh Concept Selection matrix as a DM tool. Then, the average value of the ten SMEs was calculated using Equation 6.2 as shown in Table 6.8.

$$
\text { Avg. of requirement's scores }=\frac{\sum_{i=1}^{10} S M E_{i}}{\text { Total number of } S M E S}
$$

Table 6.8. SMEs average evaluations using Pugh Concept Selection Matrix.

\begin{tabular}{|c|c|c|c|c|}
\hline \multirow{2}{*}{ Requirements } & \multirow{2}{*}{ 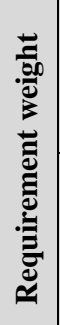 } & \multicolumn{3}{|c|}{$\begin{array}{l}\text { Alternatives (FPU size) } \\
\text { Evaluations average } \\
\text { Pugh selection matrix: } \\
\text { Feedstock inputs (tpd) }\end{array}$} \\
\hline & & 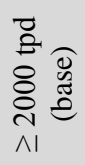 & $\begin{array}{l}8 \\
n^{2} \\
1 \\
\overbrace{1}^{4}\end{array}$ & $\begin{array}{l}\vec{D} \\
\dot{2} \\
0 \\
0 \\
\text { vi }\end{array}$ \\
\hline $\mathrm{CO}_{2}$ emission & 2.2 & 0 & 0.111 & -0.444 \\
\hline Resources saving & 2.4 & 0 & 0.125 & -0.125 \\
\hline Design cost & 2.2 & 0 & 0.333 & 0.778 \\
\hline Capital cost (Including Equipment) & 2.9 & 0 & -0.700 & -1.300 \\
\hline Equipment cost & 2.6 & 0 & -0.5 & -1.200 \\
\hline Labor cost & 1.6 & 0 & -0.7 & -1.100 \\
\hline Production cost in the facility & 2.7 & 0 & -0.444 & -0.889 \\
\hline Materials cost (Feedstock) & 2.7 & 0 & 0.400 & 0.700 \\
\hline Transportation cost for feedstock & 2.3 & 0 & 1.111 & 2.000 \\
\hline Response to market \& policy uncertainties & 2.6 & 0 & 0.556 & 0.889 \\
\hline Storage cost for feedstock & 2.1 & 0 & 0.500 & 0.900 \\
\hline Storage cost for produced biofuel & 2.0 & 0 & -0.222 & -0.333 \\
\hline Annual investments (Maintenance) & 2.6 & 0 & 0.300 & 0.100 \\
\hline Energy saving & 2.2 & 0 & -0.500 & -0.700 \\
\hline Biomass availability (Now) & 2.5 & 0 & 0.800 & 1.400 \\
\hline Operation efficiency & 2.4 & 0 & -0.500 & -0.600 \\
\hline Policy \& regulations & 2.6 & 0 & -0.125 & 0.250 \\
\hline Number of jobs offered & 1.8 & 0 & 1.000 & 1.700 \\
\hline
\end{tabular}

The fourth and fifth tasks in this research were to solve the problem using two DM

tools: WSM and TOPSIS. The inputs for these two tools were the collected data from previous tasks in Pugh Matrix (Table 6.8).

For solving the problem using WSM, Equation 6.3 was used for calculating the requirements scores for each FPU (see Table 6.9 for a summary of the results). 
Requirement's score $=$ Avg. of requirement's scores $*$ Requirement's weight

Then, the total score for each FPU was calculated using Equation 6.4 (see Table 6.9).

$$
\text { FPU's score }=\sum_{i=1}^{18}\left(\text { Requirement }_{i} \text { score }\right)
$$

Table 6.9. Requirements and alternatives final weighted scores

\begin{tabular}{|c|c|c|c|c|c|c|c|}
\hline \multirow{2}{*}{ Requirements } & \multirow{2}{*}{ 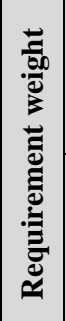 } & \multicolumn{3}{|c|}{$\begin{array}{l}\text { Alternatives (FPU size) } \\
\text { Evaluations average } \\
\text { Pugh selection matrix: } \\
\text { Feedstock inputs (tpd) }\end{array}$} & \multicolumn{3}{|c|}{$\begin{array}{l}\text { Alternatives (FPU size) } \\
\text { Weighted scores } \\
\text { Pugh selection matrix: } \\
\text { Feedstock inputs (tpd) }\end{array}$} \\
\hline & & 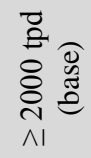 & 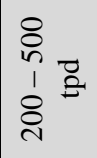 & 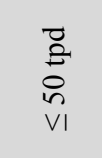 & 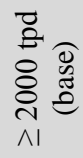 & 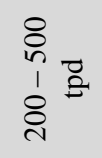 & $\begin{array}{l}\text { D } \\
\text { ह } \\
0 \\
\text { VI }\end{array}$ \\
\hline $\mathrm{CO}_{2}$ emission & 2.2 & 0 & 0.111 & -0.444 & 0 & 0.244 & -0.978 \\
\hline Resources saving & 2.4 & 0 & 0.125 & -0.125 & 0 & 0.300 & -0.300 \\
\hline Design cost & 2.2 & 0 & 0.333 & 0.778 & 0 & 0.733 & 1.711 \\
\hline Capital cost (Including Equipment) & 2.9 & 0 & -0.700 & -1.300 & 0 & -2.030 & -3.770 \\
\hline Equipment cost & 2.6 & 0 & -0.5 & -1.200 & 0 & -1.300 & -3.120 \\
\hline Labor cost & 1.6 & 0 & -0.7 & -1.100 & 0 & -1.120 & -1.760 \\
\hline Production cost in the facility & 2.7 & 0 & -0.444 & -0.889 & 0 & -1.200 & -2.400 \\
\hline Materials cost (Feedstock) & 2.7 & 0 & 0.400 & 0.700 & 0 & 1.080 & 1.890 \\
\hline Transportation cost for feedstock & 2.3 & 0 & 1.111 & 2.000 & 0 & 2.593 & 4.667 \\
\hline Response to market \& policy uncertainties & 2.6 & 0 & 0.556 & 0.889 & 0 & 1.444 & 2.311 \\
\hline Storage cost for feedstock & 2.1 & 0 & 0.500 & 0.900 & 0 & 1.050 & 1.890 \\
\hline Storage cost for produced biofuel & 2.0 & 0 & -0.222 & -0.333 & 0 & -0.444 & -0.667 \\
\hline Annual investments (Maintenance) & 2.6 & 0 & 0.300 & 0.100 & 0 & 0.780 & 0.260 \\
\hline Energy saving & 2.2 & 0 & -0.500 & -0.700 & 0 & -1.100 & -1.540 \\
\hline Biomass availability (Now) & 2.5 & 0 & 0.800 & 1.400 & 0 & 2.000 & 3.500 \\
\hline Operation efficiency & 2.4 & 0 & -0.500 & -0.600 & 0 & -1.200 & -1.440 \\
\hline Policy \& regulations & 2.6 & 0 & -0.125 & 0.250 & 0 & -0.325 & 0.650 \\
\hline Number of jobs offered & 1.8 & 0 & 1.000 & 1.700 & 0 & 1.800 & 3.060 \\
\hline \multicolumn{5}{|l|}{ Final Scores: } & 0 & 3.305 & 3.964 \\
\hline
\end{tabular}

From Table 6.9 we see that because the large FPU is the base case for Pugh Concept

Selection Matrix, all the requirements scores for this FPU are equal to zero. The medium FPU

has a total score equal to 3.305. The small FPU has the highest total score, equal to 3.964.

The last step in WSM was to normalize the requirements weights and recalculate the alternatives scores to select the best alternative. The weights were normalized using Equation 
6.5. After that, the model was re-run, and Equation 6.4 was used to calculate the alternatives' total scores. Finally, the FPU with the highest score is selected as the best alternative (Table 6.10).

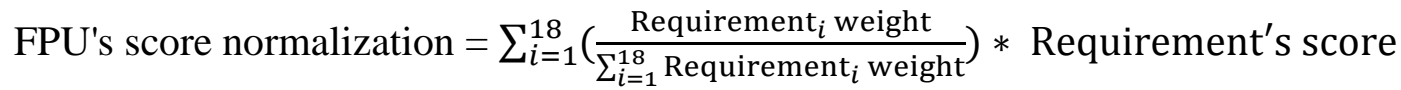

Table 6.10. Requirements and alternatives final normalized scores

\begin{tabular}{|c|c|c|c|c|}
\hline \multirow{2}{*}{ Requirements } & \multirow{2}{*}{ 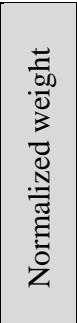 } & \multicolumn{3}{|c|}{$\begin{array}{l}\text { Alternatives (FPU size) } \\
\text { Normalized scores } \\
\text { Pugh selection matrix: } \\
\text { Feedstock inputs (tpd) }\end{array}$} \\
\hline & & 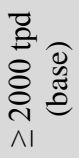 & $\begin{array}{l}\delta_{0}^{8} \\
\overbrace{1}^{1} \\
0\end{array}$ & $\begin{array}{l}\text { है } \\
0 \\
i n \\
\text { VI }\end{array}$ \\
\hline $\mathrm{CO}_{2}$ emission & 0.06 & 0 & 0.006 & -0.023 \\
\hline Resources saving & 0.06 & 0 & 0.007 & -0.007 \\
\hline Design cost & 0.06 & 0 & 0.017 & 0.040 \\
\hline Capital cost (Including Equipment) & 0.08 & 0 & -0.048 & -0.089 \\
\hline Equipment cost & 0.06 & 0 & -0.031 & -0.074 \\
\hline Labor cost & 0.04 & 0 & -0.026 & -0.042 \\
\hline Production cost in the facility & 0.07 & 0 & -0.028 & -0.057 \\
\hline Materials cost (Feedstock) & 0.07 & 0 & 0.025 & 0.045 \\
\hline Transportation cost for feedstock & 0.06 & 0 & 0.061 & 0.110 \\
\hline Response to market $\&$ policy uncertainties & 0.07 & 0 & 0.034 & 0.054 \\
\hline Storage cost for feedstock & 0.05 & 0 & 0.025 & 0.045 \\
\hline Storage cost for produced biofuel & 0.05 & 0 & -0.010 & -0.016 \\
\hline Annual investments (Maintenance) & 0.07 & 0 & 0.018 & 0.006 \\
\hline Energy saving & 0.06 & 0 & -0.026 & -0.036 \\
\hline Biomass availability (Now) & 0.07 & 0 & 0.047 & 0.082 \\
\hline Operation efficiency & 0.06 & 0 & -0.028 & -0.034 \\
\hline Policy \& regulations & 0.07 & 0 & -0.008 & 0.015 \\
\hline Number of jobs offered & 0.05 & 0 & 0.042 & 0.072 \\
\hline Final Scores: & 1 & 0 & 0.078 & 0.093 \\
\hline
\end{tabular}

As shown in Table 6.10, the large FPU has a total score equal to zero. The medium FPU has a total score equal to 0.078. Finally, the small FPU has the highest score total equal to 0.093. The small unit is $9.3 \%$ better than the big FPU. In conclusion, by using WSM, the small FPU is recommended as the best alternative that fits the stakeholders' requirements. 
Next, the problem was solved using TOPSIS. Similar to WSM, Equations 6.2 through 6.4 were applied for the TOPSIS method. Tables 6.5 through 6.8 also show the application of Equations 6.2 through 6.4 for the TOPSIS method and the obtained results. Then, both positive and negative ideal solutions are determined respectively (see Table 6.11). Similar to WSM, the total score for each FPU including the positive and negative ideal solutions was calculated using Equation 6.4 (see Table 6.11).

Table 6.11. Positive and negative ideal solutions calculations with all alternatives final scores

\begin{tabular}{|c|c|c|c|c|c|c|c|c|c|c|c|}
\hline \multirow{2}{*}{ Requirements } & \multirow{2}{*}{ 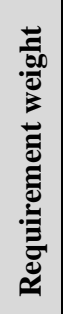 } & \multicolumn{5}{|c|}{$\begin{array}{l}\text { Alternatives (FPU size) } \\
\text { Evaluations average } \\
\text { Pugh selection matrix: } \\
\text { Feedstock inputs (tpd) }\end{array}$} & \multicolumn{5}{|c|}{$\begin{array}{l}\text { Alternatives (FPU size) } \\
\text { Weighted scores } \\
\text { Pugh selection matrix: } \\
\text { Feedstock inputs (tpd) }\end{array}$} \\
\hline & & 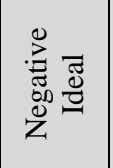 & 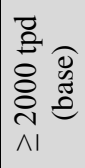 & 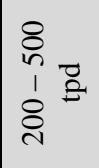 & $\begin{array}{l}\text { D. } \\
\dot{2} \\
0 \\
\stackrel{n}{ } \\
\text { vi }\end{array}$ & 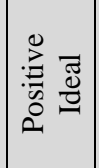 & 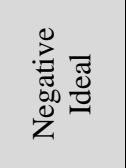 & 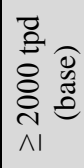 & $\mid \begin{array}{ll}8 \\
0 \\
n \\
1 & 0 \\
8 & 0 \\
0 & 0\end{array}$ & 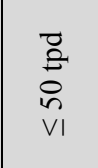 & 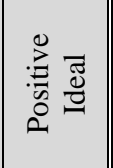 \\
\hline $\mathrm{CO}_{2}$ emission & 2.2 & -0.444 & 0 & 0.111 & -0.444 & 0.111 & -0.978 & 0 & 0.244 & -0.978 & 0.244 \\
\hline Resources saving & 2.4 & -0.125 & 0 & 0.125 & -0.125 & 0.125 & -0.300 & 0 & 0.300 & -0.300 & 0.300 \\
\hline Design cost & 2.2 & 0 & 0 & 0.333 & 0.778 & 0.778 & 0 & 0 & 0.733 & 1.711 & 1.711 \\
\hline Capital cost (Including Equipment) & 2.9 & -1.300 & 0 & -0.700 & -1.300 & \begin{tabular}{|l|}
0 \\
\end{tabular} & -3.770 & 0 & -2.030 & -3.770 & 0 \\
\hline Equipment cost & 2.6 & -1.200 & 0 & -0.5 & -1.200 & 0 & -3.120 & 0 & -1.300 & -3.120 & 0 \\
\hline Labor cost & 1.6 & -1.100 & 0 & -0.7 & -1.100 & 0 & -1.760 & 0 & -1.120 & -1.760 & 0 \\
\hline Production cost in the facility & 2.7 & -0.889 & 0 & -0.444 & -0.889 & 0 & -2.400 & 0 & -1.200 & -2.400 & 0 \\
\hline Materials cost (Feedstock) & 2.7 & 0 & 0 & 0.400 & 0.700 & 0.700 & 0 & 0 & 1.080 & 1.890 & 1.890 \\
\hline Transportation cost for feedstock & 2.3 & 0 & 0 & 1.111 & 2.000 & 2.000 & 0 & 0 & 2.593 & 4.667 & 4.667 \\
\hline Response to market $\&$ policy uncertainties & 2.6 & 0 & 0 & 0.556 & 0.889 & 0.889 & 0 & 0 & 1.444 & 2.311 & 2.311 \\
\hline Storage cost for feedstock & 2.1 & 0 & 0 & 0.500 & 0.900 & 0.900 & 0 & 0 & 1.050 & 1.890 & 1.890 \\
\hline Storage cost for produced biofuel & 2.0 & -0.333 & 0 & -0.222 & -0.333 & 0 & -0.667 & 0 & -0.444 & -0.667 & 0 \\
\hline Annual investments (Maintenance) & 2.6 & 0 & 0 & 0.300 & 0.100 & 0.300 & 0 & 0 & 0.780 & 0.260 & 0.780 \\
\hline Energy saving & 2.2 & -0.700 & 0 & -0.500 & -0.700 & 0 & -1.540 & 0 & -1.100 & -1.540 & 0 \\
\hline Biomass availability (Now) & 2.5 & 0 & 0 & 0.800 & 1.400 & 1.400 & 0 & 0 & 2.000 & 3.500 & 3.500 \\
\hline Operation efficiency & 2.4 & -0.600 & 0 & -0.500 & -0.600 & 0 & -1.440 & 0 & -1.200 & -1.440 & 0 \\
\hline Policy \& regulations & 2.6 & -0.125 & 0 & -0.125 & 0.250 & 0.250 & -0.325 & 0 & -0.325 & 0.650 & 0.650 \\
\hline Number of jobs offered & 1.8 & 0 & 0 & 1.000 & 1.700 & 1.700 & 0 & 0 & 1.800 & 3.060 & 3.060 \\
\hline \multicolumn{7}{|c|}{ Final Scores: } & -16.299 & 0 & 3.305 & 3.964 & 21.003 \\
\hline
\end{tabular}

From Table 6.11 we see that the positive ideal solution has a total score equal to 21.003 and the negative ideal solution has a total score equal to -16.299. The large FPU has a total score 
equal to zero as in previous method, because it is the base case. The medium FPU has a total score equal to 3.305. Finally, the small FPU has a total score equal to 3.964. Therefore, the small FPU has the highest total score after the positive ideal solution.

Then, similar to the WSM method, Equation 6.5 is used to normalize the weights. Next, the model was re-run to calculate the final scores using Equation 6.4 (see Table 6.12).

Table 6.12. Requirements and alternatives final normalized scores for TOPSIS tool

\begin{tabular}{|c|c|c|c|c|c|c|}
\hline \multirow[b]{2}{*}{ Requirements } & \multirow{2}{*}{ 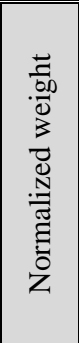 } & \multicolumn{5}{|c|}{$\begin{array}{l}\text { Alternatives ( FPU size) } \\
\text { Normalized scores } \\
\text { Pugh selection matrix: } \\
\text { Feedstock inputs (tpd) }\end{array}$} \\
\hline & & 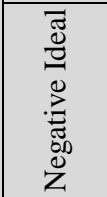 & 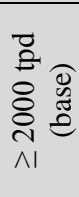 & $\begin{array}{l}\text { D } \\
\text { \& } \\
8 \\
\text { n } \\
1 \\
8 \\
8\end{array}$ & $\begin{array}{l}\text { Z } \\
\vdots \\
0 \\
\text { n } \\
\text { vi }\end{array}$ & 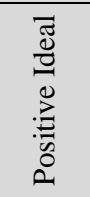 \\
\hline $\mathrm{CO}_{2}$ emission & 0.06 & -0.023 & 0 & 0.006 & -0.023 & 0.006 \\
\hline Resources saving & 0.06 & -0.007 & 0 & 0.007 & -0.007 & 0.007 \\
\hline Design cost & 0.06 & 0 & 0 & 0.017 & 0.040 & 0.040 \\
\hline Capital cost (Including Equipment) & 0.08 & -0.089 & 0 & -0.048 & -0.089 & 0 \\
\hline Equipment cost & 0.06 & -0.073 & 0 & -0.031 & -0.074 & 0 \\
\hline Labor cost & 0.04 & -0.042 & 0 & -0.026 & -0.042 & 0 \\
\hline Production cost in the facility & 0.07 & -0.057 & 0 & -0.028 & -0.057 & 0 \\
\hline Materials cost (Feedstock) & 0.07 & 0 & 0 & 0.025 & 0.045 & 0.045 \\
\hline Transportation cost for feedstock & 0.06 & 0 & 0 & 0.061 & 0.110 & 0.110 \\
\hline Response to market \& policy uncertainties & 0.07 & 0 & 0 & 0.034 & 0.054 & 0.054 \\
\hline Storage cost for feedstock & 0.05 & 0 & 0 & 0.025 & 0.045 & 0.045 \\
\hline Storage cost for produced biofuel & 0.05 & -0.016 & 0 & -0.010 & -0.016 & 0 \\
\hline Annual investments (Maintenance) & 0.07 & 0 & 0 & 0.018 & 0.006 & 0.018 \\
\hline Energy saving & 0.06 & -0.036 & 0 & -0.026 & -0.036 & 0 \\
\hline Biomass availability (Now) & 0.07 & 0 & 0 & 0.047 & 0.082 & 0.082 \\
\hline Operation efficiency & 0.06 & -0.034 & 0 & -0.028 & -0.034 & 0 \\
\hline Policy \& regulations & 0.07 & -0.008 & 0 & -0.008 & 0.015 & 0.015 \\
\hline Number of jobs offered & 0.05 & 0 & 0 & 0.042 & 0.072 & 0.072 \\
\hline Final Scores: & 1 & -0.384 & 0 & 0.078 & 0.093 & 0.495 \\
\hline
\end{tabular}

After the normalization, the positive ideal solution has a total score equal to 0.495 . The negative ideal solution has a total score equal to -0.384 . The large FPU has a total score equal to zero. The medium FPU has a total score equal to 0.078. Finally, the small FPU has a total score 
equal to 0.093 . From Table 6.12 we can conclude that, theoretically, the best option, which meets all stakeholders requirements, is the positive ideal solution which is better than the large FPU by 49.5\%; better than the medium FPU by $41.7 \%$; and better than the small FPU by $40.2 \%$. In addition, the difference between the medium and small FPUs is equal to $1.5 \%$.

After that, the distance $\left(\mathrm{D}_{\mathrm{ij}+}\right)$ between each target alternative (i) and the positive ideal solution $\mathrm{j}_{+}$was calculated using Equation 6.6 (see Table 6.13).

$$
\mathrm{D}_{\mathrm{ij}+}=\sqrt{\sum_{i=1}^{3}\left(V_{j+}-V_{i j}\right)^{2}}
$$

Table 6.13. Distance $\left(\mathrm{D}_{\mathrm{ij}+}\right)$ between alternatives and the positive ideal solution

\begin{tabular}{||l|r|r|r|r|r|r||}
\hline \multirow{4}{*}{ Requirements } & \multicolumn{3}{|c|}{ The distance $\left(\mathrm{D}_{\mathrm{ij}+}\right)$ between the target } \\
alternative $\mathrm{i}$ and the positive ideal $(\mathrm{j}+)$
\end{tabular}


Then, the distance $\left(\mathrm{D}_{\mathrm{ij}-}\right)$ between each target alternative (i) and the negative ideal $\left(\mathrm{j}_{-}\right)$is calculated using Equation 6.7 (see Table 6.14).

$$
\mathrm{D}_{\mathrm{ij}-}=\sqrt{\sum_{i=1}^{3}\left(V_{j-}-V_{i j}\right)^{2}}
$$

Table 6.14. Distance $\left(\mathrm{D}_{\mathrm{ij}}\right)$ between alternatives and the negative ideal solution

\begin{tabular}{|c|c|c|c|c|c|c|}
\hline \multirow{3}{*}{ Requirements } & \multicolumn{6}{|c|}{$\begin{array}{l}\text { The distance }\left(\mathrm{D}_{\mathrm{ij}}\right) \text { between the target } \\
\text { alternative } \mathrm{i} \text { and the negative ideal }(\mathrm{j}-)\end{array}$} \\
\hline & \multicolumn{2}{|c|}{$\geq 2000 \mathrm{tpd}$} & \multicolumn{2}{|c|}{$200-500$ tpd } & \multicolumn{2}{|c|}{$\leq 50$ tpd } \\
\hline & $\begin{array}{l}:= \\
> \\
1 \\
\dot{1} \\
>\end{array}$ & 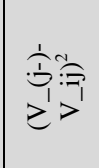 & $\begin{array}{l}:=7 \\
> \\
1 \\
\frac{1}{3} \\
>\end{array}$ & $\begin{array}{l}\frac{1}{3}:= \\
z_{1}>\end{array}$ & $\begin{array}{l}:=7 \\
> \\
1 \\
\frac{1}{3} \\
>\end{array}$ & $\begin{array}{l}\frac{1}{5}= \\
e^{3}=\end{array}$ \\
\hline $\mathrm{CO}_{2}$ emission & -0.023 & 0.001 & -0.029 & 0.001 & 0.000 & 0.000 \\
\hline Resources saving & -0.007 & 0.000 & -0.014 & 0.000 & 0.000 & 0.000 \\
\hline Design cost & 0.000 & 0.000 & -0.017 & 0.000 & -0.040 & 0.002 \\
\hline Capital cost (Including Equipment) & -0.089 & 0.008 & -0.041 & 0.002 & 0.000 & 0.000 \\
\hline Equipment cost & -0.073 & 0.005 & -0.043 & 0.002 & 0.000 & 0.000 \\
\hline Labor cost & -0.042 & 0.002 & -0.015 & 0.000 & 0.000 & 0.000 \\
\hline Production cost in the facility & -0.057 & 0.003 & -0.028 & 0.001 & 0.000 & 0.000 \\
\hline Materials cost (Feedstock) & 0.000 & 0.000 & -0.025 & 0.001 & -0.045 & 0.002 \\
\hline Transportation cost for feedstock & 0.000 & 0.000 & -0.061 & 0.004 & -0.110 & 0.012 \\
\hline Response to market \& policy uncertainties & 0.000 & 0.000 & -0.034 & 0.001 & -0.054 & 0.003 \\
\hline Storage cost for feedstock & 0.000 & 0.000 & -0.025 & 0.001 & -0.045 & 0.002 \\
\hline Storage cost for produced biofuel & -0.016 & 0.000 & -0.005 & 0.000 & 0.000 & 0.000 \\
\hline Annual investments (Maintenance) & 0.000 & 0.000 & -0.018 & 0.000 & -0.006 & 0.000 \\
\hline Energy saving & -0.036 & 0.001 & -0.010 & 0.000 & 0.000 & 0.000 \\
\hline Biomass availability (Now) & 0.000 & 0.000 & -0.047 & 0.002 & -0.082 & 0.007 \\
\hline Operation efficiency & -0.034 & 0.001 & -0.006 & 0.000 & 0.000 & 0.000 \\
\hline Policy \& regulations & -0.008 & 0.000 & 0.000 & 0.000 & -0.023 & 0.001 \\
\hline Number of jobs offered & 0.000 & 0.000 & -0.042 & 0.002 & -0.072 & 0.005 \\
\hline $\begin{array}{l}\left(\left(\mathrm{V}_{\mathrm{j}-}\right)-\left(\mathrm{V}_{\mathrm{ij}}\right)\right)^{2} \\
\end{array}$ & \multicolumn{2}{|c|}{0.021553} & \multicolumn{2}{|c|}{0.016539} & \multicolumn{2}{|c|}{0.033224} \\
\hline $\mathrm{D}_{\mathrm{ij}-=}=\left[\sum\left(\left(\mathrm{V}_{\mathrm{j}-}\right)-\left(\mathrm{V}_{\mathrm{ijj}}\right)\right)^{2}\right]^{1 / 2}$ & \multicolumn{2}{|c|}{0.1468} & \multicolumn{2}{|c|}{0.1286} & \multicolumn{2}{|c|}{0.1823} \\
\hline
\end{tabular}

Finally, the relative closeness $\left(\mathrm{C}_{\mathrm{ij}+}\right)$ between each target alternative (i) and the positive ideal solution $\mathrm{j}_{+}$is calculated using Equation 6.8, and the FPU with the highest $\mathrm{C}_{\mathrm{ij}+}$ score is selected as the best alternative (see Table 6.15). 


$$
C_{i j+}=\frac{D_{i j-}}{\left(D_{i j+}+D_{i j-}\right)}
$$

Where

$$
\begin{aligned}
& C_{i j+}=1 \text { if and only if the alternative (i) has the best condition; and } D_{i j+}=0 \\
& C_{i j+}=0 \text { if and only if the alternative (i) has the worst condition; and } D_{i j+}=1
\end{aligned}
$$

Table 6.15. Relative closeness values to the positive ideal solution

\begin{tabular}{|c|c|}
\hline FPU size & $\mathrm{C}_{\mathrm{ij}+}=$ \\
\hline$\geq 2000 \mathrm{tpd}$ & 0.44578059 \\
\hline $200-500 \mathrm{tpd}$ & 0.529525153 \\
\hline$\leq 50 \mathrm{tpd}$ & 0.550718634 \\
\hline
\end{tabular}

Table 6.15 shows that the $\left(\mathrm{C}_{\mathrm{ij}+}\right)$ for the large FPU is equal to 0.4458 ; for the medium FPU it is equal to 0.5295; and for the small FPU it is equal to 0.5507. The small FPU has the closest value to the positive ideal solution. Therefore, a small FPU is recommended as the best alternative, using TOPSIS, which represents the nearest fit to the stakeholders' requirements.

The last task in this research was to compare and discuss the results from WSM, TOPSIS, with the proposed integrated approach from prior work that solved the same problem (Fawzy, Componation, \& $\mathrm{Hu}, 2015)$. In the prior work, this decision problem was solved using an approach that integrates Pugh Concept Selection Matrix, WSM, and sensitivity analysis using Logical Decision® software. As a result for that work, the small FPU was recommended as the best alternative under limited conditions. From other work on a higher level analysis, the small FPU was recommended as the best alternative from all perspectives except the environmental perspective; from the environmental perspective, the medium FPU was recommended as the best alternative (Fawzy \& Componation, 2015a).

By comparing results from all three integrated approaches, we found that small FPU is recommended as the best alternative. All the approaches ranked the alternatives and could by 
applied at different levels of analysis. On the other hand, each approach provides different insight into the problem. Table 6.16 summarizes all the similarities and differences between the implemented approaches; Table 6.16 also demonstrates the advantages and disadvantages of each approach from our point of view.

Table 6. 16. Used DM Approaches Comparison, Advantages, and Disadvantages from the Researchers' Perspective

\begin{tabular}{|c|c|c|c|c|}
\hline & & \multicolumn{3}{|c|}{ DM tool / approach } \\
\hline & & Pugh Concept + WSM & Pugh Concept + TOPSIS & $\begin{array}{c}\text { Pugh Concept }+ \text { WSM + sensitivity analysis } \\
\text { using Logical Decision software }\end{array}$ \\
\hline \multirow[b]{2}{*}{ 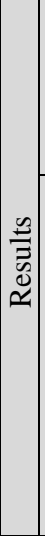 } & & \multicolumn{3}{|l|}{$\begin{array}{l}- \\
- \\
- \\
-\end{array}$} \\
\hline & 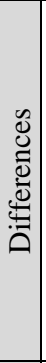 & $\begin{array}{l}\text { - Shows that smallest FPU is better than } \\
\text { the largest unit by } 9.3 \% \\
\text { - Shows that the medium unite it better } \\
\text { than largest unit by } 7.8 \%\end{array}$ & $\begin{array}{l}\text { - Shows that positive ideal FPU } \\
\text { is better than the largest unit by } \\
49.5 \% \\
\text { - Shows that negative ideal FPU } \\
\text { is worse than the largest unit by } \\
38.4 \%\end{array}$ & $\begin{array}{l}\text { - Recommends the smallest FPU as the } \\
\text { best alternative, but under specific } \\
\text { conditions at the requirements-level } \\
\text { analysis (Fawzy, Componation, \& } \\
\text { Hu, 2015) } \\
\text { - Recommends the medium FPU as the } \\
\text { second best alternative based on the } \\
\text { perspectives analysis (Fawzy \& } \\
\text { Componation, 2015a) }\end{array}$ \\
\hline & 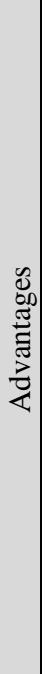 & $\begin{array}{l}\text { - Easy and straightforward process } \\
\text { - Need short time } \\
\text { - Easy to explain to decision makers } \\
\text { - Good for easy decision problems and } \\
\text { some complex ones. }\end{array}$ & $\begin{array}{l}\text { - Illustrate the positive and } \\
\text { negative ideal solution } \\
\text { characteristics } \\
\text { - Shows the problem importance } \\
\text { to the decision makers, which } \\
\text { may be because of the process } \\
\text { complexity } \\
\text { - Shows the distance between } \\
\text { alternatives and their closeness } \\
\text { to the positive ideal solution } \\
\text { Help decision makers to discuss } \\
\text { the possible alternatives to } \\
\text { some extend }\end{array}$ & $\begin{array}{l}\text { - Easy to use } \\
\text { - Easy to explain to decision makers } \\
\text { - Shows the result robustness } \\
\text { - Deep sensitivity analysis } \\
\text { - Easy to modify the data of the } \\
\text { weighting method } \\
\text { - Show the closeness between } \\
\text { alternatives by comparing utilities } \\
\text { values } \\
\text { - Shows the advantages and } \\
\text { disadvantages of each alternative } \\
\text { compare to others } \\
\text { - Help decision makers to discuss the } \\
\text { possible alternatives in depth } \\
\text { - Looks like an integration between } \\
\text { Pugh Concept and Logical Decision for } \\
\text { both results and sensitivity analyses }\end{array}$ \\
\hline
\end{tabular}




\begin{tabular}{|c|c|c|c|}
\hline 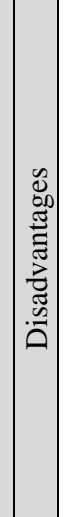 & $\begin{array}{l}\text { - Sensitivity analysis is not a part of the } \\
\text { process, but could be integrated } \\
\text { - Cannot exam the result robustness } \\
\text { - WSM cannot be used without } \\
\text { integration with other tool for } \\
\text { unstructured problems } \\
\text { - Looks like a part of the other two } \\
\text { approaches and need more analysis for } \\
\text { such a complex problem }\end{array}$ & \begin{tabular}{|l} 
- Very long process \\
- Need a lot of time \\
- Have a lot of calculation which \\
require more concentration than \\
other two approaches \\
- Sensitivity analysis is not a part \\
of the process, but could be \\
integrated \\
- Complicated and hard to \\
explain to decision makers \\
- TOPSIS cannot be used without \\
integration with other tool for \\
unstructured problems
\end{tabular} & $\begin{array}{l}\text { - First part could be accomplish using } \\
\text { MS Excel easily, but the sensitivity } \\
\text { analysis is longer and harder by using } \\
\text { MS Excel }\end{array}$ \\
\hline
\end{tabular}

In summary, this research was designed to accomplish six tasks. Out of the first task we determined that each DM tool has its advantages and disadvantages, and selecting the right tool to solve a particular problem is important. In some situations researchers selected multiple tools to provide additional insights about a problem. Therefore, the use of different tools helps better understand the complex problem, especially at this conceptual stage.

The results for the first task indicated that some common DM tools, such as the Pugh Concept Selection Matrix, SMART, and TOPSIS, had been infrequently applied to the renewable energy and biofuel manufacturing industry. On the other hand, WSM, AHP, and ELECTRE are applied in this field individually and are integrated with other tools. In this research we decided to use WSM with the integration of Pugh Concept Selection Matrix as another approach to solve the problem.

The second task summarized previous findings of stakeholders' identification and assessment of perspectives and requirements for biofuel production (Fawzy \& Componation, 2014; Fawzy \& Componation, 2015a; Fawzy, Componation, \& Hu, 2015). The results for the third task were the requirements weights and FPUs sizes evaluation using the Pugh Concept Selection matrix. Finally, we compared the results and highlighted the advantages and 
disadvantages of the approaches used in this work. From this comparison we found that all the used tools showed that the small FPU is the recommended.

\section{Conclusion}

By solving the case study problem using both WSM and TOPSIS tools with the integration of Pugh Matrix (fourth and fifth tasks) we found that the small FPU was recommended as the best option. This result was compatible with a result from a prior work that in which a proposed integrated approach, which integrates the Pugh Concept Selection Matrix, WSM and sensitivity analysis using Logical Decision ${ }^{\circledR}$ software (version 7.2) was applied to the same problem (Fawzy, Componation, \& Hu, 2015).

We concluded that all different approaches used lead to the same recommendation. The integrated approaches explained the recommended FPU size as well as the criteria limitations for this decision. The research addressed the advantages of using sensitivity analysis to explain the robustness of a recommendation, which had been accomplished as a part of the proposed approach in prior work using Logical Decision ${ }^{\circledR}$ software (version 7.2).

To our knowledge, there is no evidence in the existing literature that an investigation of the optimal design specifications in biofuel production has been performed. Therefore, this research introduces the application of the Pugh Concept Selection Matrix and TOPSIS as a significant contribution. A major contribution of the analysis performed in this paper came from the TOPSIS results, in which the characteristics of the theoretical optimal design for FPU manufacturing is identified by the positive ideal solution. In addition, this research identified the negative ideal solution that presented the worst characteristics that FPU designers could avoid in the design stage. 
In conclusion, it was observed that each DM tools has its limitations, but the integration of tools has the benefit of complementing each other, since each DM tool has strengths and weaknesses. We believe that this problem cannot be solved without the integration of at least two DM tools, due to the uncertainty surrounding the decision problem and the lack of available information.

Pugh Concept Selection Matrix seems to be a good tool for qualitative data collection, especially for new complex problems. It follows a straightforward process and it is easy to explain to decision makers. Its integration with WSM or TOPSIS created good approaches to solve this type of problem as an initial investigation. However, it is not a strong tool to provide a final decision for a complex problem without the integration with other tools.

WSM appears to be easy to use, and to explain to decision makers, and it is not timeconsuming. In addition, it seems to be very good for easy decision problems, as well as some complex problems. On the other hand, WSM does not examine the result robustness. Although sensitivity analysis is not a part of the process of WSM, it could be integrated with WSM. In other words, we believe that WSM cannot be used without integration with other tool(s) to solve unstructured complex problems. First four steps of both TOPSIS and the proposed integrated approach, till scores calculation, are the identical steps of WSM method. Using either WSM or TOPSIS alone is not sufficient for this complex problem. Even with having the recommended FPU, more analysis is needs to understand the problem insights and the recommendation dimensions.

Due to the process complexity of TOPSIS, this tool can give decision makers the impression that the decision problem is important. It illustrates the positive and negative ideal solutions characteristics. It also shows the distance between alternatives and their closeness to 
the positive ideal solution that help decision makers to discuss the possible alternatives. Nevertheless, this tool requires a very long process that is time-consuming due to the numerous calculations involved. Also, it appears more complicated than WSM and is harder to explain to decision makers. Similar to WSM, sensitivity analysis is not inherently part of the process, but it could be integrated with it. Furthermore, TOPSIS cannot be used without the integration with other tool(s), such as Pugh Matrix, for solving unstructured problems.

The proposed integrated approach, which integrates Pugh Concept Selection Matrix, WSM, and sensitivity analysis using Logical Decision ${ }^{\circledR}$ software, also has its advantages and disadvantages. Similar to WSM, the first part of this approach is easy to use and explain to decision makers. Moreover, it is easy to modify data and use different weighting methods. This approach shows the result robustness with deep sensitivity analysis. In addition, it illustrates the closeness between alternatives by comparing utility values. In addition, this approach shows the advantages and disadvantages of each alternative in comparison to other alternatives. This approach helps decision makers to discuss the possible alternatives in depth. Overall, it seems that the integration between Pugh Matrix, WSM, and Logical Decision® software for results followed by sensitivity analysis enables good DM under lack of information and uncertainty conditions. In contrast to the first part of this approach that could be accomplished using MS Excel easily, the sensitivity analysis would benefit from the application of dedicated-purpose decision analysis software.

Although a lack of quantitative data may affect the accuracy of results at this conceptual stage of biofuel units manufacturing, the qualitative data collected from SMEs in the field with different backgrounds is sufficient to represent the five BPS perspectives. However, we found that collecting data using interviews is labor-intensive. In addition, using interviews may limit 
the size of collected data, yet it gave us the ability to have more in-depth information and better communication for data collection, which is helpful for the conceptual and theoretical decision phase.

In the future, instead of the SME evaluation using Pugh Matrix for comparative purposes, other weighting methods could be used to solve the problem utilizing the proposed approach. Future work could also involve employing other DM tools in this problem or similar decision problems in the biofuel manufacturing industry to examine their application. In addition, future work could test the FPU size decision by involving wider stakeholder inputs. The proposed approach, like other used approaches, could be applied to different levels of interest, i.e. perspectives or requirements.

As a venue for future work, a web-based system could be developed for the proposed integrated approach to support DM processes for solving decision problems in renewable energy industry. This web-based decision support system could also evaluate future unit sizes for biofuel manufacturing with updated information.

This research evaluated three FPUs using different DM tools to select the best biofuel production strategy that meets stakeholders' requirements. The most appropriate strategy is found to be building multiple small FPUs, which consumes at most 50 tpd of biomass. The use of integrated sets of DM tools developed better understanding of the different available alternatives. This research extended the application of the Pugh Matrix and TOPSIS in the renewable energy field. 


\section{CHAPTER 7. GENERAL CONCLUSIONS}

In conclusion, this dissertation accomplished four tasks: (1) identify biofuel production's stakeholders, (2) identify their perspectives and requirements, (3) assess the biofuel production system to recommend the best FPU based on the stakeholders needs, and (4) investigate the application of different DM tools to solve the problem. The first three tasks represent the phases of a proposed framework to develop a system-oriented approach for the manufacturing, placement, and right sizing of a FPUs manufacturing infrastructure. The fourth task aims to investigate different DM approaches to recommend the best FPU. Managerial insights have been derived based on different utilized DM tools.

This dissertation addresses the following research questions: (1) What are the most common applied DM approaches in this field, (2) What are the evaluation criteria (perspectives \& requirements) for this industry, (3) Can a better understanding of the solution space for the biofuel production problem be gained by using multiple decision-making approaches, and (4) What is the most appropriate biofuel production strategy based on our current understanding of stakeholders' needs.

Given the numerous factors that need to be taken into account, this decision problem is classified as a complex DM problem. This decision problem has a diverse group of BPS involved in the biofuel DM process; each group has a specific objective, perspective, and a list of requirements. Complexity usually increases when there are multiple stakeholders involved in the decision (Grunig \& Kuhn, 2013). In addition, three different alternatives are evaluated 
for their best fit of the BPS needs, which made the decision makers face many alternatives. Also, decision problems that can be described as "complex" are often considered to be "unstructured" (Simon \& Newell, 1958). Furthermore, due to the novelty of the topic and the lack of information, this problem is characterized by uncertainty. The topic is novel because research to better understand biofuel stakeholders is not well developed and a consensus on stakeholder requirements has not been reached. The uncertainty is caused by the lack of consensus and the immaturity of the biofuel technologies. Therefore, this problem is classified as an uncertain unstructured complex problem.

The biofuel production system assessment and the best FPU recommendation accomplished first by proposing an integrated DM approach that incorporates Pugh Matrix, WSM, and sensitivity analysis using Logical Decision ${ }^{\circledR}$ software. Then, WSM and TOPSIS were integrated with Pugh Matrix as two other approaches to assess the biofuel production system.

This study started by proposing a system-oriented approach to be accomplished through six consecutive phases. These phases are:

- Identification of biofuel production's stakeholder.

- Identification of their perspectives and requirements.

- Assessment of biofuel production systems.

- Application of process controls strategies.

- Application of advanced manufacturing technologies.

- Modeling and simulation of the biofuel production facilities. 
This dissertation aims to investigate the first three phases in four research studies as detailed in chapters 2 through 6 . The other three phases, phases 4 through 6 , are reserved as future research directions.

As an outcome of the first three phases mentioned above, 36 groups of BPS are identified as decision makers. A total of 31 unique requirements were identified from the groups, and a subset of 18 requirements was selected for this study based on their frequency in previous research in the field. The 18 requirements were then grouped into 5 different perspectives, including economic, environmental, technical, social, and legal. Economic criteria are generated, namely, the capital cost, equipment cost, production cost, design cost, labor cost, materials cost, transportation cost for feedstock, unit flexibility (response to market \& policy uncertainties), annual investments (maintenance), storage cost for feedstock, and storage cost for produced biofuel. Environmental criteria include the $\mathrm{CO} 2$ emission and resources saving. For technology, criteria are generated to reflect energy saving, biomass availability, and operation efficiency. Social requirement represented the number of jobs offered in this facility. Finally, the legal criterion reflects policy \& regulations. It is necessary to reach a balance between all perspectives and requirements in appropriate ways while making the decision.

There are two novel and significant contributions from this research. First is the use of a comprehensive set of BPS requirements and perspectives to study biofuel production. Second is the use of an integrated set of DM tools to develop a better understanding of the different biofuel production solutions that are currently available.

While there is a significant body of work on technological advances to improve production of biofuel, only limited work has been completed on understanding stakeholders. 
This research found that most prior work only looked at individual or a small set of requirements. The comprehensive literature review and work with SMEs allowed the development of a broader understanding of the different and often conflicting requirements. The grouping of similar requirements into perspectives helped clarify some of the issues related to biofuel production, in particular the tradeoffs between the economic and environmental perspectives. Often biofuel production approaches that are desirable from one perspective are less so from other perspectives. This is also a challenge because of the limited data available to understand how these requirements are met. The challenge is compounded by the mix of quantitative and qualitative data used to measure performance of biofuel production against these requirements.

One significant advantage in the research approach used was the inclusion of a team of SMEs to review and assess the requirements list. Published research tends to lag state of the art in any field. SMEs were able to clarify and at times point out future trends that published research had not fully identified. SMEs were also a critical part of the assessment process. They validated the research procedures and validated the data collection instrument.

A limited set of DM tools has been used in renewable energy research. DM can become a powerful tool to analyze sustainable energy system (Wang, Jing, Zhang, \& Zhao, 2009). This research identified what DM tools have been used in renewable energy, and then identified what the advantages and disadvantages of each approach are. Some common DM tools, such as the Pugh Concept Selection Matrix, SMART, and TOPSIS, had not been used widely in the renewable energy and biofuel industries. WSM, AHP, and ELECTRE have been widely applied in this field, individually and with integrations with other tools. To gain a better 
understanding of biofuel production this research used a set DM tools individually and paired together.

$\mathrm{DM}$ in the early stages of a product's life cycle is difficult because there is limited data. This research showed that the Pugh Concept Selection Matrix was an effective approach to deal with the complexity and uncertainty commonly found early in product development, in particular in the conceptual design phase. The use of the WSM was also found to be effective and in particular when combined with a sensitivity analysis. The sensitivity analysis helped illustrate how changes in BPS requirement priorities could affect the selection of a recommended biofuel production approach. The different recommendations that were presented when economic or environmental requirements were prioritized helped illustrate the dissuasions often seen in the media and from prior research.

Further work using the Pugh Concept Selection Matrix in combination with WSM and then with TOPSIS helped clarify the solution space by identifying biofuel production strategies that were most effective at meeting the majority of BPS requirements and perspectives. TOPSIS in particular helped identify how far current solutions are from a theatrical optimal solution.

The methods presented in this work were found to be effective in structuring a complex decision and appear to be easily adaptable to a range of similar problems. Of particular value were the use of a team of SMEs in combination with a rigorous review of prior work, and the use of multiple DM tools to provide a better understanding of the problem solution space.

The specific problem studied in this effort was the assessment of alternative biofuel production approaches. Specifically, what FPU size should be recommended to meet BPS requirements. The FPUs selected, 2,000 tpd, 200 to 500 tpd, and 50 tpd, represented the most 
commonly produced units in the market currently. The application of the DM tools individually and in combination showed that in the majority of cases the small FPU, 50 tpd, was the most effective in meeting the majority of BPS requirements. Significant variances in requirement priorities could change the recommendation. The changes would need to be significant. The importance of understanding all stakeholder requirements and selecting a DM tool that was applicable to the problem had been found to be an important step.

It was noticed that each DM tool had its limitations, but the integration of tools had the benefit of complementing each other since each DM tool has its strength and weaknesses. This problem is easier to solve with the integration of at least two DM tools.

The data collection done through interviewing a group of SMEs in the field. It was extremely difficult to conduct these interviews in terms of time and effort. Scheduling the interviews was the most difficult part in the data collection, as the most experienced people in this area have a lot of duties and responsibilities due to their position. In addition, they usually have a lot of activities and frequent traveling schedules which made the interviews not an easy task for the interviewer.

Researchers usually look to have a better design, but no one, up to our knowledge, ever tried to investigate the optimal design specifications in biofuel production. On contrast, as a part of TOPSIS results, this research proposed the characteristics of the theoretical optimal design for FPUs manufacturing by identifying the positive ideal solution. In addition, this research identified the negative ideal solution that presented the worst characteristics those FPUs designers could avoid in the design stage.

Even the lack of quantitative data may affect the result accuracy at this conceptual stage of biofuel units manufacturing, yet the qualitative data collected from SMEs in the field with 
different backgrounds is sufficient to represent the five BPS perspectives. It is noticed that, collecting data using interviews is a labor intensive process. In addition, using interviews may have limited the size of collected data, yet it gave us the ability to have an in depth understanding of the problem and allowed better communication for data collection, which is helpful for conceptual and theoretical decisions phase.

In the future, other weighting method could be used to solve the problem using the proposed approach instead of the SMEs evaluation using Pugh Concept Selection Matrix for comparative purposes. Future work could also involve employing other DM tools in this problem or similar decision problems in the biofuel manufacturing industry to examine their application. In addition, future work could test the FPU size decision by involving wider stakeholder inputs or including the most important potential requirements recommended by SMEs. The proposed approach, like other approaches, could be applied on different levels of interest i.e. perspectives or requirements.

As a venue for future work, a web-based system could be developed for the proposed integrated approach to support DM processes for solving decision problems in renewable energy industry. This system could also evaluate future unit sizes for biofuel manufacturing with updated information. 


\section{REFERENCES}

Al-Amre, Ahmad S. and Al-Fowzan, Naser M. (19/4/1421 A.H. - 8/6/1998) Employees Resistance to Change in governmental sectors, its reasons and ways of administration (Fieldwork presented in a scientific conference), Jeddah: King Saud University, Science Faculty, General Administration Department, Saudi Arabia

An, H., Wilhelm, W. E., \& Searcy, S. W. (2011). Biofuel and petroleum-based fuel supply chain research: a literature review. Biomass \& Bioenergy, 35(9), 3763-3774.

Anex, R. P., Aden, A., Kazi, F. K., Fortman, J., Swanson, R. M., Wright, M. M., . . Dutta, A. (2010). Techno-economic comparison of biomass-to-transportation fuels via pyrolysis, gasification, and biochemical pathways. Fuel, 89, pp. 29-35.

Anonymous, Change Management Toolbook, (2013). Retrieved from: http://dev.changemanagement-toolbook.com/mod/book/print. php?id=74\&chapterid=68.

Arrow, K. J. (1962). The Economic Implications of Learning by Doing. The Review of Economic Studies, 29(3), pp. 155-173.

Awudu, I., \& Zhang, J. (2012). Uncertainties and Sustainability Concepts in Biofuel Supply Chain Management: A Review. Renewable and Sustainable Energy Reviews, 16(2), pp. 1359-1368.

Ayoub, N., Martins, R., Wang, K., Seki, H., \& Naka, Y. (2007). Two levels decision system for efficient planning and implementation of bioenergy production. Energy conversion and management, 48(3), 709-723.

Balat, M., \& Balat, H. (2009). Recent trends in global production and utilization of bio-ethanol fuel. Applied energy, 86(11), 2273- 2282.

Bardi, U. (2009). Peak oil: The four staFges of a new idea. Energy, 34(3), 323-326.

Beccali, M., Cellura, M., \& Mistretta, M. (2003). Decision-making in energy planning: application of the ELECTRE method at regional level for the diffusion of renewable energy technology. Renewable Energy, 28, 2063-2087.

Belton, V., \& Stewart, T. (2002). Multiple Criteria Decision Analysis: An Integrated Approach. Norwell, Massachusetts: Kluwer Academic Publishers.

Biggam, J. (2011). Succeeding With Your Master'S Dissertation: A Step-By-Step Handbook. New York: McGraw-Hill International.

Bomb, C., McCormick, K., Deurwaarder, E., \& Kåberger, T. (2007). Biofuels for transport in Europe: Lessons from Germany and the UK. Energy Policy, 35(4), 2256-2267. 
Bridgwater, A., \& Peacocke, G. (2000). Fast pyrolysis processes for biomass. Renewable and Sustainable Energy Reviews, 1-73.

Browne, D., O'Regan, B., \& Moles, R. (2010). Use of multi-criteria decision analysis to explore alternative domestic energy and electricity policy scenarios in an Irish city-region. Energy, 35(2), PP. 518-528.

Cadre, E., \& Orset, C. (2010). Irreversible investment, Uncertainties, and Ambiguity: the Case of Bioenergy Sector. working papers, Cahiers de Recherche.

Cervone, H. F. (2009a). Applied digital library project management: Using Pugh matrix analysis in complex decision-making situations. OCLC Systems \& Services, 25(4), pp. 228-232.

Cervone, H. F. (2009b). Applied digital library project management: Using paired comparison analysis to determine relative importance. OCLC Systems \& Services, 25(3), pp. 16-26.

Chelst, K., \& Canbolat, Y. (2011). Value-Added Decision Making for Managers, 1. New York: CRC Press.

Cipollini, K. A., Maruyama, A. L., \& Zimmerman, C. L. (2005). Planning for Restoration: A Decision Analysis Approach to Prioritization. Restoration Ecology Journal, 13(3), pp. 460-470.

Coelho, M. C., Dutta, S., \& Silva, F. N. (2013). Biodiesel from microalgae in lagoons: an acceptable alternative fuel to the transportation crunch? Washington, D.C.: The 92nd Annual Meeting of the Transportation Research Board.

Componation, P. J., \& Collopy, P. D. (2012). Systems Engineering Theory: Addressing Complesity and Uncertainty in Space Systems Architecture. AIAA Space 2012 Conference \& Exposition. Pasadena, CA, USA.

Componation, P. J., \& Nicholls, G. M. (2011). Decision Maker Perceptions of Alternative Trade Study Approaches. The Journal of Reliability, Maintainability, and Supportability in Systems Engineering, 5, pp. 5-12.

Componation, P., "The Case for a Structured Analytic Decision Process," [PowerPoint slides] (2013). Retrieved from https://bb.its.iastate.edu/ webapps/portal/frameset.jsp

Componation, P., Dorneich, M., Hu, G., \& Nicholls, G. (2013). Applying Alternative Decisionmaking Approaches to a Complex Supplier Selection Problem. (A. Krishnamurthy, \& W. Chan, Eds.) Proceedings of the 2013 Industrial and Systems Engineering Research Conference.

Cook, W. D., Kress, M., \& Seiford, L. M. (1996). Data Envelopment Analysis in the Presence of both Quantitative and Qualitatie Factors. Journal of Operations Research Society, 47, 945-953. 
Dahlgren, E., Göçmen, C., Lackner, K., \& van Ryzin, G. (2013). Small modular infrastructure. The Engineering Economist, 58(4), pp. 231-264.

Dahlgren, E., Lackner, K. S., Gocmen, C., \& van Ryzin, G. (2012, July). Small Modular Infrastructure. Decision, Risk \& Operations Working Paper Series. Columbia Business School.

Dal-Mas, M., Giarola, G., Zamboni, A., \& Bezzo, F. (2011). Strategic Design and Investment Capacity Planning of the Ethanol Supply Chain under Price Uncertainty. Biomass and Bioenergy, 35(5), 2059-2071.

DCLG. (2009). Department for Communities and Local Governm. Multi-criteria analysis: A manual. London: Crown.

DOE. (2011). A Multifaceted Mathematical Approach for Complex Systems. DOE, WOE Workshop on Mathematics for Complex Systems.

Dunnet, A. J., Adjiman, C. S., \& Shah, N. (2008). A Spatially Explicit Whole-system Modl of the Lignocellulocis Bioethanol Supply Chain: An Assessment of Decentralized Processing Potential. Biotechnology for Biofuels, 1, 1-13.

Dwivedi, P., \& Alavalapati, J. (2009). Stakeholders' perceptions on forest biomass-based bioenergy development in the southern US. Energy Policy, 37(5), pp. 1999-2007.

Dyer, J. (2005). MAUT — Multiattribute Utility Theory. In J. Figueira, S. Greco, \& M. Ehrogott, Multiple Criteria Decision Analysis: State of the Art Surveys, 78, pp. 265-292. New York: Springer New York.

EBTP Stakeholders. ( 18 December, 2012). EBTP Stakeholders. In The European Biofuels Technology Platform . Retrieved March 16, 2013, from http://www.biofuelstp.eu/stakeholders.html.

EIA. (2001). Monthely Energy Review. (The U.S. Energy Information Administration) Retrieved December 03, 2013, from Table 1.3 and 10.1:

http://www.eia.gov/energyexplained/index.cfm?page=renewable_home

EIA. (2002, May). PETROLEUM \& OTHER LIQUIDS. Retrieved from The U.S. Energy Information Administration (EIA):

http://www.eia.gov/pub/oil_gas/petroleum/analysis_publications/c hronology/petroleumchronology2000.htm

EIA. (2013). Monthely Energy Review. (The U.S. Energy Information Administration) Retrieved December 03, 2013, from Table 1.3 and 10.1: http://www.eia.gov/energyexplained/index.cfm?page=renewable_home 
EIA. (2014). Monthely Energy Review. (The U.S. Energy Information Administration) Retrieved December 01, 2014, from Table 1.3 and 10.1: http://www.eia.gov/energyexplained/index.cfm?page=renewable_home

Eksioglu, S. D., Acharya, A., Leightley, L. E., \& Srora, S. (2009). Analyzing the Design and Management of Biomass-to-Biorefinery Supply Chain. Computer and Industrial Engineering, 57(4), 1342- 1352.

Fawzy, M. F., \& Componation, P. J. (2015b). Biofuel Production: Fast Pyrolysis Units' Manufacturing Infrastructure. 2015 International Conference on Industrial Engineering and Operations Management (pp. PP. 2577 - 2583). Dubai, United Arab Emirates (UAE): IEEE Xplore.

Fawzy, M., \& Componation, P. (2014). Biofuel Production: Stakeholders' Identification. Journal of Management and Engineering Integration, 7(1), pp. 15-22.

Fawzy, M., \& Componation, P. (2015a). Biofuel Production: Utilizing Stakeholders' Perspectives. Engineering Management Journal, 27(2), In press.

Fawzy, M., Componation, P., \& Hu, G. (2015). Stakeholders' Requirements Assessment for Biofuel Production. Energy, (In press).

Fortin, E. (2011). Multi-stakeholder initiatives to regulate biofuels: the Roundtable for Sustainable Biofuels.

Freeman, R. E. (1984). Strategic management: A stakeholder approach. Cambridge University Press.

Gagnon, R. J., \& Haldar, S. (1997). Assessing Advanced Engineering Technologies. International Journal of Technology Managment, 14(2/3/4).

Georgia Tech Manufacturing Institute. (2011, January). NSF Energy Manufacturing Workshop January 2011. Retrieved 12 12, 2014, from http://www.manufacturing.gatech.edu/energy/national-science-foundation-energymanufacturing-workshop/january-2011

Georgia Tech Manufacturing Institution. (2009, March 24-25). NSF Energy Manufacturing Workshop - March 2009. Retrieved 12 12, 2014, from http://www.manufacturing.gatech.edu/energy/national-science-foundation-energymanufacturing-workshop/march-2009

Georgopoulou, E., Lalas, D., \& Papagiannakis, L. (1997). A multicriteria decision aid approach for energy planning problems: the case of renewable energy option. European Journal of Operational Research, 103, 38-54.

Gnansounou, E. (2011). Assessing the Sustainability of Biofuels: A Logic-Based Model. Energy, 36, 2089-2096. 
Goodwin, P., \& Wright, G. (2009). Decision Analysis for Management Judgment (4 ed.). Chichester, West Sussex, England: John Wiley and Sons, Ltd.

Gorry, G. A., \& Scott Morton, M. S. (1971). A Framework for Management Information Systems. Sloan Management Review, 13(1), 50-70.

Govindan, K., Rajendran, S., Sarkis, J., \& Murugesan, P. (2013). Multi criteria decision making approaches for green supplier evaluation and selection: A literature review. Journal of Cleaner Production, (In Press), pp. 1-18.

Gregory, R., \& Keeney, R. L. (1994). Creating Policy Alternatives Using Stakeholder Values. Management Science, 40(8), 1035-1048.

Grunig, R., \& Kuhn, R. (2013). Successful Decision-Making. Heidelberg: Springer-Verlag Berlin.

Hamaker, J. W., \& Componation, P. J. (2010). Using our Right Brains to Improve Space Project Cost Estimating. Journal of Cost Analysis and Parametrics, 1(2), pp. 59-75.

Harris, G. A., Componation, P. J., \& Farrington, P. A. (2010). An Exploration of Fisher's Framework for the Alignment of Supply Chain Strategy with Product Characteristics. Engineering Management Journal, 22(4).

Ho, W., Xu, X., \& Dey, P. (2010). Multi-criteria decision making approaches for supplier evaluation and selection: A literature review. European Journal of Operational Research, 202(1), pp. 16-24.

Honoré, P. A., Fos, P. J., Smith, T., Riley, M., \& Kramarz, K. (2010). Decision Science: A Scientific Approach to Enhance Public Health Budgeting. Journal of Public Health Management and Practice, 16(2), pp. 98-103.

Huang, J. P., Poh, K. L., \& Ang, B. W. (1995). Decision analysis in energy and environmental modeling. Energy, 20(9), 843-855.

Huertas, D. A., Berndes, G., Holmén, M., \& Sparovek, G. (2010). Sustainability certification of bioethanol: how is it perceived by Brazilian stakeholders? Biofuels, Bioproducts and Biorefining, 4(4), 369-384.

Jack, M. W. (2009). Scaling laws and technology development strategies for biorefineries and bioenergy plants. Bioresource Technology, 100(24), pp. 6324-6330.

Jones, M., Hope, C., \& Hughes, R. (1990). A multi-attribute value model for the study of UK energy policy. The Journal of the Operational Research Society, 41(10), 919-929.

Jordanian government, Jordanian egovernment program (2007) Change Management Manual, Edition 1, Pp. 2-14, Jordan: Jordanian government. 
Karsak, E. E., \& Ahiska, S. S. (2005). Practical Common Weight Multi-criteria Decision-making Approach with Improved Discriminatatitive Power for Technology Selection. International Journal of Production Research, 46(24), 1537-1554.

Karvetski, C. W., Lambert, J. H., \& Linkov, I. (2010). Scenario and Multiple Criteria Decision Analysis for Energy and Environmental Security of Military and Industrial Installations. Integrated Environmental Assessment and Management, 7(2), pp. 228-236.

Kim, J., \& Realff, M. J. (2011, September). Optimal Design and Global Sensititivity Analysis of Biomass Supply Chain Networks for Biofuels under Uncertainity. Energy Systems Engineering, 35(9), 1738-1751.

Kotha, S., \& Swamidass, P. (2000). Strategy, advanced manufacturing technology and performance: empirical evidence from US manufacturing firms. Journal of Operations Management, 18(3), pp. 257-277.

Kurka, T. (2013). Application of the analytic hierarchy process to evaluate the regional sustainability of bioenergy developments. Energy, 62, PP. 393-402.

LaClaire, C. E., Barrett, C. J., \& Hall, K. (2004). Technical, environmental and economic feasibility of bio-oil in new hampshire's north country. Durham, NH: University of New Hampshire.

Lane, J. (2012, November 20). Little Big Tech: Can FischerTropsch Technology Work at Smaller Scale? Biofuels Digest.

Larasati, A., Liu, T., \& Epplin, F. M. (2012, December). An Analysis of Logistic Costs to Determine Optimal Size of a Biofuel Refinery. Engineering Management Journal, 24(4), pp. 63-72.

Lee, J. S. H., Rist, L., Obidzinski, K., Ghazoul, J., \& Koh, L. P. (2011). No farmer left behind in sustainable biofuel production. Biological Conservation, 144(10), 2512-2516.

Liu, F. H., \& Hai, H. L. (2005). The voting analytic hierarchy process method for selecting supplier. International Journal of Production Economics, 97(3), PP. 308-317.

Loken, E. (2007). Use of multicriteria decision analysis methods for energy planning problems. Renewable \& Sustainable Energy Reviews, 11, 1584-1595.

Markanda, A., \& Pemberton, M. (2010). Energy Security, Energy Modeling and Uncertainties. Energy Policy, 38, 1609-1613.

Mateen, F. J., \& Brook, R. D. (2011). Air pollution as an emerging global risk factor for stroke. JAMA, 305(12), 1240-1241.

McDonald, A., \& Schrattenholzer, L. (2001). Learning Rates for Energy Technologies. Energy Policy, 29(4), pp. 255-261. 
Meiera, H., and Schrödera, S., "Improving changeability by designing modularly structured production systems - considering production systems as sociotechnical systems," Procedia Computer Science, (March 19-22, 2013).

Meyer, S. (2007). US Biofuel Analysis Under Uncertainties and Volatility in the Biofuel Industry: . FAPRI.

Michalopoulos, A., Landeweerd, L., Werf-Kulichova, Z. V., Puylaert, P. G., \& Osseweijer, P. (2011). Contrasts and synergies in different biofuel reports. Interface Focus, 1(2), pp. 248-254.

Min, H. (1994). International supplier selection: A multi-attribute utility approach. International Journal of Physical Distribution \& Logistics Management, 24(5), pp. 24-33.

Mintzberg, H., Raisinghani, D., \& Theoret, A. (1976). The Structure of 'Unstructured' Decision Processes. Administrative Science Quarterly, 21(2), 246-275.

Mitchell, R. K., Agle, B. R., \& Wood, D. J. (1997). Toward a theory of stakeholder identification and salience: Defining the principle of who and what really counts. Academy of management review, 22(4), 853-886.

Moffett, A., Dyer, J. S., \& Sarkar, S. (2006). Integrating biodiversity representation with multiple criteria in North-Central Namibia using non-dominated alternatives and a modified analytic hierarchy process. Biological Conservation, 129(2), pp. 181-191.

Perimenis, A., Walimwipi, H., Zinoviev, S., Muller-Langer, F., \& Miertus, S. (2011). Development of a decision support tool for the assessment of biofuels. Energy Policy, 39(1), 1782-1793.

Pohekar, S. D., \& Ramachandran, M. (2004). Application of Multi-Criteria Decision Making to Sustainable Energy Planning - A Review. Renewable and Sustainable Energy Reviews, 8, 365-381.

Prasad, A. S., \& Somasekhara, N. (1990, September). The Analytical Hierarchy Process for Choice of Technology. Forecasting Social Change, 38(2), 151-158.

Pugh, S. (1991). Total design: Integrated methods for successful product engineering. Wokingham: Addison-Wesley.

Punniyamoorthy, M., \& Ragavan, P. V. (2003). A Strategic Decision Model for the Justification of Technology Selection. International Journal of Advanced Manufacturing Technology, 21, 72-78.

Qin, R., Grasman, S. E., Long, S., Lin, Y., \& Thomas, M. (2012). A Framework of CostEffectiveness Analysis for Alternative Energy Strategies. Engineering Management Journal, 24(4), PP. 18-35. 
Ravindranath, N., Manuvie, H. R., Fargione, J., Canadell, P., Berndes, G., \& Woods, J. (2009). GHG Implications of Land use and Land Conversion to Biofuel Crops. Report, Biofuels.

Rentizelas, A. A., Tolis, A., \& Tatsiopoulos, I. P. (2009). Logistics Issues of Biomass: The Storage Proglem and Multi-biomass Supply Chain. Renewable and Sustainable Energy Reviews, 12, 887-894.

Richard, T. L. (2010). Challenges in scaling up biofuels infrastructure. Science, 329(5993), pp. 793-796.

Ringer, M., Putsche, V., \& Scahill, J. (2006). Large-scale pyrolysis oil production: A technology assessment and economic analysis. Colorado: National Renewable Energy Laboratory.

Saaty, T. (1978). Modeling Unstructured Decision Problems - The Theory of Analytical Hierarchies. Mathematics and Computers in Simulation, 20(3), 147-158.

Schnepf, R., \& Yacobucci, B. D. (2010, July). Renewable Fuel Standard (RFS): Overview and Issues. In CRS Report for Congress (No. R40155).

Scott, J. A., Ho, W., \& Dey, P. K. (2012). A Review of Multi-Criteria Decision-Making Methods for Bioenergy Systems. Energy, 42, 146-156.

Shafiee, S., \& Topal, E. (2009). When will fossil fuel reserves be diminished? Energy Policy, 37(1), pp. 181-189.

Shipp, S. S., Gupta, N., Scott, J. A., Weber, C. L., Finnin, M. S., \& Lal, B. (2012). Advancing Manufacturing to New Frontiers: Increasing Opportunities for Society. Innovations, 7(3), $71-81$

Simon, H. A., \& Newell, A. (1958). Heuristic Problem Solving: The Next Advance in Operations Research. Operations Research, 6(1), 1-10.

Smith, G., \& Speiser, F. (1991). Logical Decision: multi-measure decision analysis software. Golden. CO: PDQ Printing.

Swanson, R. M., Platon, A., Satrio, J. A., \& Brown, R. C. (2010). Techno-economic Analysis of Biomass-to-Liquids Production based on Gasification. Fuel, 89(1), pp. 11-19.

Talamini E, et al., (2012). "The Composition and Impact of Stakeholders' Agendas on U.S. Ethanol Production,” Energy Policy Journal, Vol. 50, No. 1, pp. 647-658.

Theodorou, S., Florides, G., \& Tassou, S. (2010). The use of multiple criteria decision making methodologies for the promotion of RES through funding schemes in Cyprus: a review. Energy Policy, 38, 7783-7792.

Tipping, J. W., \& Zeffren, E. (1995). Assessing the Value of your Technology. Research in Technology Management, 38(5). 
Triantaphyllou, E., \& Baig, K. (2005). The Impact of Aggregating Benefit and Cost Criteria in Four MCDA Methods. IEEE Transactions on Engineering Management, 52(2), 213-226.

Tsuchiya, H., \& Kobayashi, O. (2004). Mass Production Cost of Pem Fuel Cells by Learning Curve. International Journal of Hydrogen Energy, 29(10), pp. 285-900.

Turcksin L., et al., (2010). "A multi-actor multicriteria framework to assess the stakeholder support for different biofuel options: The case of Belgium," Energy Policy Journal, 39(1), pp. 200-214.

Turcksin, L., Macharis, C., (2009), "Dedicated workshop for biofuel representatives" in Belgium on 4th of June 2009, Vrije Universiteit Brussel.

Tzeng, G.-H., Shiau., T.-a., \& Lin, C.-Y. (1992). Application of multicriteria decision making to the evaluation of new energy system development in Taiwan. Energy, 17(10), PP. 983992.

U.S. Department of Energy, Office of Energy Efficiency \& Renewable Energy. (2014). ENERGY.GOV. Retrieved 12 12, 2014, from http://energy.gov/eere/cemi/clean-energymanufacturing-initiative

United States Department of Agriculture, Summary Report of Stakeholder Responses to USDA's Regional Biofuel Roadmap, (2012). Retrieved from: http://dev.changemanagementtoolbook.com $/ \mathrm{mod} /$ book/print.php?id=74\&chapterid=68.

Van der Horst, D., \& Vermeylen, S. (2011). Spatial scale and social impacts of biofuel production. Biomass and Bioenergy, 35(6), 2435- 2443.

Voegele, E. (2012, September 07). The Renewable Energy Components of the Democratic and Republican Platforms. Biomass Magazine.

Wang, J. J., Jing, Y. Y., Zhang, C. F., \& Zhao, J. H. (2009). Review on Multi-Criteria Decision Analysis Aid in Sustainable Energy Decision-Making. Renewable and Sustainable Energy Reviews, 13(9), PP. 2263-2278..

Wang, J. J., Jing, Y. Y., Zhang, C. F., Shi, G. H., \& Zhang, X. T. (2008). A fuzzy multi-criteria decision-making model for trigeneration system. Energy Policy, 36(10), PP. 3823-3832..

Wang, L., "Potential Competition for Biomass between Biopower and Biofuel under RPS and RFS2," [PowerPoint slides] (2013). Retrieved from http://echodelivery.cvm.iastate.edu:8080/ess/eco/pr esentation/85d4095e-b12a-47e8$\underline{\text { b897- } 15 \text { b537feaa25 }}$

Wright, M. M., Brown, R. C., \& Boateng, A. A. (2008). Distributed processing of biomass to bio-oil for subsequent production of Fischer-Tropsch liquids. Biofuels, Bioproducts and Biorefining, 2(3), pp. 229-239. 
Wright, M. M., Daugaard, D. E., Satria, J. A., \& Brown, R. C. (2010). Techno-economic Analysis of Biomass Fast Pyrolysis to Transportation Fuels. Fuel, 89(1), pp. S2-S10.

Wright, M., \& Brown, R. C. (2007). Establishing the optimal sizes of different kinds of biorefineries. Biofuels Bioproducts and Biorefining, 1(3), pp. 191-200.

Yang, H. T., \& Chen, S. L. (1989). Incorporating a multi-criteria decision procedure into the combined dynamic programming/production simulation algorithm for generation expansion planning. IEEE Transactions on Power Systems, 4(1), 165-175.

Yoon, K., \& Hwang, C. (1995). Multiple Attribute Decision Making: An Introduction. Thousand Oaks, California: Sage.

Youngs, H. L. (2012). The Effects of Stakeholder Values on Biofuel Feedstock Choices. In C. Taylor, R. Lomneth, \& F. Wood-Black, Perspectives on Biofuels: Potential Benefits and Possible Pitfalls, 1, pp. 29-67. Washington, DC, USA: American Chemical Society.

Zhou, P., Ang, B. W., \& Poh, K. L. (2006). Decision Analysis in Energy and Environmental Modeling: An Update. Energy, 31, 2604-2622. 
